\title{
Higher Quarkonia
}

\section{T.Barnes, ${ }^{1 *}$ F.E.Close, ${ }^{2}$ P.R.Page ${ }^{3}$ E.S.Swanson ${ }^{4}$}

${ }^{1}$ Theoretical and Computational Physics Section, Oak Ridge National Laboratory, Oak Ridge, TN 37831-6373, USA

Department of Physics and Astronomy, University of Tennessee, Knoxville, TN 37996-1501, USA

${ }^{2}$ Particle Theory, Rutherford-Appleton Laboratory, Chilton, Didcot OX11 OQX, UK

${ }^{3}$ Department of Physics and Astronomy, University of Manchester, Manchester M13 9PL, UK

${ }^{4}$ Department of Physics, North Carolina State University, Raleigh, NC 27695-8202, USA

(September 1996)

\begin{abstract}
We discriminate gluonic hadrons from conventional $q \bar{q}$ states by surveying radial and orbital excitations of all $\mathrm{I}=0$ and $\mathrm{I}=1 n \bar{n}$ systems anticipated up to $2.1 \mathrm{GeV}$. We give detailed predictions of their quasi-two-body branching fractions and identify characteristic decay modes that can isolate quarkonia. Several of the "missing mesons" with $\mathrm{L}_{q \bar{q}}=2$ and $\mathrm{L}_{q \bar{q}}=3$ are predicted to decay dominantly into certain $\mathrm{S}+\mathrm{P}$ and $\mathrm{S}+\mathrm{D}$ modes, and should appear in experimental searches for hybrids in the same mass region. We also consider the topical issues of whether some of the recently discovered or controversial meson resonances, including glueball and hybrid candidates, can be accommodated as quarkonia.
\end{abstract}

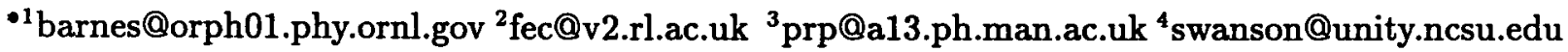




\section{INTRODUCTION.}

Theoretical studies of light hadron spectroscopy have led to the widespread belief that gluonic excitations are present in the spectrum of hadrons, so more resonances should be observed than are predicted by the conventional $q \bar{q}$ and $q q q$ quark model. The two general categories of gluonic mesons expected are glueballs (dominated by pure glue basis states) and hybrids (dominated by basis states in which a $q \bar{q}$ is combined with a gluonic excitation).

Some of these novel states, notably the light hybrids, are predicted to have exotic quantum numbers (forbidden to $q \bar{q}$ ), such as $\mathrm{J}^{P C}=1^{-+}$. The confirmation of such a resonance would be proof of the existence of exotic non- $q \bar{q}$ states, and would be a crucial step towards establishing the spectrum of gluonic states. There are detailed theoretical predictions for the decays of these exotic hybrids [1,2], which have motivated several experimental studies of purportedly favored hybrid channels such as $b_{1} \pi$ and $f_{1} \pi$.

Although one would prefer to find these unambiguously non- $q \bar{q} \mathrm{~J}^{P C}$-exotics, glueballs and hybrids with non-exotic quantum numbers are also expected. For example, in the flux tube model the lowest hybrid multiplet, expected at $\approx 1.8-1.9 \mathrm{GeV}[3,4]$, contains the non-exotics $\mathrm{J}^{P C}=0^{-+}, 1^{ \pm \pm}, 1^{+-}$and $2^{-+}$in addition to the exotics $0^{+-}, 1^{-+}$and $2^{+-}$. To identify these non-exotic states one needs to distinguish them from the "background" of radial and orbital $q \bar{q}$ excitations in the mass region $\approx 1.5-2.5 \mathrm{GeV}$, where the first few gluonic levels are anticipated $[5,6]$.

Our point of departure is to calculate the two-body decay modes of all radial and orbital excitations of $n \bar{n}$ states $(n=u, d)$ anticipated up to $2.1 \mathrm{GeV}$. This includes $2 \mathrm{~S}$, $3 \mathrm{~S}, 2 \mathrm{P}, 1 \mathrm{D}$ and $1 \mathrm{~F}$ multiplets, a total of 32 resonances in the $n \bar{n}$ sector. We also summarize the experimental status and important decays of candidate members of these multiplets, and compare the predictions for decay rates with experiment.

We start by briefly reviewing the established $1 \mathrm{~S}$ and $1 \mathrm{P}$ states that confirm that ${ }^{3} \mathrm{P}_{0}$ pair creation dominates most hadronic decays. SHO wavefunctions are employed for 
convenience; these lead to analytic results for decay amplitudes and are known to give reasonable empirical approximations. This is sufficient for our main purpose, which is to emphasize selection rules and to isolate major modes to aid in the identification of states. In addition to the $1 \mathrm{~S}$ and $1 \mathrm{P}$ states we also find reasonable agreement between the model and decays of $1 \mathrm{D}, 2 \mathrm{P}$ and $1 \mathrm{~F}$ states where data exist; this confirms the extended utility of the model and adds confidence to its applications to unknown states.

Examples of new results include the following.

- The radial $2^{3} \mathrm{P}_{1} a_{1 R} \rightarrow \rho \pi$ is strongly suppressed in $\mathrm{S}$-wave, and dominant in Dwave. This contrasts with the expectation for a hybrid $a_{1}$. The model's prediction of a dominant D-wave has been dramatically confirmed for the $a_{1}(1700)[7,8]$ and thereby establishes $1.7 \mathrm{GeV}$ as the approximate mass of the $n \bar{n}$ members of the $2 \mathrm{P}$ nonets. This includes the $0^{++}$nonet whose $\mathrm{I}=0$ members share the quantum numbers of the scalar glueball.

- In the scalar glueball sector, we find that the decays of the $f_{0}(1500)$ and the $f_{J}(1710)$ are inconsistent with radially excited quarkonia.

- We identify the $2 \mathrm{~S}^{-+}$nonet. The $\eta$ members are predicted to have narrow widths relative to the $\pi$ counterpart. This is consistent with the broad $\pi(1300)$ and the narrower candidates $\eta(1295)$ and $\eta(1440)$.

- The vector states $\rho(1465)$ and $\omega(1419)$ are interesting in that the decay branching fractions appear to show anomalous features requiring a hybrid component. We identify the experimental signatures needed to settle this question.

- The $\pi(1800)$ has been cited as a likely hybrid candidate $[2,9,10]$ on the strength of its decay fractions. The $3 \mathrm{~S}^{-+} q \bar{q} \pi$ is also anticipated in this region. We find that the decays of the hybrid and $3 \mathrm{~S}^{-+}$have characteristic differences which enable them to be distinguished. We identify modes that may enable the separation of these two configurations.

Our other results for the many $n \bar{n}$ states predicted up to $2.1 \mathrm{GeV}$ should be useful in 
the identification of these higher quarkonia, and in confirming that non-exotic gluonic or molecular states are indeed inconsistent with quarkonium assignments.

The order of discussion is $1 \mathrm{~S}$ and $1 \mathrm{P}$ (section 2); $2 \mathrm{~S}$ and ${ }^{3} D_{1}$ (section 3 ); $3 \mathrm{~S}$ (section 4); 2P (section 5); 1D (section 6); 1F (section 7). A summary and an outline for experimental strategy is in section 8 .

\section{1S AND 1P TESTBED}

First we will use the well known decays of light 1S and 1P $n \bar{n}$ states to motivate and constrain the ${ }^{3} \mathrm{P}_{0}$ decay model. Ackleh, Barnes and Swanson [11] have carried out a systematic study of $q \bar{q}$ decays in the ${ }^{3} \mathrm{P}_{0}$ and related pair creation decay models: in that work a ${ }^{3} \mathrm{P}_{0}$-type amplitude was established as dominant in most light $n \bar{n}$ decays. (For other discussions of $q \bar{q}$ decays in the ${ }^{3} \mathrm{P}_{0}$ model see Ref. [13]). Fig.1, from Ref. [11], shows ${ }^{3} \mathrm{P}_{0}$ model predictions for the decay widths. Large widths are indeed predicted to be large and smaller widths are found to be correspondingly small. If we choose the pair creation strength $\gamma=0.5$ (Eq. A3) to set an approximately correct overall width scale, then $\Gamma\left(h_{1} \rightarrow \rho \pi\right)$ and $\Gamma\left(a_{1} \rightarrow \rho \pi\right)$ are both $\approx 0.4-0.5 \mathrm{GeV} ; \Gamma\left(f_{2} \rightarrow \pi \pi\right), \Gamma(\rho \rightarrow \pi \pi)$ and $\Gamma\left(b_{1} \rightarrow \omega \pi\right)$ are all $\approx 0.1-0.2 \mathrm{GeV}$, and $\Gamma\left(a_{2} \rightarrow \rho \pi\right)$ is smallest, $\approx 0.05 \mathrm{GeV}$; all are reasonably close to the observed widths.

The optimum parameter values found in a fit to the partial widths of Fig.1 [11] are $\beta=0.40 \mathrm{GeV}$ (which is actually the length scale most commonly used in light $q \bar{q}$ decays) and $\gamma=0.51$; with these values the rms relative error for these six decays is $\Delta \Gamma / \Gamma_{\text {expt }}=$ $29 \%$. In this work we have actually found that the pair production amplitude $\gamma=0.5$ is somewhat large for higher-L $q \bar{q}$ states, so in our discussions of higher quarkonia we will instead use $\gamma=0.4$. In constrained- $\gamma$ fits we find that using $\gamma=0.4$ only moderately decreases the accuracy of the fit to the light $1 \mathrm{~S}$ and $1 \mathrm{P}$ decays, to $\Delta \Gamma / \Gamma_{\text {expt }}=43 \%$, with an optimum $\beta=0.36 \mathrm{GeV}$.

A more sensitive test of the ${ }^{3} \mathrm{P}_{0}$ model involves amplitude ratios in the decays $b_{1} \rightarrow \omega \pi$ 


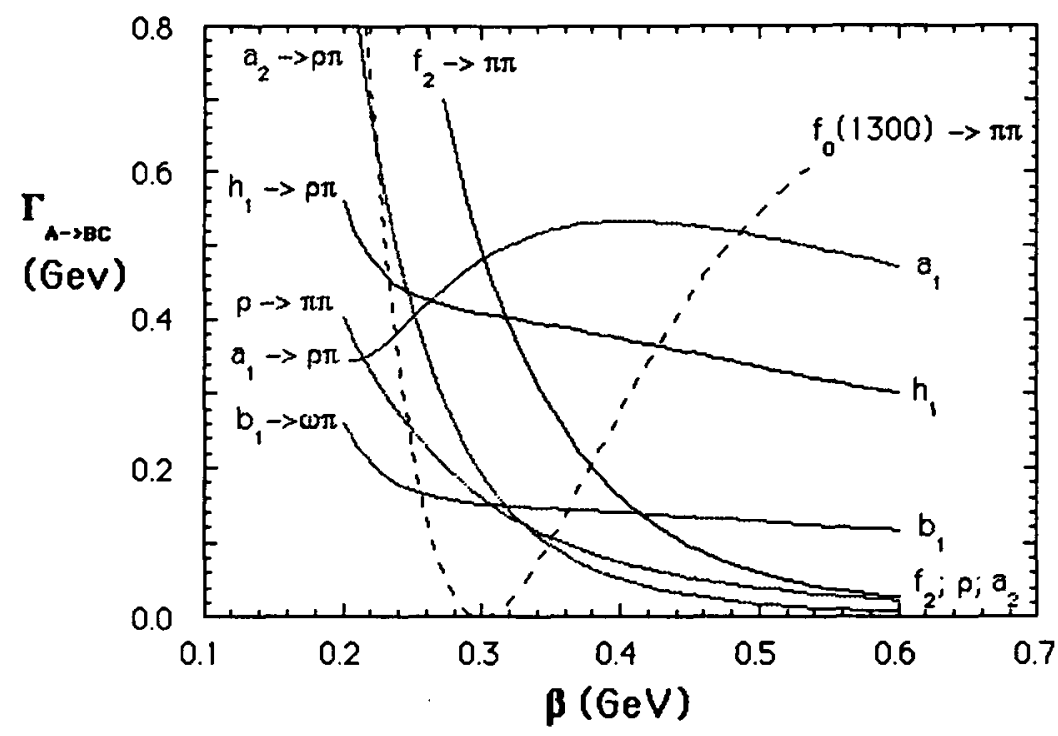

Figure 1. Partial widths of light $1 S$ and $1 \mathrm{P} q \bar{q}$ mesons in the ${ }^{3} \mathrm{P}_{0}$ model. The model parameters shown are $\beta=0.2-0.6 \mathrm{GeV}$ (with $\beta \approx 0.4 \mathrm{GeV}$ preferred) and $\gamma=0.5$.

and $a_{1} \rightarrow \rho \pi$. In these decays both S- and D-wave final states are allowed, and the ratio of these decay amplitudes is known to be $\mathrm{D} / \mathrm{S}=+0.260(35)$ for the $b_{1}$ and $-0.09(2)$ for the $a_{1}$ [14]. This ratio is quite sensitive to the quantum numbers of the produced pair; with ${ }^{3} \mathrm{P}_{0}$ quantum numbers and the usual $\beta$ we find reasonable agreement in sign and magnitude, whereas a OGE pair production mechanism gives the wrong sign for D/S [11]. This ratio test for $b_{1} \rightarrow \omega \pi$ was historically very important in establishing the ${ }^{3} \mathrm{P}_{0}$ decay model [12].

These successes of the ${ }^{3} \mathrm{P}_{0}$ model motivate its use in predicting decays of the less familiar radial and orbital excitations of light quarkonia.

\section{2S STATES}

We first consider the decays of the low-lying radially-excited pseudoscalar and vector states. Our general approach will be to review recent data on the state in question and 
compare these data to predictions for candidate $q \bar{q}$ and (where appropriate) hybrid states. In each case we will attempt to identify decay modes that distinguish between competing assignments most clearly.

$$
\text { A. } 0^{-+} 2^{1} S_{0}: \pi \text { and } \eta
$$

- $\pi(1300)$

The $\pi(1300)$ was first reported by Bellini et al. [15] in 1982 but remains rather poorly known. It is seen in $\pi \rho, \pi(\pi \pi)_{S}$ and $\pi f_{0}(1300)$, with a width of $200-600 \mathrm{MeV}$; there is however no accurate measurement of the branching fractions [16]. Recently higher statistics have been obtained for the $\pi(1300)$ by VES $[7,10]$ and by E852 at BNL [8]. The VES data shows a clear $\pi(1300)$ peak in $3 \pi$, with a width of $\Gamma \approx 400-500 \mathrm{MeV}$ in both $\pi(\pi \pi)_{S}$ and $\rho \pi$; the latter is particularly strong and dominates this channel below $2 \mathrm{GeV}$.

It should be noted, however, that the size of the Deck background in $\pi(\pi \pi)_{S}$ is uncertain, and it is not clear whether the $\pi(1300)$ reported in $\pi(\pi \pi)_{S}$ is actually due to the resonance. Fig.1c of Ref. [7] suggests that the Deck mechanism could cause all of the $\pi(1300) \rightarrow \pi(\pi \pi)_{S}$ enhancement in Fig.4a of that reference. We will assume that this is essentially correct, and that the $\pi(1300)$ resonance decays dominantly to $\rho \pi$.

In the ${ }^{3} \mathrm{P}_{0}$ decay model we expect $\rho \pi$ to be the dominant mode of a $2 S q \bar{q} \pi(1300)$, since this is the only open two-body channel. (We assume that the $f_{0}(980)$ and $a_{0}(980)$ are dominantly $\mathrm{K} \bar{K}$, so the mode $\pi(1300) \rightarrow f_{0}(980) \pi$ is a more complicated three body or virtual two-body decay.) With our parameter set $\gamma=0.4$ and $\beta=0.4 \mathrm{GeV}$ we predict a partial width of

$$
\Gamma(\pi(1300) \rightarrow \pi \rho)=209 \mathrm{MeV}
$$

This rate is given in Table B2 of Appendix B. (App.B is a tabulation of all our numerical results for partial widths in the ${ }^{3} \mathrm{P}_{0}$ model.) In Fig.2 we show the dependence of this prediction on the wavefunction length scale $\beta$. Evidently the prediction of a lárge width, 


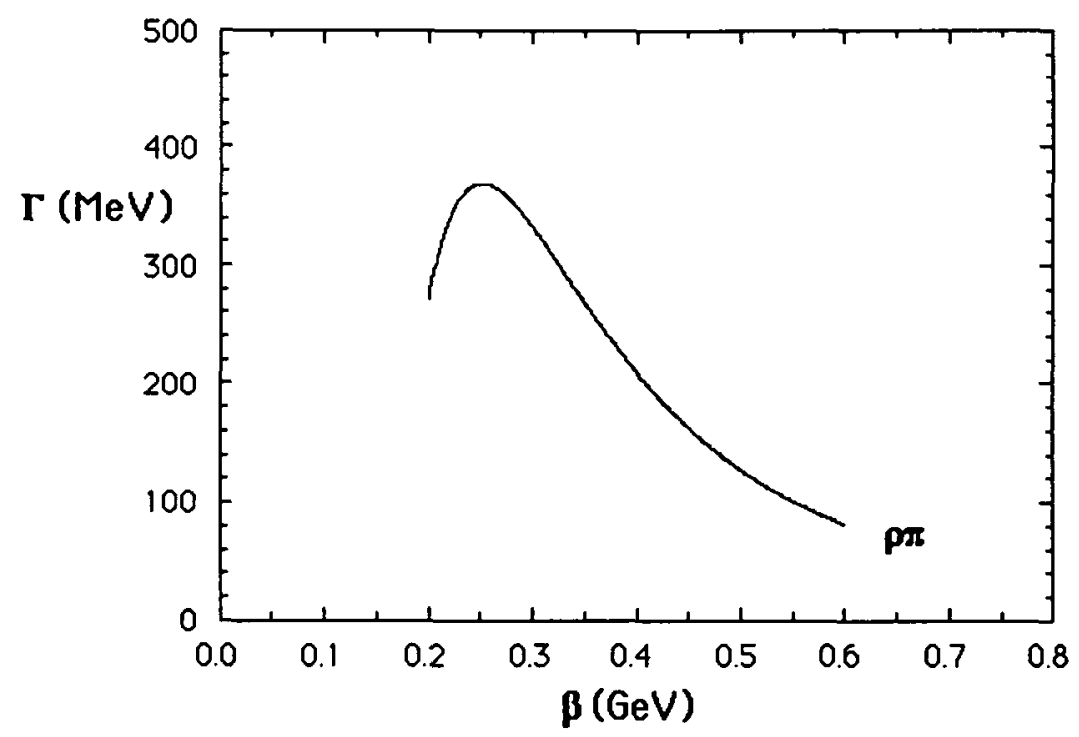

Figure 2. The $\rho \pi$ partial width of a $2 \mathrm{~S} \pi(1300)$, with ${ }^{3} \mathrm{P}_{0}$ model parameters $\beta=0.2-0.6$ $\mathrm{GeV}$ and $\gamma=0.4$.

comparable to observation, follows from any plausible choice for $\beta$. Thus the observed $\pi(1300)$ is consistent with expectations for a $2^{1} \mathrm{~S}_{0} q \bar{q}$ state.

Although the mode $f_{0}^{q \bar{q}}(1300) \pi$ is nominally closed by phase space, the $f_{0}(1300)$ is a very broad state, so one might anticipate a significant $(\pi \pi)_{S} \pi$ mode through the lowmass tail of the $f_{0}(1300)$. This possibility may be tested by varying $M\left(f_{0}^{q \bar{q}}\right)$; the resulting $\Gamma\left(\pi(1300) \rightarrow f_{0}^{q \bar{q}} \pi\right)$ does not exceed $10 \mathrm{MeV}$ over the range $M\left(f_{0}^{q \bar{q}}\right)=400-1000 \mathrm{MeV}$. Thus, the population of a $\pi(\pi \pi)_{S}$ mode by $\pi(1300)$ decays through an intermediate $f_{0}^{q \bar{q}} \pi$ state is predicted to be a small effect. If there actually is a large $\pi(1300) \rightarrow \pi(\pi \pi)_{S}$ mode, rather than a nonresonant Deck effect, this would be in disagreement with the ${ }^{3} \mathrm{P}_{0}$ model. Thus it would be very interesting to establish the branching fraction for $\pi(1300) \rightarrow \pi(\pi \pi)_{S}$ accurately in future work.

- $\eta(1295)$

This state has a width of $\Gamma=53(6) \mathrm{MeV}[16]$, much narrower than its $\mathrm{I}=12^{1} \mathrm{~S}_{0}$ 
partner $\pi(1300)$. It has been reported in $a_{0}(980) \pi$ and $\eta \pi \pi$. This small width is natural if the $\pi(1300)$ does indeed decay dominantly to $\rho \pi$, since G-parity forbids the analogous processes $\eta_{n \bar{n}} \rightarrow \rho \pi$ and $\eta_{n \bar{n}} \rightarrow \omega \eta$; to the extent that the $a_{0}(980)$ and $f_{0}(980)$ are dominantly $K \bar{K}$ there are no quasi-two-body $q \bar{q}$ modes open to the $\eta(1295)$. Consequently the decays must proceed through the weaker direct three-body and virtual two-body channels such as $a_{0}^{q \bar{q}} \pi$ and $f_{0}^{q \bar{q}} \eta$.

It is interesting to note the rôle that the $2 \mathrm{~S}$ initial wavefunction has played in our discussion. Suppose for illustration that we had instead used 1S wavefunctions for the $\pi(1300)$ and $\eta(1295)$; we would then have predicted partial widths of several hundred $\mathrm{MeV}$ into the low-energy tails of the modes $f_{0}^{q \bar{q}} \pi$ and $a_{0}^{q \bar{q}} \pi$, with consequent broad widths for the $\pi(1300)$ and the $\eta(1295)$, in contradiction with experiment.

- $\eta(1440)$

These successes raise provocative questions regarding the $\eta(1440)$ state(s). This is a purportedly complicated region which may contain more than one resonance [16]. The PDG width of the $\eta(1440)$ is only $\Gamma=60(30) \mathrm{MeV}$, with signals reported in $K^{*} K$, $a_{0}(980) \pi, \eta(\pi \pi)_{S}$ and $\rho \gamma$.

Except for $\rho \gamma$ these modes are not inconsistent with a dominantly $s \bar{s}$ state. The only two-body strong channel open for a $2^{1} \mathrm{~S}_{0} s \bar{s} \eta(1440)$ is $K^{*} K$, but this could rescatter from KK $\pi$ into the other reported modes $a_{0}(980) \pi$ and $\eta \pi \pi$. The ${ }^{3} \mathrm{P}_{0}$ model prediction for the partial width $\eta(1440) \rightarrow K^{*} K$ versus the wavefunction length scale $\beta$ is shown in Fig.3. Evidently the predicted $K^{*} K$ partial width is comparable to the observed width, so a $2^{1} \mathrm{~S}_{0} s \bar{s}$ assignment appears possible for this state.

Of course the $\rho \gamma$ mode is not expected from $s \bar{s}$, and if confirmed may imply large $n \bar{n} \leftrightarrow s \bar{s}$ mixing in this sector as is observed in the $1 \mathrm{~S} I=0$ pseudoscalars. This can be parameterized as

$$
\begin{aligned}
|\eta(1295)\rangle & =+\cos (\theta)|n \bar{n}\rangle+\sin (\theta)|s \bar{s}\rangle \\
|\eta(1440)\rangle & =-\sin (\theta)|n \bar{n}\rangle+\cos (\theta)|s \bar{s}\rangle .
\end{aligned}
$$




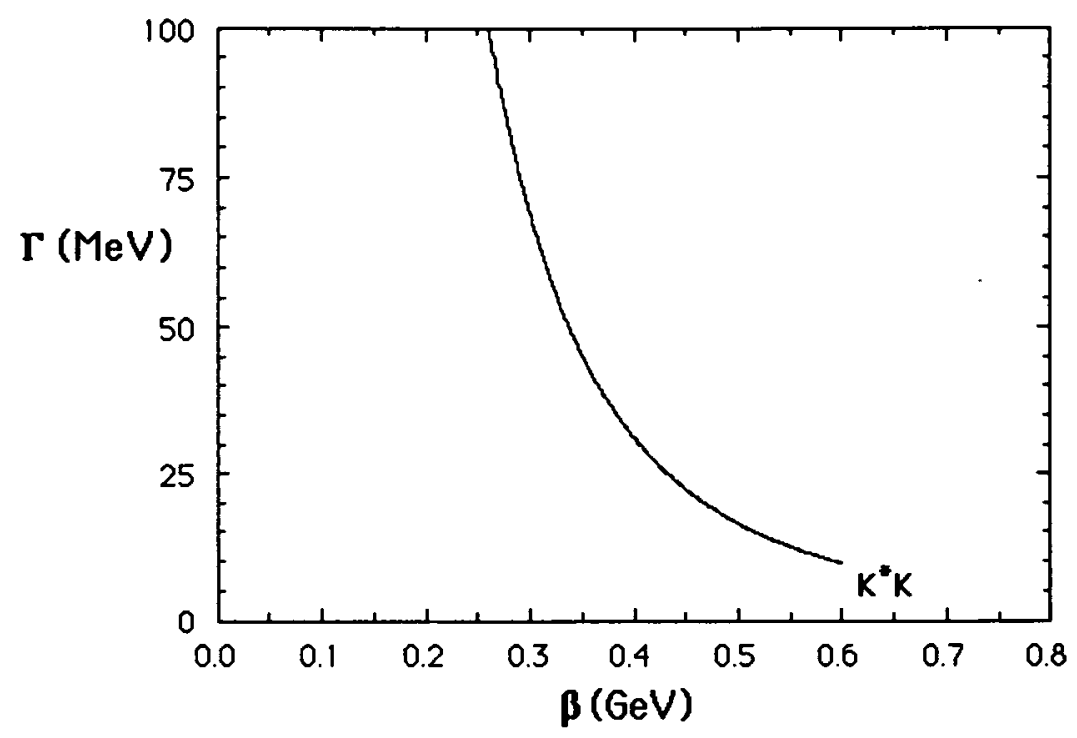

Figure 3. The $\mathrm{K}^{*} \bar{K}+$ h.c. partial width of a $2{ }^{1} \mathrm{~S}_{0} s \bar{s} \eta(1440)$ in the ${ }^{3} \mathrm{P}_{0}$ model. Other two-body modes are excluded by phase space.

A remeasurement of $\eta(1440) \rightarrow \rho \gamma$, which should be possible at BEPC and TCF in $\psi \rightarrow \gamma \gamma \rho$, would be very useful in clarifying the nature of this state. Ideally we would like to know the invariant mass distributions of $\rho \gamma, \omega \gamma$ and $\phi \gamma$ final states, since these are flavor-tagging modes that allow investigation of possible flavor mixing in the parent resonances. Similarly, an accurate measurement of the branching fractions in the flavortagging $\psi \rightarrow V \eta(1440)$ and $V \eta(1295)$ hadronic decays, with $V=\omega, \phi$, would be useful for the determination of the $n \bar{n}-s \bar{s}$ mixing angle.

In summary, from the total widths alone it is possible to describe the $\eta(1295)$ and $\eta(1440)$ as unmixed $n \bar{n}$ and $s \bar{s} 2^{1} \mathrm{~S}_{0}$ radial excitations. The report of a large $\eta(1440) \rightarrow$ $\rho \gamma$ radiative mode however suggests flavor mixing between these states, and should be remeasured with greater sensitivity together with other $V \gamma$ modes. This mixing could also account for the large $\eta(1440)$ signal seen in $\eta(\pi \pi)$ by GAMS [17]. 


\section{B. $1^{--}: 2^{3} S_{1}$ and ${ }^{3} D_{1} \rho$ and $\omega$}

$$
\bullet(1465), \rho(1700)
$$

If one accepts that the $\pi(1300)$ and $\eta(1295)$ belong to a $2^{1} \mathrm{~S}_{0} q \bar{q}$ nonet, it is then natural to assign the $\rho(1465)$ and the $\omega(1419)[16,18]$ to $2^{3} \mathrm{~S}_{1}$ states. Indeed, one expects the contact hyperfine interaction to raise the mass of the vector nonet with respect to the pseudoscalar nonet by approximately this amount [19]. It is unlikely that the vectors near 1.4-1.5 GeV are dominantly $\mathrm{D}$-waves, since the ${ }^{3} \mathrm{D}_{1} n \bar{n}$ states should lie close to the other $1 \mathrm{D}$ candidates such as the $\pi_{2}(1670), \rho_{3}(1691)$ and $\omega_{3}(1667)$. In the Godfrey-Isgur potential model a mass of $1660 \mathrm{MeV}$ was predicted for the ${ }^{3} \mathrm{D}_{1}$ state, whereas they expect the $2^{3} \mathrm{~S}_{1}$ radial excitation at $1450 \mathrm{MeV}$ [19]. The $\rho(1465)$ also lies well below flux-tube model expectations of $\mathrm{M}_{H}\left(1^{--}\right) \approx 1.8-1.9 \mathrm{GeV}[3,4]$ for vector hybrids, so although the possibility of light vector hybrids has been discussed $[2,20]$, these do not appear likely unless the flux tube model for hybrids is misleading.

The experimental branching fractions of these $1^{--}$states are somewhat obscure, because there are at least two broad, overlapping resonances in each flavor sector in this mass region. The status of these vector states as seen in $e^{+} e^{-}$annihilation was reviewed recently by Clegg and Donnachie [18]. In the $\rho$ sector they find that at least two states are present. The lighter state is assigned a mass of $\mathrm{M}=1.463(25) \mathrm{GeV}$ and a width of $\Gamma=0.311(62) \mathrm{GeV}$; it couples strongly to $4 \pi$ states (including $a_{1} \pi$ but not $h_{1} \pi$ ) and $\omega \pi$, and less strongly to $\pi \pi$. The higher state has $\mathrm{M}=1.73(3) \mathrm{GeV}, \Gamma=0.40(10) \mathrm{GeV}$, couples most strongly to $4 \pi$ ( $a_{1} \pi$ and $h_{1} \pi$ are not separated) and perhaps $6 \pi ; \pi \pi$ is also important, but the $\omega \pi$ width is found to be small.

These states have also been reported recently by Crystal Barrel [21] in $\pi^{-} \pi^{o}$ states in $\bar{p} d \rightarrow \pi^{-} \pi^{o} \pi^{o} p ;$ both vectors appear in $\pi^{-} \pi^{o}$, with masses and widths of $M=1.411(10)(10)$ $\mathrm{GeV}, \Gamma=0.343(18)(8) \mathrm{GeV}$, and $\mathrm{M}=1.780_{-25}^{+34}(14) \mathrm{GeV}, \Gamma=0.275(42)(17) \mathrm{GeV}$, quite similar to the $e^{+} e^{-}$results.

The ${ }^{3} \mathrm{P}_{0}$ model predictions for pure $2^{3} \mathrm{~S}_{1}$ and ${ }^{3} \mathrm{D}_{1} \rho$ states at $1.465 \mathrm{GeV}$ and $1.700 \mathrm{GeV}$ 
TABLE I. Partial widths of $2 \mathrm{~S}, 1 \mathrm{D}$ and hybrid $\rho$ states.

\begin{tabular}{lccccccccc}
\hline \hline & $\pi \pi$ & $\omega \pi$ & $\rho \eta$ & $\rho \rho$ & $\mathrm{KK}$ & $\mathrm{K}^{*} \mathrm{~K}$ & $h_{1} \pi$ & $a_{1} \pi$ & total \\
\hline$\rho_{2 S}(1465)$ & 74. & 122. & 25. & - & 35. & 19. & 1. & 3. & 279. \\
$\rho_{1 D}(1700)$ & 48. & 35. & 16. & 14. & 36. & 26. & 124. & 134. & 435. \\
$\rho_{H}(1500)$ & 0 & 5 & 1 & 0 & 0 & 0 & 0 & 140 & $\approx 150$ \\
\hline \hline
\end{tabular}

are given in Table I (see also Tables B1, B8), together with flux tube model predictions for a hypothetical $1.5 \mathrm{GeV}$ vector hybrid. Very characteristic differences between the states are evident in their couplings to $4 \pi$ final states; $2 \mathrm{~S}$ couples very weakly to these, 1D couples strongly to both $a_{1} \pi$ and $h_{1} \pi$, and the hybrid couples strongly to $a_{1} \pi$ but not to $h_{1} \pi$. Both quarkonium states have moderately large couplings to $\pi \pi$ and $\omega \pi$, whereas the hybrid couples strongly only to $a_{1} \pi$.

Note that the $|q \bar{q}\rangle$ components are spin triplet whereas the hybrid is spin singlet. This difference in spin underlies the characteristic pattern of branching fractions in Tables I and II.

Although there are many similarities between theory and experiment, there are problems in detail. The important couplings of the lighter state to $\pi \pi$ and $\omega \pi$ found by Clegg and Donnachie are consistent with a $2 \mathrm{~S}$ quarkonium, but we do not expect a significant coupling of a $2^{3} \mathrm{~S}_{1} \rho$ to $4 \pi$ final states. The dominant coupling of the heavier state to $4 \pi$ is as predicted for the $\mathrm{D}$-wave quarkonium, but the reported absence of $\omega \pi$ is not expected. The presence of two states $\left(2^{3} \mathrm{~S}_{1}\right.$ and $\left.{ }^{3} \mathrm{D}_{1}\right)$ in $\pi \pi$ with comparable strengths, reported by Crystal Barrel [21], is expected.

Of course it is difficult to distinguish the contributions from two broad states with similar masses, and the $4 \pi$ final states themselves have not yet been completely characterized. (The $a_{1} \pi$ and $h_{1} \pi$ modes of the $\rho(1700)$ in $e^{+} e^{-}$for example have not been separated.) It appears likely that the states and their branching fractions are still inadequately resolved experimentally in this mass region, so it is not yet appropriate to attempt a detailed fit, 
TABLE II. Partial widths of $2 \mathrm{~S}, 1 \mathrm{D}$ and hybrid $\omega$ states.

\begin{tabular}{lcccccc}
\hline \hline & $\rho \pi$ & $\omega \eta$ & $\mathrm{KK}$ & $\mathrm{K}^{*} \mathrm{~K}$ & $b_{1} \pi$ & total \\
\hline$\omega_{2 S}(1419)$ & 328. & 12. & 31. & 5. & 1. & 378. \\
$\omega_{1 D}(1649)$ & 101. & 13. & 35. & 21. & 371. & 542. \\
$\omega_{H}(1500)$ & 20 & 1 & 0 & 0 & 0 & $\approx 20$ \\
\hline \hline
\end{tabular}

using for example linear combinations of the $2 \mathrm{~S}$ and $1 \mathrm{D}$ basis states.

It is clear from our ${ }^{3} \mathrm{P}_{0}$ results that in future it will be important to separate the $a_{1} \pi$ and $h_{1} \pi$ contributions (which tag $1 \mathrm{D}$ and $\mathrm{H}[2,20]$ states), and that the $\pi \pi$ and $\omega \pi$ distributions should also be studied carefully, since these are expected to arise mainly from quarkonia rather than hybrids.

- $\omega(1419)$ and $\omega(1649)$

We anticipate similar problems with at least two broad overlapping resonances in the $\mathrm{I}=0$ sector. Clegg and Donnachie [18] discuss both one- and two-resonance fits to the $\omega$ sector in the reactions $e^{+} e^{-} \rightarrow \rho \pi$ and $\omega \pi \pi$. In their two-resonance fit they find a lower state with a mass and width of $\mathrm{M}=1.44(7) \mathrm{GeV}, \Gamma=0.24(7) \mathrm{GeV}$, and a higher, quite narrow state with $\mathrm{M}=1.606(9) \mathrm{GeV}, \Gamma=0.113(20) \mathrm{GeV}$. The PDG quote masses and widths of $\mathrm{M}=1.419(31) \mathrm{GeV}, \Gamma=0.174(59) \mathrm{GeV}, \mathrm{M}=1.649(24) \mathrm{GeV}, \Gamma=0.220(35)$ $\mathrm{GeV}$; the parameters for the lighter state are consistent but the width of the higher-mass $\omega$ state is broader than Clegg and Donnachie estimate.

Clegg and Donnachie find that both $\omega$ states couple strongly to $\rho \pi$. Only the second is found to couple to $\omega \pi \pi$, and that coupling is rather weak. A fit with a single resonance finds instead that the $\omega \pi \pi$ branching fraction exceeds $\rho \pi$, so these should be regarded as tentative conclusions.

For comparison we again show the numerical predictions of the ${ }^{3} \mathrm{P}_{0}$ model for pure 2S, $1 \mathrm{D}$ and $\mathrm{H}$ states. The masses assumed are $1996 \mathrm{PDG}$ values (see Tables $\mathrm{B} 1$ and B9). The large $\rho \pi$ couplings reported for the vector states are evidently consistent with 
expectations for both $2 \mathrm{~S}$ and $1 \mathrm{D}$ quarkonia. Again the $\mathrm{S}+\mathrm{S}$ modes are predicted to be small for a hybrid, so they can be used to tag quarkonia or the $q \bar{q}$ components of mixed states. Since none of the favored $\mathrm{S}+\mathrm{P}$ modes is open to an $\mathrm{I}=0$ hybrid at $1.5 \mathrm{GeV}$, such a state would be quite narrow, as shown in Table II. (The decay $\omega_{H} \rightarrow b_{1} \pi$ is excluded by the "singlet selection rule" $[2,11]$, which states that $\left(S_{q \bar{q}}=0\right) \nrightarrow\left(S_{q \bar{q}}=0\right)+\left(S_{q \bar{q}}=0\right)$ in the ${ }^{3} \mathrm{P}_{0}$ model; the $\omega_{H}$ hybrid has $S_{q \bar{q}}=0$ in the flux tube model. Interestingly, the singlet selection rule holds for both ${ }^{3} \mathrm{P}_{0}$ and $\mathrm{OGE}$ quarkonium decay amplitudes [11].)

A hybrid in this mass region should be visible as a narrow bump in the $\rho \pi$ invariant mass distribution. (This channel is not favored for a hybrid, but it is allowed at a reduced rate due to different $\rho$ and $\pi$ spatial wavefunctions.) Thus it may be useful to search $\rho \pi$ final states for narrow resonances with improved statistics, although the signal would of course be broadened by the $\rho$ width.

The very large $b_{1} \pi$ mode predicted for the $1 \mathrm{D}$ quarkonium is very interesting, because neither $2 \mathrm{~S}$ nor hybrid vector states are expected to couple significantly to $b_{1} \pi$. This twobody mode will appear as $\omega \pi \pi$; Clegg and Donnachie do report an $\omega \pi \pi$ mode for their higher $\omega$ state, but the coupling is not as strong as we predict. The total width of their higher-mass state is also much smaller than expected. Since the 1D state is predicted to have a very large width, $\approx 500 \mathrm{MeV}$ (Table B9), this discrepancy may be due to a distortion of the shape by threshold effects, with resulting inaccuracies in the reported couplings. Assuming that the ${ }^{3} \mathrm{P}_{0}$ model predictions are approximately correct, a study of the $1^{--} \omega \pi \pi$ mass distribution should reveal the ${ }^{3} \mathrm{D}_{1} \omega$ basis state in isolation. (It may be distributed over several resonances.) If the quasi-two-body approximation is correct, the mass distribution of $\omega \pi$ pairs in the resonance contribution to $\omega \pi \pi$ should be consistent with a $b_{1}(1231)$. 


\section{Mixing in the $1^{--}$sector.}

Although we have considered the decay modes of pure $2 \mathrm{~S}, 1 \mathrm{D}$ and $\mathrm{H}$ vector states, the physical resonances are certainly linear combinations of these and other basis states. Since the known resonances have similar masses, we should consider the possibility that there is significant mixing and introduce the linear combination

$$
\left.|V\rangle=\cos (\theta)\left(\cos (\phi)\left|2^{3} S_{1}\right\rangle+\left.\sin (\phi)\right|^{3} D_{1}\right\rangle\right)+\sin (\theta)|H\rangle
$$

The mixing angles for each resonance can be determined from the branching fractions to certain states. The $\mathrm{S}+\mathrm{S}$ modes identify the $q \bar{q}$ components of the state (see Tables I and II). In the I=1 states the $4 \pi$ modes $a_{1} \pi$ and $h_{1} \pi$ are similarly characteristic; the $h_{1} \pi$ mode is produced only by the $1 \mathrm{D}$ basis state, and $a_{1} \pi$ comes from both $1 \mathrm{D}$ and hybrid states. Similarly in $\mathrm{I}=0$ the mode $b_{1} \pi$ tags the $1 \mathrm{D}$ quarkonium basis state and $2 \mathrm{~S}$ and 1D states both lead to strong $\rho \pi$ couplings. Determination of the mixing angles in the physical states will be possible given accurate measurements of the branching fractions to these characteristic modes.

We have not carried out a fit to determine the mixing angles because the experimental results do not yet appear definitive. However we note that the partial widths reported by Clegg and Donnachie for the $\rho(1465)$, which include a large $\Gamma_{a_{1} \pi}$ and a small $\Gamma_{h_{1} \pi}$, are inconsistent with $2 \mathrm{~S}$ or $1 \mathrm{D}$ alone. These widths imply a large $\mathrm{H}$ component in this state with the possibility of considerable $\mathrm{H}-2 \mathrm{~S}$ mixing.

Future experimental work could concentrate on an accurate determination of the $\pi \pi$, $\omega \pi, h_{1} \pi$ and $a_{1} \pi$ branching fractions of the $\rho$ states. The $h_{1} \pi$ and $a_{1} \pi$ modes are especially sensitive to the nature of the initial state. Similarly the $\rho \pi$ and $b_{1} \pi$ branching fractions of the $\omega$ states are the most interesting experimentally. 
TABLE III. Partial widths of $3 S$ and hybrid $\pi(1800)$ states.

\begin{tabular}{lccccccc}
\hline \hline & $\rho \pi$ & $\rho \omega$ & $\rho(1465) \pi$ & $f_{0}(1300) \pi$ & $f_{2} \pi$ & $\mathrm{K}^{*} \mathrm{~K}$ & total \\
\hline$\pi_{3 S}(1800)$ & 30. & 74. & 56. & 6. & 29. & 36. & 231. \\
$\pi_{H}(1800)$ & 30 & 0 & 30 & 170 & 6 & 5 & $\approx 240$ \\
\hline \hline
\end{tabular}

\section{3S STATES}
A. $0^{-+}: 3^{1} \mathbf{S}_{0} \pi(1800)$

The same experiments $[7,10,15,22]$ that see the $\pi(1300)$ in $\rho \pi$ and a possible broad enhancement in $\pi(\pi \pi)_{S}$ also report a prominent $\pi(1800)$ in $f_{0}(980) \pi, f_{0}(1300) \pi, f_{0}(1500) \pi$ and $K(K \pi)_{s}$. None of these experiments see the $\pi(1800)$ in $\rho \pi$. This is striking, as also is the fact that the total width of $\approx 150-200 \mathrm{MeV}$ is considerably smaller than that of the $\pi(1300)$. Furthermore, the presence of clear signals in both $f_{0}(1300) \pi$ and $f_{0}(980) \pi$ is remarkable and was commented upon with some surprise [10].

The decays into $\pi \rho$ and $\mathrm{KK}^{*}$ are both suppressed; VES quote the limits [10]

$$
\frac{\pi(1800) \rightarrow \pi^{-} \rho^{0}}{\pi(1800) \rightarrow \pi^{-} f_{0}(980)_{\mid \rightarrow \pi^{+} \pi^{-}}}<0.14 \quad(90 \% \text { c.l. })
$$

and

$$
\frac{\pi(1800) \rightarrow K^{-} K^{*}}{\pi(1800) \rightarrow K^{-} K^{+} \pi(S-\text { wave })}<0.1 \quad(95 \% \text { c.l. })
$$

A prominent $\mathrm{KK}_{0}^{*}$ signal is present (observed as $K(K \pi)_{S}$ ), so the virtual transition $\pi(1800) \rightarrow K K_{0}^{*} \rightarrow K K \pi \rightarrow f_{0}(980) \pi$ is probably responsible for the coupling to $f_{0}(980) \pi$; this mode appears to be stronger than $f_{0}(1300) \pi$. The mass of this state makes it a candidate for either the radial $3^{1} \mathrm{~S}_{0}$ or the ground state hybrid $\pi_{H}$. The predicted branching fractions for $3^{1} \mathrm{~S}_{0}$ (Table B4) and $\pi_{H}$ hybrid states (from Ref. [2]) near this mass are shown in Table III.

The decay amplitude for $3{ }^{1} \mathrm{~S}_{0} \rightarrow{ }^{3} \mathrm{~S}_{1}+{ }^{1} \mathrm{~S}_{0}$ is actually close to a node with these masses, so the weak coupling to $\rho \pi$ is expected for both a $3 S$ quarkonium and a hybrid. The most 
important differences are in the $\rho \omega$ and $f_{0}(1300) \pi$ modes: $\rho \omega$ is predicted to be the largest mode of a $3 S \pi(1800)$ state, whereas for a hybrid $\pi_{H}(1800) \rightarrow \rho \omega$ should be very weak (this is the usual selection rule against $S+S$ final states). Conversely, $f_{0}(1300) \pi$ is predicted to be weak for 3S quarkonium but is expected to be the dominant decay mode of a $\pi_{H}(1800)$ hybrid. The observation of a large $f_{0}(1300) \pi$ mode argues in favor of a hybrid assignment for this state. One should note however that the ${ }^{3} \mathrm{P}_{0}$ model also predicts a small branching fraction for $\pi(1300) \rightarrow \pi(\pi \pi)_{S}$; if the observed $\pi(\pi \pi)_{S}$ signal is really due to the $\pi(1300)$ rather than the Deck effect, the decay model may simply be inaccurate for $\mathrm{N}^{1} \mathrm{~S}_{0} \rightarrow{ }^{1} \mathrm{~S}_{0}+{ }^{3} \mathrm{P}_{0}$ transitions. There may for example be large OGE decay amplitudes in these channels, as was found in the related transition ${ }^{3} \mathrm{P}_{0} \rightarrow{ }^{1} \mathrm{~S}_{0}+{ }^{1} \mathrm{~S}_{0}$ [11]; this can be checked in a straightforward calculation [23]. Thus the presence of a strong $\pi(1800) \rightarrow f_{0}(1300) \pi$ mode is indicative of a hybrid assuming that the ${ }^{3} \mathrm{P}_{0}$ model is accurate.

Although the strong $f_{0}(1300) \pi$ signal in the VES data may well have isolated the $\pi_{H}(1800)$ hybrid, VES also finds evidence for a large $\rho \omega$ signal at a similar mass [24]. We expect $\rho \omega$ to arise from the $3 S \pi(1800)$ quarkonium state rather than from a hybrid. These signals may be due to two different resonances; the $\rho \omega$ signal is evident well below $1800 \mathrm{MeV}$, and persists to higher mass than the $f_{0}(1300) \pi$ distribution. Similarly the mode $f_{2} \pi$ is observed (Fig.4d of Ref. [7]), but at a mass of $\approx 1700 \mathrm{MeV}$, well below the $\pi(1800)$ seen in $f_{0}(1300) \pi$. This may also indicate a 3S state somewhat below a hybrid $\pi(1800)$. If two $0^{-+} \pi$ resonances were to be isolated in this region, this would be strong evidence through overpopulation for both a hybrid and a $3 S q \bar{q}$ excitation.

Further investigation of the modes $\rho \pi, \rho(1465) \pi, \rho \omega, f_{0}(1300) \pi$ and $f_{2} \pi$ could be useful to clarify the resonances in the region of the $\pi(1800)$; establishing the branching fractions to these states is especially important. The most characteristic are $\rho \omega$ and $f_{0}(1300) \pi$, since the hybrid and $3 S$ quarkonium predictions differ greatly for these modes. Theoretical studies of the stability of the decay amplitudes under variation of parameters 
and wavefunctions and the assumed decay mechanism [11] would also be interesting.

Searches for the multiplet partners of this state may be useful, since they too have characteristic decay modes. A 3S $n \bar{n} \eta(1800)$ quarkonium for example (Table B4) is predicted to have large $\rho \rho$ and $\omega \omega$ modes, which should be zero for a hybrid. An $\eta(1760)$ which couples to $\rho \rho$ and $\omega \omega$ was reported by MarkIII [25] and by DM2 [26]. The conclusions regarding the presence of this pseudoscalar signal in the MarkIII $4 \pi$ data have since been disputed [27].

\section{B. $1^{--}: 3^{3} \mathbf{S}_{1}$}

If the $\pi(1800)$ is a $3 S$ quarkonium we should expect to find $3 S$ vector states near $1.9 \mathrm{GeV}$. No candidates for these states are known at present below $2.1 \mathrm{GeV}$, however there are possible $\rho$ candidates at 2150 and $2210 \mathrm{MeV}$ [16]. The predictions for decays of $3 \mathrm{~S}$ vectors are given in Table B3; it is notable that the simple $\mathrm{S}+\mathrm{S}$ modes have small couplings, with the exception of $\rho(1900) \rightarrow \rho \rho$. Unfortunately the relatively obscure $2 \mathrm{~S}+\mathrm{S}$ modes are favored, especially for the $\omega(1900)$. Some S+P modes have sufficiently strong couplings to the $3 S$ vectors to be attractive experimentally, notably $\rho(1900) \rightarrow a_{2} \pi$ and $\omega(1900) \rightarrow b_{1} \pi$. As noted previously, the $b_{1} \pi$ mode is forbidden to an $\omega$ vector hybrid by the singlet selection rule, since this hybrid decay would have $S_{q \bar{q}}=0$ for all states.

\section{2P STATES}

The 2P states are especially important because the expected mass of this multiplet $(\approx 1700 \mathrm{MeV})$ is close to the predicted mass of the lowest hybrid multiplet in the flux tube model, $\approx 1.8-1.9 \mathrm{GeV}[3,4]$. Furthermore, the position of the $1 \mathrm{P}$ and $2 \mathrm{P}$ unmixed $n \bar{n}$ levels and the 1P $s \bar{s}$ level are needed for input to quarkonium - glueball mixing studies [28] based on the lattice expectations for glueballs in this region [5]. Determining the nature of the $f_{J}(1710)$ will be important in this regard. Since the quantum numbers $1^{++}$ 
TABLE IV. Partial widths of $2 \mathrm{P}$ and hybrid $a_{1}(1700)$ states.

\begin{tabular}{cccccccccc}
\hline \hline & $\rho \pi$ & $\rho \omega$ & $\rho(1465) \pi$ & $b_{1} \pi$ & $f_{0}(1300) \pi$ & $f_{1} \pi$ & $f_{2} \pi$ & $K^{*} \mathrm{~K}$ & total \\
\hline$a_{1(2 P)}(1700)$ & 57. & 15. & 41. & 41. & 2. & 18. & 39. & 33. & 246. \\
$a_{1(H)}(1700)$ & 30 & 0 & 110 & 0 & 6 & 60 & 70 & 20 & $\approx 300$ \\
\hline \hline
\end{tabular}

and $1^{+-}$occur in both the hybrid and $2 \mathrm{P}$ multiplets, these states need to be identified to avoid confusion with hybrids. As we shall see, a recently discovered $1^{++}$state, the $a_{1}(1700)$, appears to be our first confirmed member of the $2 \mathrm{P}$ multiplet, in that it passes a very nontrivial ${ }^{3} \mathrm{P}_{0}$ model amplitude test and thereby for the first time establishes the mass scale of the $2 \mathrm{P}$ multiplets.

\section{A. $1^{++}: 2^{3} \mathbf{P}_{1} a_{1}(1700)$}

A recent experiment at BNL [29] reported a candidate $1^{-+}$exotic, produced by $\pi \rho$ and decaying to $\pi f_{1}$. They also see a $1^{++}$state in this channel at $\approx 1.7 \mathrm{GeV}$, with a width of $\approx 0.4 \mathrm{GeV}$; the relative phase of the $1^{++}$and $1^{-+}$waves was used to support the claim of a resonant $1^{-+}$. A similar $1^{++}$signal has been reported by VES in $\rho \pi[7,10]$.

The challenge is to establish whether this $1^{++} a_{1}(1700)$ is a hybrid $a_{1(H)}$ (perhaps a partner of the reported $1^{-+}$exotic) or a radial $2^{3} \mathrm{P}_{1} n \bar{n}$ state. The predicted total width of a $1^{++} a_{1}(1700)$ hybrid in the model of Close and Page [2] is $\approx 300 \mathrm{MeV}$, comparable to the observed width. However the total width predicted for a $a_{1}(1700) 2^{3} \mathrm{P}_{1} n \bar{n}$ state is similar, about $250 \mathrm{MeV}$ (see Table B5). Some differences between these assignments are evident when we compare partial widths (see Table IV). Clearly the $2 \mathrm{P}$ state couples more strongly to $\mathrm{S}+\mathrm{S}$ modes than does the hybrid, as usual, so an accurate determination of the branching fractions to $\rho \pi$ and $\rho \omega$ would be interesting. The other modes are less characteristic with the exception of $b_{1} \pi$, which should come exclusively from the quarkonium state. The absence of the decay $a_{1(H)} \rightarrow b_{1} \pi$ is a special case of the singlet selection rule cited previously as forbidding the transition $\omega_{H} \rightarrow b_{1} \pi$. We therefore 


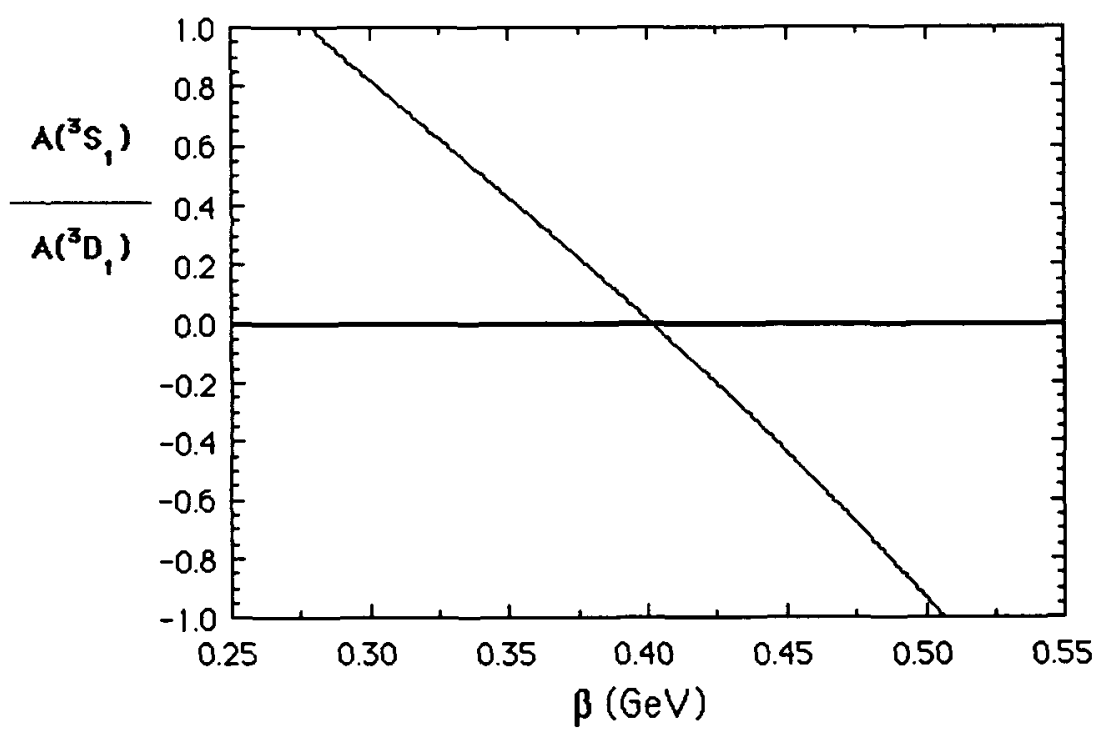

Figure 4. The S/D amplitude ratio in the transition $2^{3} \mathrm{P}_{1} a_{1}(1700) \rightarrow \rho \pi$ predicted by the ${ }^{3} \mathrm{P}_{0}$ model.

urge that experiments that observe $a_{1}(1700) \rightarrow \pi f_{1}$ also seek a signal, or a limit, for $a_{1}(1700) \rightarrow \pi b_{1}$.

A crucial test of $2 \mathrm{P}$ versus $\mathrm{H}$ assignments for the $a_{1}(1700)$ arises in the decay amplitudes to $\rho \pi$. From Appendix A, Eqs.(A53,A58,A59), the transition $2^{3} P_{1} \rightarrow{ }^{3} S_{1}+{ }^{1} S_{0}$ has both $\mathrm{S}$ and $\mathrm{D}$ amplitudes, and the $\mathrm{D} / \mathrm{S}$ ratio is (where $x \equiv\left|\vec{p}_{f}\right| / \beta$ )

$$
\left.\frac{D}{S}\right|_{2^{3} P_{1} \rightarrow{ }^{3} S_{1}+{ }^{1} S_{0}}=-\frac{2^{1 / 2} 7}{3^{2} 5} \frac{x^{2}\left(1-\frac{2}{21} x^{2}\right)}{\left(1-\frac{4}{9} x^{2}+\frac{4}{135} x^{4}\right)} .
$$

The inverse of this ratio is shown versus $\beta$ in Fig.4; note that the S-wave amplitude has a zero very close to the preferred value $\beta=0.4 \mathrm{GeV}$. This is a striking and unusual result, since in most cases we find that the lower partial waves are dominant. In contrast, for a hybrid one expects S-wave dominance, $a_{1(H)} \rightarrow(\rho \pi)_{S}:(\rho \pi)_{D} \approx 20: 1$.

Experimentally, VES sees the $a_{1}(1700)$ prominently in the $\rho \pi$ D-wave (see Fig.2c of Ref. [7]); the resonance near $1.7 \mathrm{GeV}$ dominates the entire $1-2 \mathrm{GeV}$ region. In contrast, the $\rho \pi \mathrm{S}$-wave (Fig.2a of [7]) is dominated by the $a_{1}(1230)$ and shows no clear evidence 
for the $a_{1}(1700)$. E852 similarly sees this resonance clearly in the $\rho \pi \mathrm{D}$-wave, with a mass and width of $\mathrm{M} \approx 1.66 \mathrm{GeV}$ and $\Gamma \approx 0.22 \mathrm{GeV}[8]$. This D-wave dominance of the $\rho \pi$ final state appears to be dramatic confirmation that the $a_{1}(1700)$ is a $2^{3} \mathrm{P}_{1}$ radial excitation. Furthermore the successful predictions of $a_{1} \rightarrow \rho \pi$ being in S wave and $a_{1 R} \rightarrow \rho \pi$ being in $\mathrm{D}$ wave supports the extension of the model to radial excitations.

With the $a_{1}(1700)$ established as a $2 \mathrm{P} n \bar{n}$ state, the multiplet partners are expected nearby in mass (multiplet splittings due to spin-orbit and tensor forces appear to be small even at $\mathrm{L}_{q \bar{q}}=1$ ) and searches for these states should be carried out. In the next sections we will discuss the decay modes predicted for these other $2 \mathrm{P}$ states.

B. $0^{++}, 2^{++} 2^{3} \mathbf{P}_{0}, 2^{3} \mathbf{P}_{2}: a_{0}(1700), a_{2}(1700)$

With the $a_{1}(1700)$ as the $2^{3} \mathrm{P}_{1}$ " $a_{1 R}$ " radial state, one may ask why the $a_{0 R}$ and $a_{2 R}$ partners are not seen in the same experiments. A simple explanation follows from the partial widths shown in Table B5. Since the production mechanism of the $a_{1}(1700)$ in $\pi p \rightarrow \pi f_{1} p$ apparently involves natural parity exchange (probably $\rho$ or $f_{2}$ exchange), the $0^{++}$scalar state $a_{0 R}$ cannot be produced. Although the $2^{++} a_{2 R}$ can be produced (note the large $\rho \pi$ coupling), it has a weak coupling to the $\pi f_{1}$ final state and hence is not readily observable in this channel.

There is some very recent evidence for a $2^{3} \mathrm{P}_{2}$ state from the Crystal Barrel, who report an $a_{2}(1650)$ in $\eta \pi^{o}$ final states in $p \bar{p} \rightarrow \eta \eta \pi^{o}$ [30]. Although we expect $\eta \pi$ to be a relatively minor mode, with a branching fraction of $7 \%$, the mass and reported width of $\Gamma=260(15) \mathrm{MeV}$ are consistent with expectations (Table B5). The final states $\rho \pi$ and $\rho \omega$ are predicted to have large couplings to an $a_{2 R}$ state, so we expect a large signal in these $3 \pi$ and $5 \pi$ final states.

The prediction of a large coupling to vector meson pairs suggests $\gamma \gamma \rightarrow 2{ }^{3} \mathrm{P}_{J} \rightarrow V V$ as a possible source of the $a_{0 R}$ and $a_{2 R}$ states. Indeed, ARGUS has evidence that the $\rho \omega$ final state near threshold is mainly in the partial wave $\mathrm{J}^{P C}=2^{++}, \mathrm{J}_{z}=2$, and the $\gamma \gamma \rightarrow \rho^{o} \omega$ 
cross section is at maximum near $1.7 \mathrm{GeV}$ [31]. The $\mathrm{J}_{z}=2$ signal is characteristic of a $2^{++}$resonance, as there is a selection rule [32] that $\gamma \gamma \rightarrow\left(J=2^{++}, \lambda=0\right)=0$ in the nonrelativistic quark model; hence $\lambda=2$ dominates. A study of $\gamma \gamma \rightarrow 5 \pi$ with improved statistics, perhaps at LEP2, may help to isolate these states. Of course the interpretation of any $\gamma \gamma \rightarrow V V$ reaction should be regarded as tentative until the large $\gamma \gamma \rightarrow \rho^{o} \rho^{o}$ signal [33] is understood, as this reaction also is dominated by $\mathrm{J}^{P C}=2^{++}, \mathrm{J}_{z}=2$, but contains both $\mathrm{I}=0$ and $\mathrm{I}=2$ projections in s-channel and hence cannot come from a single $q \bar{q}$ resonance. Finally, the reaction $\gamma \gamma \rightarrow a_{0 R} \rightarrow \pi b_{1}$ may also lead to a significant signal in $5 \pi$ final states, and could be isolated if the $\lambda=0$ selection rule is used to suppress the $a_{2 R}$ signal.

\section{C. $2^{++} 2^{3} \mathbf{P}_{2}: f_{2}(1600-1800)$}

Encouraged by the likely confirmation of the radial $1^{++} a_{1}(1700)$, we now turn our attention to the $2 \mathrm{P}$ isoscalar multiplet. First we consider the $f_{2}(1700) 2^{3} \mathrm{P}_{2} n \bar{n}$ radial tensor. We predict a large $\rho \rho$ width for the $2^{3} \mathrm{P}_{2} f_{2}(1700)$, and the modes $\omega \omega, \pi \pi$ and perhaps $\pi a_{2}$ should also be important (see Table B6). (Note that the simple branching fraction ratio $\rho \rho / \omega \omega \approx 3$ follows trivially from flavor counting.) The total width is predicted to be $\approx 400 \mathrm{MeV}$.

Although there is no strong evidence for such a state, there are suggestions of its presence in several processes. A large $2^{++}$enhancement referred to as the $X(1600)$, with $\Gamma=400(200) \mathrm{MeV}$, is well known in $\gamma \gamma \rightarrow \rho^{\circ} \rho^{o}[14,34]$. The small charged to neutral $\rho \rho$ ratio however precludes the identification of this signal with a single $f_{2}(1700)$ resonance. There are also reports of a rather narrow $f_{2}(1640)$ with a width of $\approx 60-120 \mathrm{MeV}$ in $\omega \omega[14,35-37]$. Although the predicted $2^{3} \mathrm{P}_{2} f_{2}(1700)$ width is much larger, it would be reduced somewhat by threshold effects in the $\omega \omega$ channel. Indeed, if the resonance mass is around $1700 \mathrm{MeV}$ and its width is several hundred $\mathrm{MeV}$, as suggested by our analysis, it may decay strongly into $\rho \rho$ (due to the large $\rho$ width leading to a favorable phase space), 
but the narrowness of the $\omega$ may cause only the upper part of the resonance to feed the $\omega \omega$ channel. Thus the resonance width in $\omega \omega$ may appear smaller than in $\rho \rho$, so both the $\mathrm{X}(1600)$ and the $f_{2}(1640)$ may be aspects of a single state.

A recent reanalysis of MarkIII data on $\psi \rightarrow \gamma \pi^{+} \pi^{+} \pi^{-} \pi^{-}$[27] similarly sees evidence of a $2^{++}$state near $\mathrm{M}=1.64 \mathrm{GeV}$, with $\Gamma=0.14 \mathrm{GeV}$, which couples strongly to $\rho \rho$. (In contrast they observe $0^{++}$states dominantly in $\sigma \sigma$.) This preference of the tensor state for $\rho \rho$ is consistent with ${ }^{3} \mathrm{P}_{0}$ model expectations for a $2^{3} \mathrm{P}_{2} f_{2}(1700)$ state (Table B6).

Finally, it is possible that the $f_{2}(1520)$ or "AX" state seen in $p \bar{p} \rightarrow 3 \pi$ [38] may be the low-mass tail of the $f_{2}(1700)$.

$$
\text { D. } 0^{++} 2^{3} \mathbf{P}_{0}: f_{0}(1500), f_{J}(1710)
$$

The $0^{++} f_{0}$ sector in the $1.5 \mathrm{GeV}$ mass region is clearly of interest for glueball searches. It is thus important to identify the ${ }^{3} \mathrm{P}_{0}$ quarkonia in this mass region. We stress that one should not be overly naive in this endeavor since strong recoupling effects, including couplings of quarkonia to nearby glueballs, are expected [28]. Nonetheless for initial theoretical guidance it will be useful to consider the predictions of the naive ${ }^{3} \mathrm{P}_{0}$ model for the decays of unmixed ${ }^{3} \mathrm{P}_{0} n \bar{n}$ quarkonia.

The decays predicted for the $2 \mathrm{P}$ scalar $f_{0}(1700)$ state in the ${ }^{3} \mathrm{P}_{0}$ model are given in Table B6. Fortunately they are very characteristic. The dominant modes are $\rho \pi \pi$, with approximately equal contributions from $\pi(1300) \pi$ and $a_{1}(1230) \pi$. The channels $\rho \rho$ and $\pi \pi$ are also important, and the total width is predicted to be $\approx 400 \mathrm{MeV}$. The $\eta \eta$ and KK amplitudes are both close to nodes and are predicted to be quite small.

The two well known scalar resonances in this mass region which can be compared to these predictions are the glueball candidate $f_{0}(1500)$ and the $f_{J}(1710)$. These states have PDG masses and total widths of $\mathrm{M}=1503(11) \mathrm{MeV}, \Gamma=120(19) \mathrm{MeV}$ and $\mathrm{M}=1697(4)$ $\mathrm{MeV}, \Gamma=175(9) \mathrm{MeV}$; both are rather narrow relative to expectations for a $2 \mathrm{P} n \bar{n}$ state. BES has recently reported [39] a spin parity analysis of the $\mathrm{K}^{+} \mathrm{K}^{-}$system in $\psi$ radiative 
decays; they see both $\mathrm{J}=0$ and $\mathrm{J}=2$ states. Both have widths of $\approx 100 \mathrm{MeV}$, much narrower than we expect for $2 \mathrm{P} n \bar{n}$ states. The presence of a significant $\eta \eta$ mode for both the $f_{0}(1500)$ and $f_{J}(1710)$ argues against a $2 \mathrm{P} n \bar{n}$ assignment. The possibility that a node in the $2 \mathrm{P}$ decay amplitude is consistent with the observed weakness of $f_{J}(1710) \rightarrow \pi \pi$ is found to be unrealistic in practice; although there are actually two nodes, the modes that are strongly suppressed by these in the ${ }^{3} \mathrm{P}_{0}$ model are $\eta \eta$ and $\mathrm{KK}$, not $\pi \pi$.

The disagreement of predicted decay modes of $2 \mathrm{P} n \bar{n}$ states with experiment for the $f_{0}(1500)$ and $f_{J}(1710)$ supports the suggestions that neither of these states is a quarkonium. Amsler and Close [28] have noted that the $f_{0}(1500)$ could be a glueball that is mixed with the nearby $n \bar{n}$ and $s \bar{s}$ basis states, which explains the observed branching fractions. Conversely, Weingarten [6] suggests that the $f_{J}(1710)$ is the scalar glueball, based on its mass and on lattice QCD evidence that flavor symmetry may be inaccurate in glueball decays, together with a different pattern of $q \bar{q} \leftrightarrow G$ mixing. It may be that the glueball, $n \bar{n}$ and $s \bar{s}$ basis states are all strongly mixed in this sector, so that an assumed separation into glueball and quarkonium states is inaccurate [40].

An alternative suggestion is that the $f_{J}(1710)$ may be a vector-vector molecule, analogous to the $f_{0}(980)$ and $a_{0}(980) K \bar{K}$ candidates. The two possibilities discussed in the literature are $\mathrm{K}^{*} \bar{K}^{*}[41]$ and $\mathrm{K}^{*} \bar{K}^{*}+\omega \phi[42]$; these both predict small nonstrange modes and large couplings to KK $\pi \pi$ final states. The weakness of the $\pi \pi$ mode is due to the presence of a hidden $s \bar{s}$ pair (just as for $f_{0}(980) \rightarrow \pi \pi$ ), since both models assume that the $f_{J}(1710)$ is dominantly $n s \bar{n} \bar{s}$ in flavor.

In any case the $2 \mathrm{P}$ scalar $n \bar{n}$ states (or resonances with large $2{ }^{3} \mathrm{P}_{0} n \bar{n}$ components) should appear in $\rho \pi \pi$ final states, so it would be useful to search for these states, especially in reactions that produce the $f_{0}(1500)$ or $f_{J}(1710)$.

Finally, we should consider the possibility that the $f_{J}(1710)$ is dominantly a $2^{3} \mathrm{P}_{2}$ $n \bar{n}$ tensor state (see Table B6), since the quantum numbers have not been determined definitively. Again the quarkonium assignment is inconsistent with experiment; the $\eta \eta$ 
coupling is predicted to be small, and $\pi \pi$ is predicted to be quite large. The largest mode, $\rho \rho$, has not been reported for the $f_{J}(1710)$. The total width of the $n \bar{n}$ state is again rather larger than reported for the $f_{J}(1710)$. One must conclude that the $f_{J}(1710)$ does not appear to be consistent with any $n \bar{n}$ quarkonium assignment.

$$
\text { E. } 1^{+-} 2^{1} \mathbf{P}_{1}: b_{1}(1700), h_{1}(1700)
$$

Predictions for the missing spin-singlet 2P states are given in Table B7. These are expected to be only about $250 \mathrm{MeV}$ wide, so they may be easy to detect. Reactions that produce the $h_{1}(1170)$ and $b_{1}(1231)$ are obviously the most promising for searches for their radial excitations. The $h_{1}(1700)$ couples dominantly to $\rho \pi$, so it may be observable for example in $\pi^{-} p \rightarrow \rho \pi n$, in production through natural-parity exchange. Its partner $b_{1}(1700)$ can be produced similarly in $\omega \pi$ final states, and less characteristically in $\rho \rho$.

\section{1D STATES:}

$$
\text { A. } 2^{-+}{ }^{1} \mathbf{D}_{2}
$$

Studies of the decays of hybrids in the flux tube model conclude that a $2^{-+}$member of the lowest hybrid multiplet may be observably narrow [2]. This hybrid multiplet is expected at $\approx 1.8-1.9 \mathrm{GeV}[3,4]$, which overlaps the Godfrey-Isgur quark model predictions of $1.68 \mathrm{GeV}$ for the ${ }^{1} \mathrm{D}_{2} n \bar{n}, 1.89 \mathrm{GeV}$ for ${ }^{1} \mathrm{D}_{2} s \bar{s}$, and $2.13 \mathrm{GeV}$ for $2{ }^{1} \mathrm{D}_{2} n \bar{n}$ [19]. Thus it may be necessary to use characteristic branching fractions to distinguish quarkonia from hybrids in this mass region. Of course the $\pi_{2}(1670)$ is presumably $n \bar{n}$ because it has well established 1D multiplet partners such as the $\rho_{3}(1691)$, but distinguishing the higher-mass $s \bar{s}$ and $2 \mathrm{D}$ quarkonia from hybrids may not be so straightforward. 
Experimentally, the $\pi_{2}(1670)$ couples most strongly to $f_{2}(1275) \pi(\approx 56 \%)$ and $\rho \pi$ $\left(\approx 31 \%\right.$ ), with weaker couplings (at the $5-10 \%$ level) to $f_{0}(1300) \pi$ and $K^{*} K$. The 1996 PDG total width is $258(18) \mathrm{MeV}$ [14]. In comparison, the ${ }^{3} \mathrm{P}_{0}$ model predicts a total width of $250 \mathrm{MeV}$, with branching fractions of $f_{2}(1275) \pi(\approx 30 \%), \rho \pi(\approx 47 \%)$ and $\mathrm{K}^{*} \mathrm{~K}$ $(\approx 12 \%)$; these are in reasonable qualitative agreement with experiment. There is however disagreement with experiment in that little $f_{0}(1300) \pi$ is expected; we predict a branching fraction of only $0.2 \%$ to this mode, whereas the PDG value is $8.7(3.4) \%$. The largest as yet unreported mode should be $\rho \omega$, predicted to have a branching fraction of $11 \%$.

In addition to the plausible quarkonium state $\pi_{2}(1670)$, the ACCMOR Collaboration in 1981 noted a $2^{-+}$structure near $1.8 \mathrm{GeV}$, coupled to $f_{2} \pi$ and weakly to $f_{0}(1300) \pi$ and $\rho \pi$ [43]. This is similar to reports of a possible $2^{-+}$(or even $1^{-+}$) seen in photoproduction of $3 \pi$ states near $1.77 \mathrm{GeV}$ with a width of $100-200 \mathrm{MeV}$, which couples to $\rho \pi$ and $f_{2} \pi$ [44]. The VES Collaboration also claims a peak near $1.8 \mathrm{GeV}$, which they believe however to be non-resonant [45]. Lastly, two-photon experiments which see the $\pi_{2}(1670)$ in $\gamma \gamma \rightarrow \pi_{2} \rightarrow \pi^{0} \pi^{0} \pi^{0}[46]$ and $\gamma \gamma \rightarrow \pi_{2} \rightarrow \pi^{+} \pi^{-} \pi^{0}[47]$ also see indications of a possible contribution around $1.8 \mathrm{GeV}$. (In both cases the data appear skewed towards the higher masses relative to simple Breit Wigner and PDG values.) This may be expected for $\pi_{2(D)}$ through VMD as its $\rho \omega$ coupling is predicted to be large and thereby provide a further probe for any $2 \mathrm{D}$ component in $\pi_{2}(1800)$ state. It may be possible for LEP2 to clarify this situation.

If there is indeed a second $\pi_{2}$ state near $1.8 \mathrm{GeV}$, it is much too light to be a radial excitation of the $\pi_{2}(1670)$, and may instead be a hybrid. To test this possibility we have calculated the branching fractions of a $\pi_{2}(1800)$ hybrid in the flux tube model, and for comparison we show the partial widths of a hypothetical 1D quarkonium $\pi_{2}(1800)$. These are given in Table V. (The partial widths to $a_{1}(1230) \eta$ and $\mathrm{K}_{1}^{*}(1273) \mathrm{K}$ are $<1 \mathrm{MeV}$ in both models, so these modes are not displayed.) 
TABLE V. Partial widths of $1 \mathrm{D}$ and hybrid $\pi_{2}(1800)$ states.

\begin{tabular}{lccccccccc}
\hline & $\rho \pi$ & $\omega \rho$ & $\rho_{R} \pi$ & $b_{1} \pi$ & $f_{0} \pi$ & $f_{1} \pi$ & $f_{2} \pi$ & $\mathrm{K} * \mathrm{~K}$ & total \\
\hline$\pi_{2(1 D)}(1800)$ & 162. & 69. & 0. & 0. & 1. & 5. & 86. & 49. & 372. \\
$\pi_{2(H)}(1800)$ & 8 & 0 & 5 & 15 & 1 & 0 & 50 & 1 & 80 \\
\hline \hline
\end{tabular}

Evidently there are very characteristic differences between hybrid and $1 \mathrm{D}\left(\pi_{2}\right)$ branching fractions. First, note that a large $f_{2}(1275) \pi$ mode is not distinctive; this is expected from both states. A $1 \mathrm{D}$ quarkonium should also couple strongly to $\rho \pi, \omega \rho$ and $\mathrm{K}^{*} \mathrm{~K}$, and the total width should be about $400 \mathrm{MeV}$. In contrast, these $\mathrm{S}+\mathrm{S}$ modes are weak for a hybrid; the second largest mode (after $f_{2} \pi$ ) should be $b_{1} \pi$, which is forbidden to quarkonium by the singlet selection rule. Clearly a study of $b_{1} \pi$ final states in processes that report a $\pi_{2}(1800)$ would be very useful as a hybrid search. Other modes are quite small, so the hybrid should be a relatively narrow state, with a total width of only about $100 \mathrm{MeV}$. In summary, the characteristic signature of a $\pi_{2(H)}(1800)$ hybrid is a strong $f_{2} \pi$ mode and some $b_{1} \pi$ but weak couplings to $\rho \pi, \omega \rho$ and $K^{*} \mathrm{~K}$.

\section{C. $\eta_{2}$}

A doubling of $2^{-+}$peaks has also been reported by Crystal Barrel, in the isoscalar sector in $p \bar{p} \rightarrow\left(\eta \pi^{o} \pi^{o}\right) \pi^{o}[48]$. Masses and widths of $\mathrm{M}=1645(14)(15) \mathrm{MeV}, \Gamma=$ $180_{-21}^{+40}(25) \mathrm{MeV}$ and $\mathrm{M}=1875(20)(35) \mathrm{MeV}, \Gamma=200(25)(45) \mathrm{MeV}$ have been reported for the two $2^{-+}$states. This $\eta_{2}(1645)$ is seen in $a_{2}(1318) \pi[49]$, and in view of the approximate degeneracy with the $\pi_{2}(1670)$ and other $1 \mathrm{D}$ candidates is probably the ${ }^{1} \mathrm{D}_{2}$ $n \bar{n}$ isosinglet partner of $\pi_{2}(1670)$. The higher-mass state $\eta_{2}(1875)$ has been seen only in $f_{2}(1275) \eta$ (only $50 \mathrm{MeV}$ above threshold), and no evidence of it is found in $a_{0}(980) \pi$, $f_{0}(980) \eta$ or $f_{0}(1300) \eta$. The Crystal Ball Collaboration some time ago reported a $2^{-+}$(or possibly $0^{-+}$) at $1880 \mathrm{MeV}$, with a width of $220 \mathrm{MeV}$, decaying equally to $a_{2}(1318) \pi$ and $a_{0}(980) \pi[46]$. These data are also consistent with a contribution from $\eta_{2}(1645)$. One 
TABLE VI. Partial widths of $1 \mathrm{D}$ and hybrid $\eta_{2}(1875)$ states.

\begin{tabular}{lcccccccc}
\hline \hline & $\rho \rho$ & $\omega \omega$ & $f_{2} \eta$ & $a_{0}(1450) \pi$ & $a_{1} \pi$ & $a_{2} \pi$ & $\mathrm{K}^{*} \mathrm{~K}$ & total \\
\hline$\eta_{2(1 D)}(1875)$ & 147. & 46. & 45. & 1. & 43. & 264. & 61. & 607. \\
$\eta_{2(H)}(1875)$ & 0 & 0 & 20 & 2 & 0 & 160 & 10 & $\approx 190$ \\
\hline \hline
\end{tabular}

expects $\gamma \gamma \rightarrow \eta_{2}>\gamma \gamma \rightarrow \pi_{2}$, with the magnitude of the signal in $\gamma \gamma \rightarrow \eta \pi \pi$ depending on $\operatorname{BR}\left(\eta_{2} \rightarrow \eta \pi \pi\right)$. Here again LEP2 may have much to contribute.

In Table VI we compare the decay modes expected for a hybrid at $1875 \mathrm{MeV}$ with ${ }^{3} \mathrm{P}_{0}$ model predictions for a hypothetical ${ }^{1} \mathrm{D}_{2} \eta_{2}$ (1875) quarkonium. Both assignments lead to a significant $f_{2} \eta$ signal, and both predict a much larger $a_{2} \pi$ mode.

The most characteristic modes are $\rho \rho$ and $\omega \omega$, which should be very weak for a hybrid but large for a 1D quarkonium. Similar results follow for $K^{*} K$ and $a_{1} \pi$. Clearly searches for $a_{2} \pi, \rho \rho$ and $\omega \omega$ would be most useful. The large predicted coupling to $\rho \rho$ for the $\eta_{2(1 D)}$ encourages a search in $\gamma \gamma$ for this state.

\section{D. ${ }^{3} \mathbf{D}_{J}$ states}

Here we consider only the ${ }^{3} \mathrm{D}_{3}$ and ${ }^{3} \mathrm{D}_{2}$ states since the ${ }^{3} \mathrm{D}_{1}$ vectors were previously discussed with the $2^{3} \mathrm{~S}_{1}$ states. The $3^{--}$states $\rho_{3}(1691)$ and $\omega_{3}(1667)$ are well established ${ }^{3} \mathrm{D}_{3} n \bar{n}$ quarkonia, with masses as expected for $1 \mathrm{D}$ states and widths of about $200 \mathrm{MeV}$. The $\rho_{3}$ (Table B7) is expected to decay mainly to $\rho \rho(41 \%)$ and $\pi \pi(34 \%)$, with a somewhat weaker $\omega \pi$ mode (11\%). Experimentally the decays to $4 \pi$ are about $70 \%$, of which $16(6) \%$ is $\omega \pi$. The $\pi \pi$ branching fraction is observed to be $23.6(1.6) \%$. There are also KK and $K^{*} K$ modes of a few percent, roughly as predicted. The total width is predicted to be $174 \mathrm{MeV}$ with these parameters, consistent with observation. Thus the $\rho_{3}(1691)$ appears to decay approximately as predicted by the ${ }^{3} \mathrm{P}_{0}$ model, which supports the application of the model to decays of high-L states.

Its isoscalar partner $\omega_{3}(1667)$ is a more interesting case. Since few modes are open 
and the couplings are rather weak, we predict a total width of only $69 \mathrm{MeV}$. Although this appears inconsistent with the PDG width of $168(10) \mathrm{MeV}$, this observed value is presumably broadened by the hadronic width of the $\rho$ and $b_{1}$ in the two-body modes $\rho \pi$ and $b_{1} \pi$. The reported modes are $\rho \pi$ and $\omega \pi \pi$; we expect $\rho \pi$ to be dominant, with $\approx 10 \%$ branches to $b_{1} \pi$ (the source of $\omega \pi \pi$ ?) and KK. The KK mode affords an opportunity to measure the actual width of the $\omega_{3}$, which may be much smaller than it appears in $\rho \pi$ and $b_{1} \pi$ modes.

Our results for the ${ }^{3} \mathrm{D}_{2} 2^{--}$states $\rho_{2}(1670)$ and $\omega_{2}(1670)$ are especially interesting because these are "missing mesons" in the quark model. We find that these are rather broad states, with total widths of about $300-400 \mathrm{MeV}$. The $\rho_{2}$ is predicted to have a large branching fraction of $54 \%$ to $a_{2} \pi$, so it should be observable in this final state or in the secondary modes $\omega \pi$ or $K^{*} K$. The $\omega_{2}$ is predicted to have an even larger branching fraction of $74 \%$ to $\rho \pi$. It too couples significantly to $\mathrm{K}^{*} \mathrm{~K}$, and may also be observable in $\omega \eta$

\section{1F STATES}

The $1 \mathrm{~F}$ states provide us with an opportunity to test the accuracy of the ${ }^{3} \mathrm{P}_{0}$ decay model predictions for higher quarkonium states, since the $4^{++}$and $3^{+ \pm}$states expected near $2.05 \mathrm{GeV}$ do not have competing assignments as glueballs or hybrids. At present only two of these states are reasonably well established, the $f_{4}(2044)$ and $a_{4}(2037)$ [14]. There is also some evidence for an $a_{3}(2080)$ [16].

We do not yet have experimental branching fractions for the $I=11 \mathrm{~F}$ states. The $a_{4}(2037)$ is seen in $\mathrm{KK}$ and $3 \pi$, and the $a_{3}(2080)$ is reported in $3 \pi$ and $\rho_{3}(1691) \pi$, with $\rho_{3} \pi$ dominant. The branching fractions of the $f_{4}(2044)$ are known with more accuracy; $\omega \omega$ and $\pi \pi$ are important modes, $26(6) \%$ and $17.0(1.5) \%$. KK and $\eta \eta$ modes are both known, with reported branching fractions of about $0.7 \%$ and $0.2 \%$ respectively.

${ }^{3} \mathrm{P}_{0}$ predictions for the decays of these ${ }^{3} \mathrm{~F}_{J}$ states are given in Tables B11 and B12. 
The $a_{4}(2050)$ is indeed expected to appear in $3 \pi$ (mainly $\rho \pi$ ), and the dominant mode is predicted to be $\rho \omega$. This state is predicted to be rather narrower than reported. The $a_{3}(2080)$ is predicted to decay dominantly to $\rho_{3} \pi$, as is observed. The $3 \pi$ mode is also predicted to be large, and to arise from both $\rho \pi$ and $f_{2} \pi$. The $f_{4}(2044){ }^{3} \mathrm{P}_{0}$ model predictions are also in qualitative agreement with experiment, in that $\pi \pi$ and $\omega \omega$ are expected to be important modes, as observed. The $f_{4}$ partial widths to pseudoscalar pairs are uniformly too large, for example $\Gamma_{f_{4} \rightarrow \pi \pi}^{\text {thy. }}=62 . \mathrm{MeV}$ but $\Gamma_{f_{4} \rightarrow \pi \pi}^{\text {expt. }}=35(4) \mathrm{MeV}$. This decay however is $\mathrm{G}$-wave, so the rate has a prefactor of $\left|\vec{p}_{\pi} / \beta\right|^{9}$; this extreme sensitivity means that a small increase of $\beta$ by $\approx 10 \%$, halves the decay rate and gives agreement with experiment. Thus this disagreement is quite sensitive to parameters and is probably not significant.

The predictions for branching fractions of the five missing $I=0,11 \mathrm{~F}$ states suggest that several of them may easily be found by reconstructing the appropriate final states. The total widths of all except the ${ }^{3} \mathrm{~F}_{2}$ states are predicted to be $\sim 300 \mathrm{MeV}$, so they should be observable experimentally. The $f_{3}(2050)$ is predicted to couple dominantly to $a_{2} \pi$. In the spin-singlet ${ }^{1} \mathrm{~F}_{3}$ sector, the $h_{3}(2050)$ should appear in $\rho \pi$ and $\rho_{3}(1691) \pi$, just as we found for the $a_{3}(2080)$. The $b_{3}(2050)$ should be evident in $a_{2} \pi$, and less strongly in $\omega_{3} \pi, \omega \pi$ and $\rho \rho$. Modes such as $a_{2} \pi$ are preferable because the two-body mesons are not excessively broad and they are far from threshold, so a resonance can be distinguished from a threshold effect. In some cases the amplitude structure of these final states is also characteristic; these can be determined from the results quoted in App.A.

The missing ${ }^{3} \mathrm{~F}_{2}$ states may be more difficult to identify, as we predict large total widths of $\approx 600 \mathrm{MeV}$ for these states. The $a_{2}(2050)$ couples most strongly to $b_{1} \pi ; \eta_{2}(1645) \pi$ and $K_{1}^{*}(1273) K$ are other important modes. Its $\mathrm{I}=0$ partner $f_{2}(2050)$ should be evident in $\pi_{2}(1670) \pi$ and will also populate $K_{1}^{*}(1273) K$ final states.

Identification of these $1 \mathrm{~F}$ states and determination of their branching fractions and decay amplitudes will be a very useful contribution to the study of resonances, as it 
will allow detailed tests of the usefulness of the ${ }^{3} \mathrm{P}_{0}$ model as a means for identifying quarkonium states in this crucial $2 \mathrm{GeV}$ region.

\section{SUMMARY AND EXPERIMENTAL STRATEGY}

We have established that the $a_{1}(1700)$ is very likely a $2 \mathrm{P}$ radial excitation. This follows from the weak S-wave and strong $\mathrm{D}$-wave in $\rho \pi$. This also establishes the natural mass scale for the $2 \mathrm{P}$ multiplets as $\approx 1.7 \mathrm{GeV}$. We have been unable to identify radial scalars. These are predicted to be broad, and so their non-appearance is not surprising. Conversely it raises interest in the (relatively narrow) $f_{0}(1500)$ and possible scalar $f_{J}(1710)$. We do identify some (more speculative) potential candidates for $2^{++} 2 \mathrm{P}$ members. We note that $\gamma \gamma$ production may help identify these radial $2 \mathrm{P}$ states and also clarify the nature of $f_{0}(1500)$ and $f_{J}(1710)[40]$.

The $\pi(1300)$ and $\eta(1295)$ appear to be convincing $2 S$ states. This conclusion is based on their relative widths; the large $\rho \pi$ mode of the $\pi(1300)$ has no analog for its $\eta$ counterparts. The status of the $\eta(1440)$ remains open; the mass and width suggest a dominantly $s \bar{s}$ state, but the $\gamma \rho$ mode argues against it. Studies of $\psi \rightarrow \eta(1295,1440)+(\omega, \phi)$ and $\psi \rightarrow \gamma+(\gamma \omega, \gamma \rho, \gamma \phi)$ may identify the flavor content of these $\eta$ states.

The $\rho(1465)$ and $\omega(1419)$ have masses that are consistent with radial $2 \mathrm{~S}$ but their decays show characteristics of hybrids, as noted previously [2]. We suggest that these states may be 2S-hybrid mixtures analogous to the 3S-hybrid mixing suggested for the $c \bar{c}[50]$. This can be tested by accurate measurement of the partial widths of these states and their vector partners at $1.6-1.7 \mathrm{GeV}$ to $\pi \pi, \omega \pi$, and especially $h_{1} \pi$ and $a_{1} \pi$.

The $3 \mathrm{~S} \pi$ is expected in the $1800 \mathrm{MeV}$ mass region as is a $\pi_{H}$ hybrid. We find that the decay patterns of these states are very different. A strong $f_{0}(1300) \pi$ from the hybrid contrasted with a large $\rho \omega$ mode from the $3 \mathrm{~S}$ quarkonium is the sharpest discriminant. The VES state $\pi(1800)$ clearly exhibits this hybrid signature. It is now necessary to establish the presence of $0^{-+}$in the $\rho \omega$ channel, and to see if any resonant state is present 
that is distinct from the $\pi(1800)$ seen in $f_{0}(1300) \pi$. It is possible that there are two $\pi(\approx 1800)$ states, $q \bar{q}$ and hybrid, whose production mechanisms and decay fractions differ sufficiently so that they can be separated. We suggest that the possibility of two such $\pi(\approx 1800)$ states be allowed for in data analyses.

In the immediate future there are opportunities for $\gamma \gamma$ physics at LEP2 and at B factories. Possible strategies for isolating some of these higher quarkonia include:

- $\gamma \gamma \rightarrow 5 \pi$ contains (i) $\rho \omega$ which may access the radial $a_{0 R}$ and $a_{2 R}$ near $1700 \mathrm{MeV}$ and a possible $\pi_{3 S}(1800)$. (ii) $\pi b_{1}$ which can isolate the $a_{0 R}$ if the helicity selection rule [32] is used to suppress the $a_{2 R}$.

- $\gamma \gamma \rightarrow 4 \pi$ may access the radial $f_{2 R}$ near $1700 \mathrm{MeV}$ through its decay into $\rho \rho$. The $4 \pi$ channel may also be searched for the $f_{0}(1500)$ since this state is known to have a significant branching fraction to $4 \pi$ but should have a suppressed $\gamma \gamma$ coupling if it is a glueball [40].

- $\gamma \gamma \rightarrow 3 \pi$ may be searched for $2^{-+}$states in order to verify whether the established $\pi_{2}(1670)$ is accompanied by a higher $\pi_{2}(1800)$ in $3 \pi^{\circ}$ and $\pi^{+} \pi^{-} \pi^{o}$. This $3 \pi$ system may also be studied for evidence of one or more $\pi(1800)$ states.

- $\gamma \gamma \rightarrow \eta \pi \pi$ may access the isoscalar partners of these $\pi_{2}$ states.

In the near future it will be possible to study $e^{+} e^{-}$annihilation up to $\approx 2 \mathrm{GeV}$ at DAFNE. The channels $e^{+} e^{-} \rightarrow 4 \pi$ should be measured and $\pi a_{1}$ and $\pi h_{1}$ states separated in order to carry out the analysis of hybrid and radial vector components in section 3B. The isoscalar partners of the vectors also need confirmation, and final states with kaons are needed to investigate possible $\omega-\phi$ mixing; a potential weakness of the present data analyses is that such flavor mixing is assumed to be unimportant.

In the next century there will be new opportunities at the COMPASS facility at CERN. This will enable further studies of central production and also of diffractive excitation. For the latter one may anticipate improved studies of the $\pi$ excitations (such as the $\pi(1300)$ and $\pi(1800)$ states), possibly including Primakoff excitation. Judicious studies of specific 
final states as discussed above may help separate $3 \mathrm{~S}$ and hybrid states. The use of $\mathrm{K}$ beams will allow analogous studies of the strange counterparts of these states and may help to clarify the spectrum of quarkonia, glueballs and hybrids.

Experiments with $\pi$ beams can access the following interesting channels.

- $\pi p \rightarrow\left(\pi f_{1}\right) p$, to confirm the D-wave dominance of $a_{1 R}(1700)$ and to seek its partner $a_{2 R}$.

- $\pi p \rightarrow\left(\pi f_{2}\right) p$ can access both $\pi_{2(1 D)}$ and $\pi_{2(H)}$. These can be separated in $b_{1} \pi$; the singlet selection rule forbids this mode for $\pi_{2(1 D)}$ but allows it for $\pi_{2(H)}$. $(\pi \rho) p$ can also separate $\pi_{2(1 D)}$ from $\pi_{2(H)} ; \pi_{2(1 D)} \rightarrow \rho \pi$ is the dominant mode whereas $\pi_{2(H)}$ is much suppressed into $\mathrm{S}+\mathrm{S}$ hadrons.

- $(\pi \pi),(\pi \omega),\left(a_{1} \pi\right)$ and $\left(h_{1} \pi\right)$ are important in the interpretation of the vectors between 1.4 and $1.7 \mathrm{GeV}$, which may contain large hybrid components.

- $\left(f_{0} \pi\right),\left(f_{2} \pi\right)$ and $(\rho \omega)$ can all be searched for evidence of $\pi(1800)$ states.

- $\pi^{-} p \rightarrow(\pi \rho)^{o} n$ or $(\pi \omega)^{o} n$ access respectively $h_{1 R}$ and $b_{1 R}$.

Finally, many two-body channels are predicted to couple strongly to specific $2 \mathrm{P}, 1 \mathrm{D}$ and $1 \mathrm{~F}$ states, as shown in Appendix B. These include "missing mesons" such as the ${ }^{3} \mathrm{~F}_{2}$ and most $2 \mathrm{P}$ states, and studies of these two-body final states may reveal the missing resonances. The modes $a_{2} \pi, \rho \rho$ and $b_{1} \pi$ are important for many of these missing states and merit careful investigation.

We reiterate that it is in general a good strategy to study decays into both $\mathrm{S}+\mathrm{S}$ and $\mathrm{S}+\mathrm{P}$ meson modes, as the relative couplings of these modes are usually quite distinct for hybrid versus quarkonium assignments. 


\section{ACKNOWLEDGMENTS}

We would like to acknowledge useful communications with C.Amsler, D.V.Bugg, S.U.Chung, G.Condo, K.Danyo, A.Dzierba, S.Godfrey, I.Kachaev, Y.Khokhlov, A.Kirk, D.Ryabchikov and A.Zaitsev. This work was supported in part by the United States Department of Energy under contracts DE-FG02-96ER40944 at North Carolina State University and DE-AC05-96OR22464 managed by Lockheed Martin Energy Research Corp. at Oak Ridge National Laboratory. FEC is supported in part by European Community Human Capital Mobility Programme Eurodafne, Contract CHRX-CT92-0026. 
[1] N.Isgur, R.Kokoski and J.Paton, Phys. Rev. Lett. 54, 869 (1985).

[2] F.E.Close and P.R.Page, Nucl. Phys. B443, 233 (1995); Phys. Rev. D52, 1706 (1995).

[3] N.Isgur and J.Paton, Phys. Rev. D31, 2910 (1985).

[4] T.Barnes, F.E.Close and E.S.Swanson, Phys. Rev. D52, 5242 (1995); see also C.Michael [5].

[5] G.Bali et al. (UKQCD Collaboration), Phys. Lett. B309, 378 (1993); D.Weingarten, Nucl. Phys. B (Proc. Suppl.) 34, 29 (1994); C.Michael, Liverpool report LTH 370, hep-ph/9605243 (May 1996); F.E.Close and M.J.Teper, "On the lightest Scalar Glueball", RAL-96-040 / OUTP-96-35P (July 1996).

[6] J.Sexton, A.Vaccarino and D.Weingarten, Phys. Rev. Lett. 75, 4563 (1995).

[7] D.V.Amelin et al. (VES Collaboration), Phys. Lett. B356 (1995) 595.

[8] S.U.Chung, private communication.

[9] F.E.Close, p.1395 in Proc. XXVII International Conf. on High Energy Physics, Glasgow, (Institute of Physics, U.K., 1994, P.Bussey and I.Knowles eds.).

[10] A.M.Zaitsev (VES Collaboration), p.1409 in Proc. XXVII International Conf. on High Energy Physics, Glasgow, (Institute of Physics, U.K., 1994, P.Bussey and I.Knowles eds.).

[11] E.S.Ackleh, T.Barnes and E.S.Swanson, "On the Mechanism of Open-Flavor Strong Decays", ORNL-CTP-96-03, hep-ph-9604355 (April 1996), Phys. Rev. D (to appear).

[12] A.LeYaouanc, L.Oliver, O.Pène and J.Raynal, Phys. Rev. D8, 2223 (1973); see also ibid., D9, 1415 (1974); D11, 1272 (1975); L.Micu, Nucl. Phys. B10, 521 (1969).

[13] G.Busetto and L.Oliver, Z.Phys. C20, 247 (1983); R.Kokoski and N.Isgur, Phys. Rev. D35, 907 (1987); P.Geiger and E.S.Swanson, Phys. Rev. D50, 6855 (1994); H.G.Blundell and 
S.Godfrey, Phys. Rev. D53, 3700 (1996).

[14] Particle Data Group, Phys. Rev. D54, 1 (1996).

[15] G.Bellini et al., Phys. Rev. Lett. 48, 1697 (1982).

[16] Particle Data Group, Phys. Rev. D50, 1173 (1994).

[17] Yu. Prokoshkin (GAMS Collaboration), in Proc. of LEAP96, Dinkelsbühl, Germany, 27-31 August 1996.

[18] A.B.Clegg and A.Donnachie, Z. Phys. C62, 455 (1994).

[19] S.Godfrey and N.Isgur, Phys. Rev. D32, 189 (1985).

[20] A.Donnachie and Yu.S.Kalashnikova, Z.Phys. C59, 621 (1993).

[21] A.Abele et al. (Crystal Barrel Collaboration), "High-mass $\rho$-meson states from $\bar{p} d$ annihilation at rest into $\pi^{-} \pi^{o} \pi^{o} p_{\text {spectator }}$, Phys. Lett. B (to appear).

[22] VES Collaboration, "Diffractive reaction $\pi^{-} A \rightarrow \eta \eta \pi^{-} A$ study at $37 \mathrm{GeV} / \mathrm{c}^{\prime}$. (unpublished)

[23] T.Barnes and E.S.Swanson, in preparation.

[24] Y.Khokhlov, private communication

[25] R.M.Baltrusaitis et al. (MarkIII Collaboration) Phys. Rev. Lett. 55, 1723 (1985); Phys. Rev. D33, 1222 (1986).

[26] D.Bisello et al.. (DM2 Collaboration) Phys. Lett. B192, 239 (1987); Phys. Rev. D39, 701 (1989).

[27] D.V.Bugg et al., Phys. Lett. B353, 378 (1995).

[28] C.Amsler and F.E.Close, Phys. Lett. B353, 385 (1995); Phys. Rev. D53, 295 (1996).

[29] J.H.Lee et al., Phys. Lett. B323, 227 (1994).

[30] T.Degener (Crystal Barrel Collaboration), in Proc. of LEAP96, Dinkelsbühl, Germany, 27- 
31 August 1996.

[31] G.Kernel (ARGUS), Proc. of PHOTON95 (World Scientific, 1995, eds. D.J.Miller, S.L.Cartwright and V.Khoze), pp.226-231, esp. Fig.2; E.Križnič, Doctoral thesis, University of Ljubljana (1993); E.Križnič, Proc. XXVII International Conf. on High Energy Physics, Glasgow 1994, (Institute of Physics, U.K., 1994, P.Bussey and I.Knowles eds.), p.1413.

[32] Z.P.Li, F.E.Close and T.Barnes, Phys. Rev. D43, 2161 (1991); E.S.Ackleh, T.Barnes and F.E.Close, Phys. Rev. D46, 2257 (1992); T.Barnes, in Proc. IXth International Workshop on Photon-Photon Collisions, La Jolla, CA 22-26 March 1992 (World Scientific, 1992, D.O.Caldwell and H.P.Paar eds.).

[33] See D.Morgan, M.R.Pennington and M.R.Whalley, J. Phys. G20, A1 (1994) for a review of $\gamma \gamma \rightarrow V V$ data.

[34] H.Albrecht et al., Zeit. Phys. C50, 1 (1991).

[35] H.Albrecht et al. (ARGUS Collaboration), Phys. Lett. B374, 265 (1996).

[36] G. M. Beladidze et al., Zeit. Phys. C54, 367 (1992).

[37] A. Adamo et al., Phys. Lett. B287, 368 (1992).

[38] B.May et al. (ASTERIX Collaboration), Phys. Lett. B225, 450 (1989); E.Aker et al. (Crystal Barrel Collaboration), Phys. Lett. B260, 249 (1991).

[39] J.Bai et al (BES Collaboration) "The Structure Analysis of the $f_{J}(1710)$ in the Radiative Decay $\psi \rightarrow \gamma K^{+} K^{-}$, IHEP Report (July 1996).

[40] F.E.Close, G.Farrar and Z.P.Li, "Determining the Gluonic Content of Isoscalar Mesons", RAL-96-052.

[41] N.A.Törnqvist, Phys. Rev. Lett. 67, 556 (1991).

[42] K.Dooley, E.S.Swanson and T.Barnes, Phys. Lett. B275, 478 (1992). 
[43] C.Daum et al., Nucl. Phys. B182, 269 (1981).

[44] G.Condo et al., Phys. Rev. D43, 2787 (1991). See also Y.Eisenberg et al., Phys. Rev. Lett. 23, 1322 (1969); D.Aston et al., Nucl. Phys. B189, 15 (1981).

[45] D.I.Ryabchikov (VES Collaboration), Proc. of Hadron95, Manchester, U.K., Aug. 1995.

[46] D.Antreasyan et al. (Crystal Ball Collaboration), Z.Phys. C48, 561 (1990).

[47] H.J.Behrend et al. (Cello Collaboration), Z.Phys. C46, 583 (1990).

[48] D.V.Bugg et al., Z.Phys. C (to appear), "Study of $p \bar{p} \rightarrow \eta \pi^{0} \pi^{0} \pi^{0}$ at $1200 \mathrm{MeV} / \mathrm{c}$ ".

[49] C.Amsler et al. (Crystal Barrel Collaboration), Z. Phys. C71, 227 (1996).

[50] F.E.Close and P.R.Page, Physics Letters B366, 323 (1996). 


\section{APPENDIX A: A COMPILATION OF ${ }^{3} \mathbf{P}_{0}$ MODEL DECAY AMPLITUDES.}

We quote results for the ${ }^{3} \mathrm{P}_{0}$ model $\mathrm{A} \rightarrow \mathrm{BC}$ meson decay amplitudes in terms of an invariant amplitude $\mathcal{M}_{L_{B C} S_{B C}}$, which is the $\mathrm{L}_{B C} \mathrm{~S}_{B C}$ projection of the ${ }^{3} \mathrm{P}_{0}$ pair creation Hamiltonian matrix element divided by a momentum conserving delta function,

$$
\mathcal{M}_{L_{B C} S_{B C}}^{A \rightarrow B C}=\left\langle J_{A}, L_{B C}, S_{B C} \mid B C\right\rangle\left\langle B C\left|H_{I}\left({ }^{3} P_{0}\right)\right| A\right\rangle / \delta(\vec{A}-\vec{B}-\vec{C})
$$

This amplitude and the derivation of the ${ }^{3} \mathrm{P}_{0}$ matrix elements are discussed in detail in Appendix A of Ackleh et al. [11]. The partial widths $\Gamma_{A \rightarrow B C}$ are related to these decay amplitudes by

$$
\Gamma_{A \rightarrow B C}=2 \pi \frac{P E_{B} E_{C}}{M_{A}} \sum_{L S}\left|\mathcal{M}_{L S}\right|^{2}
$$

The full ${ }^{3} \mathrm{P}_{0}$ decay amplitude is the sum of two Feynman diagrams, called $d_{1}$ and $d_{2}$ (Fig.A1).

In a specified flavor channel these diagrams have flavor weight factors that multiply the spin-space matrix element. The flavor factors for all the processes considered in this paper are given in Table A1. The $\mathcal{M}$ amplitudes listed below are for unit flavor factors, $I_{\text {flavor }}\left(d_{1}\right)=+1$ and $I_{\text {flavor }}\left(d_{2}\right)= \pm 1$, with the phase chosen so they add rather than cancel. (The cancelations are due to flavor symmetries such as G-parity.) Thus for a physical decay such as $\rho^{+} \rightarrow \pi^{+} \pi^{o}$ one should multiply the unit-flavor amplitude $\mathcal{M}$ in $\mathrm{A} 3$ by $+1 / \sqrt{2}$ before computing the decay width using (A2). Some states populate several decay channels, for example $f \rightarrow \pi^{o} \pi^{o}$ as well as $\rightarrow \pi^{+} \pi^{-}$; to sum over all channels one should multiply the width by the flavor multiplicity factor $\mathcal{F}$ in the Table. In these flavor weights the pairs $(\pi, a),(\rho, b),(\omega, h)$ and $\left(f, \eta_{n \bar{n}}\right)$ are equivalent, up to factors due to identical particles in the final state. 

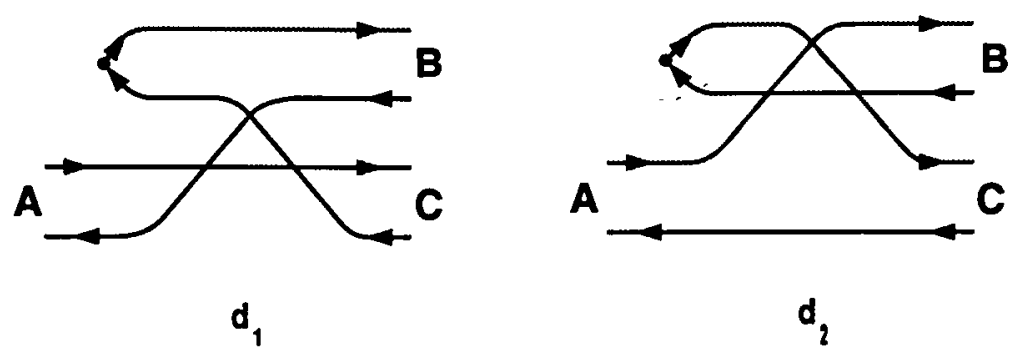

Figure A1. $q \bar{q}$ meson decay diagrams in the ${ }^{3} \mathrm{P}_{0}$ decay model.

\begin{tabular}{||c|c|c|c|c||}
\hline \multicolumn{5}{||c||}{ Table A1. Flavor Weight Factors. } \\
\hline Generic Decay & Subprocess & $I_{\text {flavor }}\left(d_{1}\right)$ & $I_{\text {flavor }}\left(d_{2}\right)$ & $\mathcal{F}$ \\
\hline$\rho \rightarrow \pi \pi$ & $\rho^{+} \rightarrow \pi^{+} \pi^{\circ}$ & $+1 / \sqrt{2}$ & $-1 / \sqrt{2}$ & 1 \\
\hline$f \rightarrow \pi \pi$ & $f \rightarrow \pi^{+} \pi^{-}$ & $-1 / \sqrt{2}$ & $-1 / \sqrt{2}$ & $3 / 2$ \\
\hline$f \rightarrow K K$ & $f \rightarrow K^{+} K^{-}$ & 0 & $-1 / \sqrt{2}$ & 2 \\
\hline$a \rightarrow \rho \pi$ & $a^{+} \rightarrow \rho^{+} \pi^{o}$ & $+1 / \sqrt{2}$ & $-1 / \sqrt{2}$ & 2 \\
\hline$a \rightarrow K K$ & $a^{+} \rightarrow K^{+} K^{o}$ & 0 & -1 & 1 \\
\hline$b \rightarrow \omega \pi$ & $b^{+} \rightarrow \omega \pi^{+}$ & $+1 / \sqrt{2}$ & $+1 / \sqrt{2}$ & 1 \\
\hline$h \rightarrow \rho \pi$ & $h \rightarrow \rho^{+} \pi^{-}$ & $-1 / \sqrt{2}$ & $-1 / \sqrt{2}$ & 3 \\
\hline$K^{*} \rightarrow K \pi$ & $K^{*+} \rightarrow K^{+} \pi^{o}$ & $+1 / \sqrt{2}$ & 0 & 3 \\
\hline$\phi \rightarrow K K$ & $\phi \rightarrow K^{+} K^{-}$ & +1 & 0 & 2 \\
\hline
\end{tabular}

We take all spatial wavefunctions to be SHO forms with the same width parameter $\beta$; as a result the $\mathcal{M}_{L S}$ decay amplitudes are proportional to an overall Gaussian in $x=P / \beta$ times a channel-dependent polynomial $\mathcal{P}_{L S}(x)$,

$$
\mathcal{M}_{L S}=\frac{\gamma}{\pi^{1 / 4} \beta^{1 / 2}} \mathcal{P}_{L S}(x) e^{-x^{2} / 12}
$$

where $\gamma$ is the ${ }^{3} \mathrm{P}_{0}$ pair production coupling constant [11]. To specify these amplitudes it suffices to quote the polynomial $\mathcal{P}_{L S}(x)$ for each decay channel. The complete set of ${ }^{3} \mathrm{P}_{0}$ decay amplitudes for all $q \bar{q}$ resonances with "excitation level" $\mathcal{N}_{A}=\mathrm{N}_{A}+\mathrm{L}_{A} \leq 4$ decaying 
into final states with $\mathcal{N}_{B} \leq \mathcal{N}_{A}-1$ and $\mathrm{C}={ }^{1} \mathrm{~S}_{0}$ (and $\mathrm{C}={ }^{3} \mathrm{~S}_{1}$ in most cases) is given below. For the relatively obscure transitions $3 \mathrm{~S} \rightarrow 1 \mathrm{D}+\mathrm{C}, 1 \mathrm{~F} \rightarrow 1 \mathrm{P}+\mathrm{C}, 1 \mathrm{~F} \rightarrow 2 \mathrm{P}+$ $\mathrm{C}$ and $1 \mathrm{~F} \rightarrow 1 \mathrm{D}+\mathrm{C}$ we restrict $\mathrm{C}$ to ${ }^{1} \mathrm{~S}_{0}$; this does not exclude any decays allowed by phase space.

We include a few additional amplitudes in this list. Some of these are of interest as couplings to virtual two-body states, although phase space nominally forbids the decay. 
$1 S \rightarrow 1 S+1 S$

$$
f_{P}=-\frac{2^{5}}{3^{3}} x
$$

${ }^{3} \mathrm{~S}_{1}$

$$
\begin{aligned}
\mathcal{P}_{10}^{\left({ }^{3} S_{1} \rightarrow{ }^{1} S_{0}+{ }^{1} S_{0}\right)} & =f_{P}{ }^{1} P_{1} \\
\mathcal{P}_{11}^{\left({ }^{3} S_{1} \rightarrow{ }^{3} S_{1}+{ }^{1} S_{0}\right)} & =-\sqrt{2} f_{P}{ }^{3} P_{1} \\
\mathcal{P}_{L S}^{\left({ }^{3} S_{1} \rightarrow{ }^{3} S_{1}+{ }^{3} S_{1}\right)} & = \begin{cases}\sqrt{\frac{1}{3}} f_{P} & { }^{1} P_{1} \\
0 & { }^{3} P_{1} \\
-\sqrt{\frac{20}{3}} f_{P} & { }^{5} P_{1} \\
0 & { }^{5} F_{1}\end{cases}
\end{aligned}
$$

${ }^{1} \mathrm{~S}_{0}$

$$
\begin{aligned}
& \mathcal{P}_{L S}^{\left({ }^{1} S_{0 \rightarrow{ }^{1}} S_{0}+{ }^{1} S_{0}\right)}=0 \\
& \mathcal{P}_{11}^{\left({ }^{1} S_{0} \rightarrow{ }^{3} S_{1}+{ }^{1} S_{0}\right)}=-\sqrt{3} f_{P}{ }^{3} P_{1} \\
& \mathcal{P}_{11}^{\left({ }^{1} S_{0} \rightarrow{ }^{3} S_{1}+{ }^{3} S_{1}\right)}=\sqrt{6} f_{P}{ }^{3} P_{1}
\end{aligned}
$$


$2 S \rightarrow 1 S+1 S$

(See $1 S \rightarrow 1 S+1 S$ for channel coefficients.)

$$
f_{P}=-\frac{2^{9 / 2} 5}{3^{9 / 2}} x\left(1-\frac{2}{15} x^{2}\right)
$$

$2 S \rightarrow 1 P+1 S$

$$
\begin{aligned}
& f_{S}=\frac{2^{4}}{3^{4}}\left(1-\frac{7}{9} x^{2}+\frac{2}{27} x^{4}\right) \\
& f_{D}=\frac{2^{9 / 2}(13)}{3^{6}} x^{2}\left(1-\frac{2}{39} x^{2}\right)
\end{aligned}
$$

$2^{3} \mathbf{S}_{1}$

$$
\begin{aligned}
& \mathcal{P}_{L S}^{\left(2^{3} S_{1} \rightarrow{ }^{1} P_{1}+{ }^{1} S_{0}\right)}= \begin{cases}f_{S} & { }^{3} S_{1} \\
f_{D} & { }^{3} D_{1}\end{cases} \\
& \mathcal{P}_{L S}^{\left(2^{3} S_{1} \rightarrow{ }^{3} P_{1}+{ }^{1} S_{0}\right)}=\left\{\begin{array}{cc}
-\sqrt{2} f_{S} & { }^{3} S_{1} \\
\sqrt{\frac{1}{2}} f_{D} & { }^{3} D_{1}
\end{array}\right. \\
& \mathcal{P}_{22}^{\left(2^{3} S_{1} \rightarrow{ }^{3} P_{2}+{ }^{1} S_{0}\right)}=-\sqrt{\frac{3}{2}} f_{D} \quad{ }^{5} D_{1} \\
& \mathcal{P}_{L S}^{\left(2^{3} S_{1} \rightarrow{ }^{1} P_{1}+{ }^{3} S_{1}\right)}=\left\{\begin{array}{cc}
-\sqrt{\frac{1}{2}} f_{D} & { }^{3} D_{1} \\
\sqrt{\frac{3}{2}} f_{D} & { }^{5} D_{1}
\end{array}\right. \\
& \mathcal{P}_{L S}^{\left(2^{3} S_{1} \rightarrow{ }^{3} P_{0}+{ }^{3} S_{1}\right)}=\left\{\begin{array}{cc}
-\sqrt{3} f_{S} & { }^{3} S_{1} \\
0 & { }^{3} D_{1}
\end{array}\right. \\
& \mathcal{P}_{L S}^{\left(2^{3} S_{1} \rightarrow{ }^{3} P_{1}+{ }^{3} S_{1}\right)}=\left\{\begin{array}{cc}
-2 f_{S} & { }^{3} S_{1} \\
-\frac{1}{2} f_{D} & { }^{3} D_{1} \\
\sqrt{\frac{3}{4}} f_{D} & { }^{5} D_{1}
\end{array}\right.
\end{aligned}
$$




$$
\mathcal{P}_{L S}^{\left(2^{3} S_{1} \rightarrow{ }^{3} P_{2}+{ }^{3} S_{1}\right)}=\left\{\begin{array}{cc}
0 & { }^{3} S_{1} \\
\sqrt{\frac{3}{20}} f_{D} & { }^{3} D_{1} \\
\frac{1}{2} f_{D} & { }^{5} D_{1} \\
-\sqrt{\frac{28}{5}} f_{D} & { }^{7} D_{1} \\
0 & { }^{5} G_{1}
\end{array}\right.
$$

$2^{1} S_{0}$

$$
\begin{aligned}
& \mathcal{P}_{00}^{\left(2^{1} S_{0} \rightarrow{ }^{3} P_{0}+{ }^{1} S_{0}\right)}=-\sqrt{3} f_{S}{ }^{1} S_{0} \\
& \mathcal{P}_{22}^{\left(2^{1} S_{0} \rightarrow{ }^{3} P_{2}+{ }^{1} S_{0}\right)}=-\sqrt{3} f_{D} \quad{ }^{5} D_{0} \\
& \mathcal{P}_{L S}^{\left(2^{1} S_{0} \rightarrow^{1} P_{1}+{ }^{3} S_{1}\right)}= \begin{cases}-\sqrt{3} f_{S} & { }^{1} S_{0} \\
-\sqrt{3} f_{D} & { }^{5} D_{0}\end{cases} \\
& \mathcal{P}_{L S}^{\left(2^{1} S_{0} \rightarrow{ }^{3} P_{1}+{ }^{3} S_{1}\right)}=\left\{\begin{array}{cc}
\sqrt{6} f_{S} & { }^{1} S_{0} \\
-\sqrt{\frac{3}{2}} f_{D} & { }^{5} D_{0}
\end{array}\right. \\
& \mathcal{P}_{22}^{\left(2^{1} S_{0} \rightarrow{ }^{3} P_{2}+{ }^{3} S_{1}\right)}=\sqrt{\frac{9}{2}} f_{D} \quad{ }^{5} D_{0}
\end{aligned}
$$




$$
3 S \rightarrow 1 S+1 S
$$

(See $1 S \rightarrow 1 S+1 S$ for channel coefficients.)

$$
f_{P}=-\frac{2^{7 / 2} 5^{1 / 2} 7}{3^{11 / 2}} x\left(1-\frac{4}{15} x^{2}+\frac{4}{315} x^{4}\right)
$$

$3 S \rightarrow 2 S+1 S$

$$
f_{P}=-\frac{2^{4} 5^{3 / 2}}{3^{5}} x\left(1-\frac{1}{4} x^{2}+\frac{1}{75} x^{4}-\frac{1}{6075} x^{6}\right)
$$

$3^{3} \mathbf{S}_{1}$

$$
\begin{aligned}
\mathcal{P}_{10}^{\left(3^{3} S_{1} \rightarrow 2^{1} S_{0}+{ }^{1} S_{0}\right)} & =f_{P}{ }^{1} P_{1} \\
\mathcal{P}_{11}^{\left(3^{3} S_{1} \rightarrow 2^{3} S_{1}+{ }^{1} S_{0}\right)} & =-\sqrt{2} f_{P}{ }^{3} P_{1} \\
\mathcal{P}_{11}^{\left(3^{3} S_{1} \rightarrow 2^{1} S_{0}+{ }^{3} S_{1}\right)} & =\sqrt{2} f_{P}{ }^{3} P_{1} \\
\mathcal{P}_{L S}^{\left(3^{3} S_{1} \rightarrow 2^{3} S_{1}+{ }^{3} S_{1}\right)} & =\left\{\begin{array}{cc}
\sqrt{\frac{1}{3}} f_{P} & { }^{1} P_{1} \\
0 & { }^{3} P_{1} \\
-\sqrt{\frac{20}{3}} f_{P} & { }^{5} P_{1} \\
0 & { }^{5} F_{1}
\end{array}\right.
\end{aligned}
$$

$3^{1} S_{0}$

$$
\begin{aligned}
& \mathcal{P}_{11}^{\left(3^{1} S_{0} \rightarrow 2^{3} S_{1}+{ }^{1} S_{0}\right)}=-\sqrt{3} f_{P}{ }^{3} P_{0} \\
& \mathcal{P}_{11}^{\left(3^{1} S_{0} \rightarrow 2^{1} S_{0}+{ }^{3} S_{1}\right)}=-\sqrt{3} f_{P}{ }^{3} P_{0} \\
& \mathcal{P}_{11}^{\left(3^{1} S_{0} \rightarrow 2^{3} S_{1}+{ }^{3} S_{1}\right)}=\sqrt{6} f_{P}{ }^{3} P_{0}
\end{aligned}
$$


$3 S \rightarrow 1 P+1 S$

(See $2 S \rightarrow 1 P+1 S$ for channel coefficients.)

$$
\begin{aligned}
& f_{S}=\frac{2^{3} 5^{3 / 2}}{3^{5}}\left(1-\frac{3}{5} x^{2}+\frac{16}{225} x^{4}-\frac{4}{2025} x^{6}\right) \\
& f_{D}=\frac{2^{7 / 2} 7^{2}}{3^{6} 5^{1 / 2}} x^{2}\left(1-\frac{20}{147} x^{2}+\frac{4}{1323} x^{4}\right)
\end{aligned}
$$

$3 S \rightarrow 2 P+1 S$

(See $2 S \rightarrow 1 P+1 S$ for channel coefficients.)

$$
\begin{aligned}
& f_{S}=\frac{2^{5 / 2}}{3^{4}}\left(1-\frac{47}{18} x^{2}+\frac{1}{2} x^{4}-\frac{8}{405} x^{6}+\frac{2}{10935} x^{8}\right) \\
& f_{D}=\frac{2^{6} 5}{3^{6}} x^{2}\left(1-\frac{57}{400} x^{2}+\frac{13}{2700} x^{4}-\frac{1}{24300} x^{6}\right)
\end{aligned}
$$

$3 S \rightarrow 1 D+1^{1} S_{0}$

$$
\begin{aligned}
& f_{P}=-\frac{2^{3}}{3^{5}} x\left(1-\frac{23}{15} x^{2}+\frac{8}{45} x^{4}-\frac{4}{1215} x^{6}\right) \\
& f_{F}=-\frac{2^{5 / 2}(43)}{3^{11 / 2} 5} x^{3}\left(1-\frac{92}{1161} x^{2}+\frac{4}{3483} x^{4}\right)
\end{aligned}
$$

$3^{3} \mathbf{S}_{1}$

$$
\begin{aligned}
& \mathcal{P}_{L S}^{\left(3^{3} S_{1} \rightarrow^{1} D_{2}+{ }^{1} S_{0}\right)}= \begin{cases}f_{P} & { }^{5} P_{1} \\
f_{F} & { }^{5} F_{1}\end{cases} \\
& \mathcal{P}_{11}^{\left(3^{3} S_{1} \rightarrow^{3} D_{1}+{ }^{1} S_{0}\right)}=\sqrt{\frac{1}{2}} f_{P}{ }^{3} P_{1} \\
& \mathcal{P}_{L S}^{\left(3^{3} S_{1} \rightarrow{ }^{3} D_{2}+{ }^{1} S_{0}\right)}=\left\{\begin{array}{cc}
-\sqrt{\frac{3}{2}} f_{P} & { }^{5} P_{1} \\
\sqrt{\frac{2}{3}} f_{F} & { }^{5} F_{1}
\end{array}\right. \\
& \mathcal{P}_{33}^{\left(3^{3} S_{1} \rightarrow{ }^{3} D_{3}+{ }^{1} S_{0}\right)}=-\sqrt{\frac{4}{3}} f_{F}{ }^{7} F_{1}
\end{aligned}
$$




$$
3^{1} S_{0}
$$

$$
\begin{aligned}
& \mathcal{P}_{11}^{\left(3^{1} S_{0} \rightarrow{ }^{3} D_{1}+{ }^{1} S_{0}\right)}=-\sqrt{3} f_{P}{ }^{3} P_{0} \\
& \mathcal{P}_{33}^{\left(3^{1} S_{0} \rightarrow{ }^{3} D_{3}+{ }^{1} S_{0}\right)}=-\sqrt{3} f_{F}{ }^{7} F_{0}
\end{aligned}
$$




$$
1 P \rightarrow 1 S+1 S
$$

$$
\begin{aligned}
& f_{S}=\frac{2^{5}}{3^{5 / 2}}\left(1-\frac{2}{9} x^{2}\right) \\
& f_{D}=\frac{2^{6}}{3^{4} 5^{1 / 2}} x^{2}
\end{aligned}
$$

${ }^{3} \mathbf{P}_{2}$

$$
\begin{aligned}
\mathcal{P}_{20}^{\left(3 P_{2} \rightarrow{ }^{1} S_{0}+{ }^{1} S_{0}\right)} & =f_{D} \\
\mathcal{P}_{21}^{\left({ }^{3} P_{2} \rightarrow{ }^{3} S_{1}+{ }^{1} S_{0}\right)} & =-\sqrt{\frac{3}{2}} f_{D} \\
\mathcal{P}_{L S}^{\left({ }^{3} P_{2} \rightarrow{ }^{3} S_{1}+{ }^{3} S_{1}\right)} & = \begin{cases}-\sqrt{2} f_{S} & { }^{5} S_{2} \\
\sqrt{\frac{1}{3}} f_{D} & { }^{1} D_{2} \\
-\sqrt{\frac{7}{3}} f_{D} & { }^{5} D_{2}\end{cases}
\end{aligned}
$$

${ }^{3} \mathbf{P}_{1}$

$$
\begin{aligned}
& \mathcal{P}_{L S}^{\left({ }^{3} P_{1} \rightarrow{ }^{3} S_{1}+{ }^{1} S_{0}\right)}=\left\{\begin{array}{cc}
f_{S} & { }^{3} S_{1} \\
-\sqrt{\frac{5}{6}} f_{D} & { }^{3} D_{1}
\end{array}\right. \\
& \mathcal{P}_{L S}^{\left({ }^{3} P_{1} \rightarrow{ }^{3} S_{1}+{ }^{3} S_{1}\right)}=\left\{\begin{array}{cc}
0 & { }^{3} S_{1} \\
0 & { }^{3} D_{1} \\
-\sqrt{5} f_{D} & { }^{5} D_{1}
\end{array}\right.
\end{aligned}
$$

${ }^{3} \mathbf{P}_{0}$

$$
\mathcal{P}_{00}^{\left({ }^{3} P_{0} \rightarrow{ }^{1} S_{0}+{ }^{1} S_{0}\right)}=\sqrt{\frac{3}{2}} f_{S}{ }^{1} S_{0}
$$




$$
\begin{aligned}
\mathcal{P}_{L S}^{\left(3 P_{0} \rightarrow{ }^{3} S_{1}+{ }^{3} S_{1}\right)} & =\left\{\begin{array}{cc}
\sqrt{\frac{1}{2}} f_{S} & { }^{1} S_{0} \\
-\sqrt{\frac{20}{3}} f_{D} & { }^{5} D_{0}
\end{array}\right. \\
\mathcal{P}_{L S}^{\left({ }^{1} P_{1} \rightarrow{ }^{3} S_{1}+{ }^{1} S_{0}\right)} & =\left\{\begin{array}{cc}
-\sqrt{\frac{1}{2}} f_{S} & { }^{3} S_{1} \\
-\sqrt{\frac{5}{3}} f_{D} & { }^{3} D_{1}
\end{array}\right. \\
\mathcal{P}_{L S}^{\left({ }^{1} P_{1} \rightarrow{ }^{3} S_{1}+{ }^{3} S_{1}\right)} & =\left\{\begin{array}{cc}
f_{S} & { }^{3} S_{1} \\
\sqrt{\frac{10}{3}} f_{D} & { }^{3} D_{1} \\
0 & { }^{5} D_{1}
\end{array}\right.
\end{aligned}
$$

48 
$2 P \rightarrow 1 S+1 S$

(See $1 P \rightarrow 1 S+1 S$ for channel coefficients.)

$$
\begin{aligned}
& f_{S}=\frac{2^{9 / 2} 5^{1 / 2}}{3^{7 / 2}}\left(1-\frac{4}{9} x^{2}+\frac{4}{135} x^{4}\right) \\
& f_{D}=\frac{2^{11 / 2} 7}{3^{5} 5} x^{2}\left(1-\frac{2}{21} x^{2}\right)
\end{aligned}
$$

$2 P \rightarrow 2 S+1 S$

$$
\begin{aligned}
& f_{S}=\frac{2^{4} 5^{1 / 2} 7}{3^{5}}\left(1-\frac{1}{2} x^{2}+\frac{2}{45} x^{4}-\frac{2}{2835} x^{6}\right) \\
& f_{D}=\frac{2^{6}(11)}{3^{11 / 2} 5} x^{2}\left(1-\frac{13}{132} x^{2}+\frac{1}{594} x^{4}\right)
\end{aligned}
$$

$2^{3} \mathbf{P}_{2}$

$$
\begin{aligned}
& \mathcal{P}_{20}^{\left(2^{3} P_{2} \rightarrow 2^{1} S_{0}+{ }^{1} S_{0}\right)}=f_{D}{ }^{1} D_{2} \\
& \mathcal{P}_{21}^{\left(2^{3} P_{2} \rightarrow 2^{3} S_{1}+{ }^{1} S_{0}\right)}=-\sqrt{\frac{3}{2}} f_{D}{ }^{3} D_{2} \\
& \mathcal{P}_{21}^{\left(2^{3} P_{2} \rightarrow 2^{1} S_{0}+{ }^{3} S_{1}\right)}=+\sqrt{\frac{3}{2}} f_{D}{ }^{3} D_{2} \\
& \mathcal{P}_{L S}^{\left(2^{3} P_{2} \rightarrow 2^{3} S_{1}+{ }^{3} S_{1}\right)}=\left\{\begin{array}{cc}
-\sqrt{2} f_{S} & { }^{5} S_{2} \\
\sqrt{\frac{1}{3}} f_{D} & { }^{1} D_{2} \\
0 & { }^{3} D_{2} \\
-\sqrt{\frac{7}{3}} f_{D} & { }^{5} D_{2} \\
0 & { }^{5} G_{2}
\end{array}\right.
\end{aligned}
$$

$2^{3} \mathbf{P}_{1}$ 


$$
\begin{aligned}
& \mathcal{P}_{L S}^{\left(2^{3} P_{1} \rightarrow 2^{3} S_{1}+{ }^{1} S_{0}\right)}=\left\{\begin{array}{cc}
f_{S} & { }^{3} S_{1} \\
-\sqrt{\frac{5}{6}} f_{D} & { }^{3} D_{1}
\end{array}\right. \\
& \mathcal{P}_{L S}^{\left(2^{3} P_{1} \rightarrow 2^{1} S_{0}+{ }^{3} S_{1}\right)}=\left\{\begin{array}{cc}
-f_{S} & { }^{3} S_{1} \\
\sqrt{\frac{5}{6}} f_{D} & { }^{3} D_{1}
\end{array}\right. \\
& \mathcal{P}_{22}^{\left(2^{3} P_{1} \rightarrow 2^{3} S_{1}+{ }^{3} S_{1}\right)}=-\sqrt{5} f_{D} \quad{ }^{5} D_{1}
\end{aligned}
$$

$2^{3} \mathbf{P}_{0}$

$$
\begin{aligned}
\mathcal{P}_{00}^{\left(2^{3} P_{0} \rightarrow 2^{1} S_{0}+{ }^{1} S_{0}\right)} & =\sqrt{\frac{3}{2}} f_{S}{ }^{1} S_{0} \\
\mathcal{P}_{L S}^{\left(2^{3} P_{0} \rightarrow 2^{3} S_{1}+{ }^{3} S_{1}\right)} & =\left\{\begin{array}{cc}
\sqrt{\frac{1}{2}} f_{S} & { }^{1} S_{0} \\
-\sqrt{\frac{20}{3}} f_{D} & { }^{5} D_{0}
\end{array}\right.
\end{aligned}
$$

$2^{1} \mathbf{P}_{1}$

$$
\begin{aligned}
& \mathcal{P}_{L S}^{\left(2^{1} P_{1} \rightarrow 2^{3} S_{1}+{ }^{1} S_{0}\right)}= \begin{cases}-\sqrt{\frac{1}{2}} f_{S} & { }^{3} S_{1} \\
-\sqrt{\frac{5}{3}} f_{D} & { }^{3} D_{1}\end{cases} \\
& \mathcal{P}_{L S}^{\left(2^{1} P_{1} \rightarrow 2^{1} S_{0}+{ }^{3} S_{1}\right)}=\left\{\begin{array}{cc}
-\sqrt{\frac{1}{2}} f_{S} & { }^{3} S_{1} \\
-\sqrt{\frac{5}{3}} f_{D} & { }^{3} D_{1}
\end{array}\right. \\
& \mathcal{P}_{L S}^{\left(2^{1} P_{1 \rightarrow 2}{ }^{3} S_{1}+{ }^{3} S_{1}\right)}=\left\{\begin{array}{cc}
f_{S} & { }^{3} S_{1} \\
\sqrt{\frac{10}{3}} f_{D} & { }^{3} D_{1} \\
0 & { }^{5} D_{1}
\end{array}\right.
\end{aligned}
$$

$$
2 P \rightarrow 1 P+1 S
$$

$2^{3} P_{2}$ 


$$
\begin{aligned}
& \mathcal{P}_{L S}^{\left(2^{3} P_{2} \rightarrow^{1} P_{1}+{ }^{1} S_{0}\right)}=\left\{\begin{array}{cc}
-\frac{2^{9 / 2}(13)}{3^{5} 5^{1 / 2}} x\left(1-\frac{8}{39} x^{2}+\frac{4}{585} x^{4}\right) & { }^{3} P_{2} \\
-\frac{2^{5}}{3^{9 / 2} 5^{1 / 2}} x^{3}\left(1-\frac{2}{45} x^{2}\right) & { }^{3} F_{2}
\end{array}\right. \\
& \mathcal{P}_{L S}^{\left(2^{3} P_{2} \rightarrow{ }^{3} P_{1}+{ }^{1} S_{0}\right)}= \begin{cases}\frac{2^{6}}{3^{4} 5^{1 / 2}} x\left(1-\frac{1}{4} x^{2}+\frac{1}{90} x^{4}\right) & { }^{3} P_{2} \\
-\frac{2^{9 / 2}}{3^{9 / 2} 5^{1 / 2}} x^{3}\left(1-\frac{2}{45} x^{2}\right) & { }^{3} F_{2}\end{cases} \\
& \mathcal{P}_{L S}^{\left(2^{3} P_{2} \rightarrow^{3} P_{2}+{ }^{1} S_{0}\right)}=\left\{\begin{array}{cc}
\frac{2^{5} 7}{3^{9 / 2} 5^{1 / 2}} x\left(1-\frac{1}{6} x^{2}+\frac{1}{315} x^{4}\right) & { }^{5} P_{2} \\
\frac{2^{5}}{3^{9 / 2} 5^{1 / 2}} x^{3}\left(1-\frac{2}{45} x^{2}\right) & { }^{5} F_{2}
\end{array}\right. \\
& \mathcal{P}_{L S}^{\left(2^{3} P_{2} \rightarrow{ }^{1} P_{1}+{ }^{3} S_{1}\right)}=\left\{\begin{array}{cc}
-\frac{2^{6}}{3^{4} 5^{1 / 2}} x\left(1-\frac{1}{4} x^{2}+\frac{1}{80} x^{4}\right) & { }^{3} P_{2} \\
-\frac{2^{5} 7}{3^{9 / 2} 5^{1 / 2}} x\left(1-\frac{1}{6} x^{2}+\frac{1}{315} x^{4}\right) & { }^{5} P_{2} \\
\frac{2^{9 / 2}}{3^{9 / 2} 5^{1 / 2}} x^{3}\left(1-\frac{2}{45} x^{2}\right) & { }^{3} F_{2} \\
-\frac{2^{5}}{3^{9 / 2} 5^{1 / 2}} x^{3}\left(1-\frac{2}{45} x^{2}\right) & { }^{5} F_{2}
\end{array}\right. \\
& \mathcal{P}_{L S}^{\left(2^{3} P_{2} \rightarrow{ }^{3} P_{0}+{ }^{3} S_{1}\right)}=\left\{\begin{array}{cc}
\frac{2^{11 / 2} 5^{1 / 2}}{3^{11 / 2}} x\left(1-\frac{11}{30} x^{2}+\frac{1}{45} x^{4}\right) & { }^{3} P_{2} \\
0 & { }^{3} F_{2}
\end{array}\right. \\
& \mathcal{P}_{L S}^{\left(2^{3} P_{2} \rightarrow{ }^{3} P_{1}+{ }^{3} S_{1}\right)}=\left\{\begin{array}{cc}
\frac{2^{7 / 2}(23)}{3^{5} 5^{1 / 2}} x\left(1-\frac{19}{69} x^{2}+\frac{14}{1035} x^{4}\right) & { }^{3} P_{2} \\
\frac{2^{7 / 2}}{3^{9 / 2} 5^{1 / 2}} x\left(1+\frac{1}{3} x^{2}-\frac{2}{45} x^{4}\right) & { }^{5} P_{2} \\
\frac{2^{4}}{3^{9 / 2} 5^{1 / 2}} x^{3}\left(1-\frac{2}{45} x^{2}\right) & { }^{3} F_{2} \\
-\frac{2^{9 / 2}}{3^{9 / 2} 5^{1 / 2}} x^{3}\left(1-\frac{2}{45} x^{2}\right) & { }^{5} F_{2}
\end{array}\right. \\
& \mathcal{P}_{L S}^{\left(2^{3} P_{2} \rightarrow{ }^{3} P_{2}+{ }^{3} S_{1}\right)}=\left\{\begin{array}{cc}
-\frac{2^{7 / 2}(29)}{3^{11 / 25}} x\left(1-\frac{13}{87} x^{2}+\frac{2}{1305} x^{4}\right) & { }^{3} P_{2} \\
\frac{2^{7 / 2}}{3^{5} 5^{1 / 2}} x\left(1+\frac{1}{3} x^{2}-\frac{2}{45} x^{4}\right) & { }^{5} P_{2} \\
\frac{2^{9 / 2} 7^{1 / 2}(41)}{3^{55}} x\left(1-\frac{22}{123} x^{2}+\frac{8}{1845} x^{4}\right) & { }^{7} P_{2} \\
-\frac{2^{4}}{3^{4} 5} x^{3}\left(1-\frac{2}{45} x^{2}\right) & { }^{3} F_{2} \\
-\frac{2^{9 / 2}}{3^{5} 5^{1 / 2}} x^{3}\left(1-\frac{2}{45} x^{2}\right) & { }^{5} F_{2} \\
\frac{2^{13 / 2}}{3^{9 / 2}} x^{3}\left(1-\frac{2}{45} x^{2}\right) & { }^{7} F_{2} \\
0 & { }^{7} H_{2}
\end{array}\right.
\end{aligned}
$$




$$
\begin{aligned}
& \mathcal{P}_{11}^{\left(2^{3} P_{1} \rightarrow{ }^{1} P_{1}+{ }^{1} S_{0}\right)}=\frac{2^{9 / 2} 5^{1 / 2}}{3^{4}} x\left(1-\frac{2}{15} x^{2}\right) \quad{ }^{3} P_{1} \\
& \mathcal{P}_{10}^{\left(2^{3} P_{1} \rightarrow{ }^{3} P_{0}+{ }^{1} S_{0}\right)}=\frac{2^{4} 5^{1 / 2}}{3^{9 / 2}} x\left(1-\frac{2}{15} x^{2}\right) \quad{ }^{1} P_{1} \\
& \mathcal{P}_{11}^{\left(2^{3} P_{1} \rightarrow^{3} P_{1}+{ }^{1} S_{0}\right)}=\frac{2^{4} 5^{3 / 2}}{3^{5}} x\left(1-\frac{17}{75} x^{2}+\frac{2}{225} x^{4}\right) \quad{ }^{3} P_{1} \\
& \mathcal{P}_{L S}^{\left(2^{3} P_{1} \rightarrow{ }^{3} P_{2}+{ }^{1} S_{0}\right)}=\left\{\begin{array}{cc}
-\frac{2^{4}}{3^{5 / 2}} x\left(1-\frac{5}{27} x^{2}+\frac{2}{405} x^{4}\right) & { }^{5} P_{1} \\
\frac{2^{9 / 2}}{3^{5}} x^{3}\left(1-\frac{2}{45} x^{2}\right) & { }^{5} F_{1}
\end{array}\right. \\
& \mathcal{P}_{L S}^{\left(2^{3} P_{1} \rightarrow^{1} P_{1}+^{3} S_{1}\right)}=\left\{\begin{array}{cc}
-\frac{2^{4} 5^{1 / 2}}{3^{9 / 2}} x\left(1-\frac{2}{15} x^{2}\right) & { }^{1} P_{1} \\
-\frac{2^{4} 5^{3 / 2}}{3^{5}} x\left(1-\frac{17}{75} x^{2}+\frac{2}{225} x^{4}\right) & { }^{3} P_{1} \\
\frac{2^{4}}{3^{5 / 2}} x\left(1-\frac{5}{27} x^{2}+\frac{2}{405} x^{4}\right) & { }^{5} P_{1} \\
-\frac{2^{9 / 2}}{3^{5}} x^{3}\left(1-\frac{2}{45} x^{2}\right) & { }^{5} F_{1}
\end{array}\right. \\
& \mathcal{P}_{11}^{\left(2^{3} P_{1} \rightarrow{ }^{3} P_{0}+{ }^{3} S_{1}\right)}=\frac{2^{9 / 2} 5^{3 / 2}}{3^{11 / 2}} x\left(1-\frac{17}{75} x^{2}+\frac{2}{225} x^{4}\right) \quad{ }^{3} P_{1} \\
& \mathcal{P}_{L S}^{\left(2^{3} P_{1} \rightarrow^{3} P_{1}+^{3} S_{1}\right)}=\left\{\begin{array}{cc}
0 & { }^{1} P_{1} \\
\frac{2^{9 / 2} 5^{1 / 2}}{3^{5}} x\left(1-\frac{11}{30} x^{2}+\frac{1}{45} x^{4}\right) & { }^{3} P_{1} \\
\frac{2^{9 / 2} 7}{3^{9 / 2}} x\left(1-\frac{1}{6} x^{2}+\frac{1}{315} x^{4}\right) & { }^{5} P_{1} \\
-\frac{2^{4}}{3^{5}} x^{3}\left(1-\frac{2}{45} x^{2}\right) & { }^{5} F_{1}
\end{array}\right. \\
& \mathcal{P}_{L S}^{\left(2^{3} P_{1} \rightarrow{ }^{3} P_{2}+{ }^{3} S_{1}\right)}=\left\{\begin{array}{cc}
\frac{2^{9 / 2} 7}{3^{11 / 2}} x\left(1-\frac{1}{6} x^{2}+\frac{1}{315} x^{4}\right) & { }^{3} P_{1} \\
\frac{2^{9 / 2} 7}{3^{5}} x\left(1-\frac{1}{6} x^{2}+\frac{1}{315} x^{4}\right) & { }^{5} P_{1} \\
-\frac{2^{4}}{3^{11 / 2}} x^{3}\left(1-\frac{2}{45} x^{2}\right) & { }^{5} F_{1} \\
\frac{2^{13 / 2}}{3^{11 / 2}} x^{3}\left(1-\frac{2}{45} x^{2}\right) & { }^{7} F_{1}
\end{array}\right.
\end{aligned}
$$

$2^{3} \mathbf{P}_{0}$

$$
\begin{aligned}
& \mathcal{P}_{11}^{\left(2^{3} P_{0} \rightarrow{ }^{1} P_{1}+{ }^{1} S_{0}\right)}=-\frac{2^{7 / 2} 5^{1 / 2}(13)}{3^{5}} x\left(1-\frac{8}{39} x^{2}+\frac{4}{585} x^{4}\right){ }^{3} P_{0} \\
& \mathcal{P}_{11}^{\left(2^{3} P_{0} \rightarrow{ }^{3} P_{1}+{ }^{1} S_{0}\right)}=-\frac{2^{5} 5^{1 / 2}}{3^{4}} x\left(1-\frac{2}{15} x^{2}\right){ }^{3} P_{0} \\
& \mathcal{P}_{11}^{\left(2^{3} P_{0} \rightarrow{ }^{1} P_{1}+{ }^{3} S_{1}\right)}=\frac{2^{5} 5^{1 / 2}}{3^{4}} x\left(1-\frac{2}{15} x^{2}\right){ }^{3} P_{0} \\
& \mathcal{P}_{11}^{\left(2^{3} P_{0} \rightarrow{ }^{3} P_{0}+{ }^{3} S_{1}\right)}=\frac{2^{7 / 2} 5^{1 / 2}(13)}{3^{11 / 2}} x\left(1-\frac{8}{39} x^{2}+\frac{4}{585} x^{4}\right) \quad{ }^{3} P_{0}
\end{aligned}
$$




$$
\begin{aligned}
& \mathcal{P}_{11}^{\left(2^{3} P_{0} \rightarrow{ }^{3} P_{1}+{ }^{3} S_{1}\right)}=\frac{2^{7 / 2} 5^{1 / 2}(13)}{3^{5}} x\left(1-\frac{8}{39} x^{2}+\frac{4}{585} x^{4}\right) \quad{ }^{3} P_{0} \\
& \mathcal{P}_{L S}^{\left(2^{3} P_{0} \rightarrow{ }^{3} P_{2}+{ }^{3} S_{1}\right)}=\left\{\begin{array}{cc}
-\frac{2^{7 / 2}(13)}{3^{11 / 2} x\left(1-\frac{8}{39} x^{2}+\frac{4}{585} x^{4}\right)} & { }^{3} P_{0} \\
\frac{2^{6}}{3^{5}} x^{3}\left(1-\frac{2}{45} x^{2}\right) & { }^{7} F_{0}
\end{array}\right.
\end{aligned}
$$

$2^{1} \mathbf{P}_{1}$

$$
\begin{aligned}
& \mathcal{P}_{11}^{\left(2^{1} P_{1} \rightarrow^{1} P_{1}+{ }^{1} S_{0}\right)}=0 \quad{ }^{3} P_{1} \\
& \mathcal{P}_{10}^{\left(2^{1} P_{1} \rightarrow{ }^{3} P_{0}+{ }^{1} S_{0}\right)}=\frac{2^{7 / 2} 5^{1 / 2} 7}{3^{11 / 2}} x\left(1-\frac{4}{15} x^{2}+\frac{4}{315} x^{4}\right) \quad{ }^{1} P_{1} \\
& \mathcal{P}_{10}^{\left(2^{1} P_{1} \rightarrow{ }^{3} P_{1}+{ }^{1} S_{0}\right)}=\frac{2^{7 / 2} 5^{1 / 2}}{3^{4}} x\left(1-\frac{2}{15} x^{2}\right){ }^{1} P_{1} \\
& \mathcal{P}_{L S}^{\left(2^{1} P_{1} \rightarrow{ }^{3} P_{2}+{ }^{1} S_{0}\right)}=\left\{\begin{array}{cc}
\frac{2^{7 / 2}(41)}{3^{11 / 2}} x\left(1-\frac{22}{123} x^{2}+\frac{8}{1845} x^{4}\right) & { }^{5} P_{1} \\
\frac{2^{5}}{3^{5}} x^{3}\left(1-\frac{2}{45} x^{2}\right) & { }^{5} F_{1}
\end{array}\right. \\
& \mathcal{P}_{L S}^{\left(2^{1} P_{1} \rightarrow^{1} P_{1}+{ }^{3} S_{1}\right)}=\left\{\begin{array}{cc}
\frac{2^{7 / 2} 5^{1 / 2} 7}{3^{11 / 2}} x\left(1-\frac{4}{15} x^{2}+\frac{4}{315} x^{4}\right) & { }^{1} P_{1} \\
\frac{2^{7 / 2} 5^{1 / 2}}{3^{4}} x\left(1-\frac{2}{15} x^{2}\right) & { }^{3} P_{1} \\
\frac{2^{7 / 2}(41)}{3^{11 / 2}} x\left(1-\frac{22}{123} x^{2}+\frac{8}{1845} x^{4}\right) & { }^{5} P_{1} \\
\frac{2^{5}}{3^{5}} x^{3}\left(1-\frac{2}{45} x^{2}\right) & { }^{5} F_{1}
\end{array}\right. \\
& \mathcal{P}_{11}^{\left(2^{1} P_{1} \rightarrow^{3} P_{0}+^{3} S_{1}\right)}=\frac{2^{4} 5^{1 / 2}}{3^{9 / 2}} x\left(1-\frac{2}{15} x^{2}\right) \quad{ }^{3} P_{1}
\end{aligned}
$$




$$
1 D \rightarrow 1 S+1 S
$$

$$
\begin{aligned}
& f_{P}=\frac{2^{13 / 2}}{3^{4}} x\left(1-\frac{2}{15} x^{2}\right) \\
& f_{F}=-\frac{2^{6}}{3^{9 / 2} 5^{1 / 2} 7^{1 / 2}} x^{3}
\end{aligned}
$$

\section{${ }^{3} \mathrm{D}_{3}$}

$$
\begin{aligned}
& \mathcal{P}_{30}^{\left({ }^{3} D_{3} \rightarrow{ }^{1} S_{0}+{ }^{1} S_{0}\right)}=f_{F} \quad{ }^{1} F_{3} \\
& \mathcal{P}_{31}^{\left({ }^{3} D_{3} \rightarrow{ }^{3} S_{1}+{ }^{1} S_{0}\right)}=-\sqrt{\frac{4}{3}} f_{F} \quad{ }^{3} F_{3} \\
& \mathcal{P}_{L S}^{\left({ }^{3} D_{3} \rightarrow{ }^{3} S_{1}+{ }^{3} S_{1}\right)}=\left\{\begin{array}{cc}
f_{P} & { }^{5} P_{3} \\
\sqrt{\frac{1}{3}} f_{F} & { }^{1} F_{3} \\
0 & { }^{3} F_{3} \\
-\sqrt{\frac{8}{5}} f_{F} & { }^{5} F_{3} \\
0 & { }^{5} H_{3}
\end{array}\right.
\end{aligned}
$$

${ }^{3} \mathrm{D}_{2}$

$$
\begin{aligned}
\mathcal{P}_{L S}^{\left({ }^{3} D_{2} \rightarrow{ }^{3} S_{1}+{ }^{1} S_{0}\right)} & =\left\{\begin{array}{cc}
-\sqrt{\frac{3}{8}} f_{P} & { }^{3} P_{2} \\
-\sqrt{\frac{14}{15}} f_{F} & { }^{3} F_{2}
\end{array}\right. \\
\mathcal{P}_{L S}^{\left({ }^{3} D_{2} \rightarrow{ }^{3} S_{1}+{ }^{3} S_{1}\right)} & =\left\{\begin{array}{cc}
\frac{1}{2} f_{P} & { }^{5} P_{2} \\
0 & { }^{3} F_{2} \\
-\sqrt{\frac{56}{15}} f_{F} & { }^{5} F_{2}
\end{array}\right.
\end{aligned}
$$

${ }^{3} \mathrm{D}_{1}$ 


$$
\begin{aligned}
& \mathcal{P}_{10}^{\left({ }^{3} D_{1} \rightarrow^{1} S_{0}+{ }^{1} S_{0}\right)}=-\sqrt{\frac{5}{12}} f_{P} \quad{ }^{1} P_{1} \\
& \mathcal{P}_{11}^{\left({ }^{3} D_{1} \rightarrow{ }^{3} S_{1}+{ }^{1} S_{0}\right)}=-\sqrt{\frac{5}{24}} f_{P}{ }^{3} P_{1} \\
& \mathcal{P}_{L S}^{\left({ }^{3} D_{1} \rightarrow{ }^{3} S_{1}+{ }^{3} S_{1}\right)}=\left\{\begin{array}{cc}
-\frac{\sqrt{5}}{6} f_{P} & { }^{1} P_{1} \\
0 & { }^{3} P_{1} \\
\frac{1}{6} f_{P} & { }^{5} P_{1} \\
-\sqrt{\frac{28}{5}} f_{F} & { }^{5} F_{1}
\end{array}\right.
\end{aligned}
$$

${ }^{1} \mathrm{D}_{2}$

$$
\begin{aligned}
& \mathcal{P}_{L S}^{\left({ }^{1} D_{2} \rightarrow{ }^{3} S_{1}+{ }^{1} S_{0}\right)}=\left\{\begin{array}{cc}
\frac{1}{2} f_{P} & { }^{3} P_{2} \\
-\sqrt{\frac{7}{5}} f_{F} & { }^{3} F_{2}
\end{array}\right. \\
& \mathcal{P}_{L S}^{\left({ }^{1} D_{2} \rightarrow{ }^{3} S_{1}+{ }^{3} S_{1}\right)}=\left\{\begin{array}{cc}
-\sqrt{\frac{1}{2}} f_{P} & { }^{3} P_{2} \\
0 & { }^{5} P_{2} \\
\sqrt{\frac{14}{5}} f_{F} & { }^{3} F_{2} \\
0 & { }^{5} F_{2}
\end{array}\right.
\end{aligned}
$$

$$
1 D \rightarrow 2 S+1 S
$$

$$
\begin{aligned}
& f_{P}=\frac{2^{6}}{3^{11 / 2}} x\left(1-\frac{29}{30} x^{2}+\frac{1}{45} x^{4}\right) \\
& f_{F}=-\frac{2^{13 / 2}}{3^{5} 5^{1 / 2} 7^{1 / 2}} x^{3}\left(1-\frac{1}{36} x^{2}\right)
\end{aligned}
$$

${ }^{3} \mathrm{D}_{3}$

$$
\begin{aligned}
& \mathcal{P}_{30}^{\left({ }^{3} D_{3 \rightarrow 2} 2^{1} S_{0}+{ }^{1} S_{0}\right)}=f_{F} \quad{ }^{1} F_{3} \\
& \mathcal{P}_{31}^{\left({ }^{3} D_{3} \rightarrow 2^{3} S_{1}+{ }^{1} S_{0}\right)}=-\sqrt{\frac{4}{3}} f_{F}
\end{aligned}
$$




$$
\begin{aligned}
\mathcal{P}_{31}^{\left({ }^{3} D_{3} \rightarrow 2^{1} S_{0}+{ }^{3} S_{1}\right)} & =\sqrt{\frac{4}{3}} f_{F} \\
\mathcal{P}_{L S}^{\left({ }^{3} D_{3} \rightarrow 2^{3} S_{1}+{ }^{3} S_{1}\right)} & =\left\{\begin{array}{cc}
f_{P} & { }^{5} P_{3} \\
\sqrt{\frac{1}{3}} f_{F} & { }^{1} F_{3} \\
0 & { }^{3} F_{3} \\
-\sqrt{\frac{8}{5}} f_{F} & { }^{5} F_{3} \\
0 & { }^{5} H_{3}
\end{array}\right.
\end{aligned}
$$

${ }^{3} \mathrm{D}_{2}$

$$
\begin{aligned}
\mathcal{P}_{L S}^{\left({ }^{3} D_{2} \rightarrow 2^{3} S_{1}+{ }^{1} S_{0}\right)} & =\left\{\begin{array}{cc}
-\sqrt{\frac{3}{8}} f_{P} & { }^{3} P_{2} \\
-\sqrt{\frac{14}{15}} f_{F} & { }^{3} F_{2}
\end{array}\right. \\
\mathcal{P}_{L S}^{\left({ }^{3} D_{2} \rightarrow 2^{2} S_{0}+{ }^{3} S_{1}\right)} & =\left\{\begin{array}{cc}
\sqrt{\frac{3}{8}} f_{P} & { }^{3} P_{2} \\
\sqrt{\frac{14}{15}} f_{F} & { }^{3} F_{2}
\end{array}\right. \\
\mathcal{P}_{L S}^{\left({ }^{3} D_{2 \rightarrow 2^{3}} S_{1}+{ }^{3} S_{1}\right)} & =\left\{\begin{array}{cc}
0 & { }^{3} P_{2} \\
\frac{1}{2} f_{P} & { }^{5} P_{2} \\
0 & { }^{3} F_{2} \\
-\sqrt{\frac{56}{15}} f_{F} & { }^{5} F_{2}
\end{array}\right.
\end{aligned}
$$

${ }^{3} \mathbf{D}_{1}$

$$
\begin{aligned}
& \mathcal{P}_{10}^{\left({ }^{3} D_{1} \rightarrow 2^{1} S_{0}+{ }^{1} S_{0}\right)}=-\sqrt{\frac{5}{12}} f_{P} \quad{ }^{1} P_{1} \\
& \mathcal{P}_{11}^{\left({ }^{3} D_{1} \rightarrow 2^{3} S_{1}+{ }^{1} S_{0}\right)}=-\sqrt{\frac{5}{24}} f_{P} \quad{ }^{1} P_{1} \\
& \mathcal{P}_{11}^{\left({ }^{3} D_{1} \rightarrow 2^{1} S_{0}+{ }^{3} S_{1}\right)}=\sqrt{\frac{5}{24}} f_{P} \quad{ }^{1} P_{1} \\
& \mathcal{P}_{L S}^{\left({ }^{3} D_{1} \rightarrow 2^{3} S_{1}+{ }^{3} S_{1}\right)}=\left\{\begin{array}{cc}
-\sqrt{\frac{5}{36}} f_{P} & { }^{1} P_{1} \\
0 & { }^{3} P_{1} \\
\frac{1}{6} f_{P} & { }^{5} P_{1} \\
-\sqrt{\frac{28}{5}} f_{F} & { }^{5} F_{1}
\end{array}\right.
\end{aligned}
$$


${ }^{1} \mathrm{D}_{2}$

$$
\begin{aligned}
& \mathcal{P}_{L S}^{\left({ }^{1} D_{2 \rightarrow 2^{3}} S_{1}+{ }^{1} S_{0}\right)}=\left\{\begin{array}{cc}
\frac{1}{2} f_{P} & { }^{3} P_{2} \\
-\sqrt{\frac{7}{5}} f_{F} & { }^{3} F_{2}
\end{array}\right. \\
& \mathcal{P}_{L S}^{\left({ }^{1} D_{2} \rightarrow 2^{1} S_{0}+{ }^{3} S_{1}\right)}=\left\{\begin{array}{cc}
\frac{1}{2} f_{P} & { }^{3} P_{2} \\
-\sqrt{\frac{7}{5}} f_{F} & { }^{3} F_{2}
\end{array}\right. \\
& \mathcal{P}_{L S}^{\left({ }^{1} D_{2} \rightarrow 2^{3} S_{1}+{ }^{3} S_{1}\right)}=\left\{\begin{array}{cc}
-\sqrt{\frac{1}{2}} f_{P} & { }^{3} P_{2} \\
0 & { }^{5} P_{2} \\
\sqrt{\frac{14}{5}} f_{F} & { }^{3} F_{2} \\
0 & { }^{5} F_{2}
\end{array}\right.
\end{aligned}
$$

$$
1 D \rightarrow 1 P+1 S
$$

${ }^{3} \mathrm{D}_{3}$

$$
\begin{aligned}
& \mathcal{P}_{L S}^{\left({ }^{3} D_{3} \rightarrow{ }^{1} P_{1}+{ }^{1} S_{0}\right)}=\left\{\begin{array}{cc}
\frac{2^{11 / 2}}{3^{4} 5^{1 / 2}} x^{2}\left(1-\frac{1}{21} x^{2}\right) & { }^{3} D_{3} \\
\frac{2^{13 / 2}}{3^{11 / 2} 5^{1 / 2}} x^{4} & { }^{3} G_{3}
\end{array}\right. \\
& \mathcal{P}_{L S}^{\left({ }^{3} D_{3} \rightarrow{ }^{3} P_{1}+{ }^{1} S_{0}\right)}=\left\{\begin{array}{cc}
-\frac{2^{6}}{3^{5} 5^{1 / 2}} x^{2}\left(1-\frac{2}{21} x^{2}\right) & { }^{3} D_{3} \\
\frac{2^{6}}{3^{11 / 2} 5^{1 / 2}} x^{4} & { }^{3} G_{3}
\end{array}\right. \\
& \mathcal{P}_{L S}^{\left({ }^{3} D_{3} \rightarrow{ }^{3} P_{2}+{ }^{1} S_{0}\right)}=\left\{\begin{array}{cc}
-\frac{2^{15 / 2}}{3^{5} 5^{1 / 2}} x^{2}\left(1-\frac{1}{42} x^{2}\right) & { }^{5} D_{3} \\
-\frac{2^{6}}{3^{6} 7} x^{4} & { }^{5} G_{3}
\end{array}\right.
\end{aligned}
$$




$$
\begin{aligned}
\mathcal{P}_{L S}^{\left({ }^{3} D_{3} \rightarrow{ }^{1} P_{1}+{ }^{3} S_{1}\right)}=\left\{\begin{array}{cc}
\frac{2^{6}}{3^{55^{1 / 2}}} x^{2}\left(1-\frac{2}{21} x^{2}\right) & { }^{3} D_{3} \\
\frac{2^{15 / 2}}{3^{5} 5^{1 / 2}} x^{2}\left(1-\frac{1}{42} x^{2}\right) & { }^{5} D_{3} \\
-\frac{2^{6}}{3^{11 / 2} 5^{1 / 2} 7} x^{4} & { }^{3} G_{3} \\
\frac{2^{6}}{3^{67}} x^{4} & { }^{5} G_{3}
\end{array}\right. \\
\mathcal{P}_{21}^{\left({ }^{3} D_{3} \rightarrow{ }^{3} P_{0}+^{3} S_{1}\right)}=\left\{\begin{array}{cc}
\frac{2^{11 / 2}}{3^{11 / 2} 5^{1 / 2}} x^{2}\left(1+\frac{1}{3} x^{2}\right) & { }^{3} D_{3} \\
0 & { }^{3} G_{3}
\end{array}\right. \\
\mathcal{P}_{L S}^{\left({ }^{3} D_{3} \rightarrow{ }^{3} P_{1}+{ }^{3} S_{1}\right)}=\left\{\begin{array}{cc}
-\frac{2^{11 / 2}}{3^{5} 5^{1 / 2}} x^{2}\left(1-\frac{5}{21} x^{2}\right) & { }^{3} D_{3} \\
-\frac{2^{6}}{3^{5} 5^{1 / 2}} x^{2}\left(1+\frac{1}{21} x^{2}\right) & { }^{5} D_{3} \\
-\frac{2^{11 / 2}}{3^{11 / 2} 5^{1 / 2} 7} x^{4} & { }^{3} G_{3} \\
\frac{2^{11 / 2}}{3^{6} 7} x^{4} & { }^{5} G_{3}
\end{array}\right. \\
\mathcal{P}_{L S}^{\left({ }^{3} D_{3} \rightarrow{ }^{3} P_{2}+{ }^{3} S_{1}\right)}=\left\{\begin{array}{cc}
{ }^{7} S_{3} \\
-\frac{2^{7}}{3^{7 / 2}}\left(1-\frac{5}{18} x^{2}+\frac{1}{135} x^{4}\right) & { }^{7} S_{3} \\
\frac{2^{11 / 2}}{3^{11 / 2}} x^{2}\left(1-\frac{1}{105} x^{2}\right) & { }^{3} D_{3} \\
-\frac{2^{6}}{3^{11 / 2} 5^{1 / 2}} x^{2}\left(1+\frac{1}{21} x^{2}\right) & { }^{5} D_{3} \\
-\frac{2^{7}}{3^{5}} x^{2}\left(1-\frac{4}{105} x^{2}\right) & { }^{7} D_{3} \\
\frac{2^{11 / 2}}{3^{5} 57} x^{4} & \frac{2^{11 / 2}}{3^{13 / 2} 7} x^{4} \\
-\frac{2^{15 / 2}(11)^{1 / 2}}{3^{13 / 2} 7} x^{4} & { }^{7} G_{3}
\end{array}\right.
\end{aligned}
$$

${ }^{3} \mathrm{D}_{2}$

$$
\begin{aligned}
& \mathcal{P}_{21}^{\left({ }^{3} D_{2} \rightarrow^{1} P_{1}+{ }^{1} S_{0}\right)}=-\frac{2^{11 / 2}}{3^{4} 5^{1 / 2}} x^{2} \quad{ }^{3} D_{2} \\
& \mathcal{P}_{20}^{\left({ }^{3} D_{2} \rightarrow{ }^{3} P_{0}+{ }^{1} S_{0}\right)}=-\frac{2^{5}}{3^{4} 5^{1 / 2}} \dot{x}^{2}{ }^{1} D_{2} \\
& \mathcal{P}_{21}^{\left({ }^{3} D_{2} \rightarrow{ }^{3} P_{1}+{ }^{1} S_{0}\right)}=-\frac{2^{4} 7}{3^{5} 5^{1 / 2}} x^{2}\left(1-\frac{2}{21} x^{2}\right){ }^{3} D_{2} \\
& \mathcal{P}_{L S}^{\left({ }^{3} D_{2 \rightarrow} \rightarrow^{3} P_{2}+{ }^{1} S_{0}\right)}=\left\{\begin{array}{cc}
\frac{2^{11 / 2}}{3^{3}}\left(1-\frac{5}{18} x^{2}+\frac{1}{135} x^{4}\right) & { }^{5} S_{2} \\
\frac{2^{4} 7^{1 / 2}}{3^{5} 5^{1 / 2}} x^{2}\left(1-\frac{2}{21} x^{2}\right) & { }^{5} D_{2} \\
-\frac{2^{7}}{3^{6} 57^{1 / 2}} x^{4} & { }^{5} G_{2}
\end{array}\right.
\end{aligned}
$$




$$
\begin{aligned}
& \mathcal{P}_{L S}^{\left({ }^{3} D_{2} \rightarrow^{1} P_{1}+{ }^{3} S_{1}\right)}=\left\{\begin{array}{cc}
-\frac{2^{11 / 2}}{3^{3}}\left(1-\frac{5}{18} x^{2}+\frac{1}{135} x^{4}\right) & { }^{5} S_{2} \\
\frac{2^{5}}{3^{4} 5^{1 / 2}} x^{2} & { }^{1} D_{2} \\
\frac{2^{4} 7}{3^{5} 5^{1 / 2}} x^{2}\left(1-\frac{2}{21} x^{2}\right) & { }^{3} D_{2} \\
-\frac{2^{4} 7^{1 / 2}}{3^{5} 5^{1 / 2}} x^{2}\left(1-\frac{2}{21} x^{2}\right) & { }^{5} D_{2} \\
\frac{2^{7}}{3^{6} 7^{1 / 2}} x^{4} & { }^{5} G_{2}
\end{array}\right. \\
& \mathcal{P}_{21}^{\left({ }^{3} D_{2} \rightarrow{ }^{3} P_{0}+{ }^{3} S_{1}\right)}=-\frac{2^{9 / 2} 7}{3^{11 / 2} 5^{1 / 2}} x^{2}\left(1-\frac{2}{21} x^{2}\right){ }^{3} D_{2} \\
& \mathcal{P}_{L S}^{\left({ }^{3} D_{2} \rightarrow{ }^{3} P_{1}+{ }^{3} S_{1}\right)}=\left\{\begin{array}{cc}
-\frac{2^{5}}{3^{3}}\left(1-\frac{5}{18} x^{2}+\frac{1}{135} x^{4}\right) & { }^{5} S_{2} \\
-\frac{2^{7 / 2}}{3^{5} 5^{1 / 2}} x^{2}\left(1-\frac{2}{3} x^{2}\right) & { }^{3} D_{2} \\
-\frac{2^{7 / 2} 7^{3 / 2}}{3^{5} 5^{1 / 2}} x^{2}\left(1-\frac{2}{147} x^{2}\right) & { }^{5} D_{2} \\
\frac{2^{13 / 2}}{3^{6} 57^{1 / 2}} x^{4} & { }^{5} G_{2}
\end{array}\right. \\
& \mathcal{P}_{L S}^{\left({ }^{3} D_{2} \rightarrow{ }^{3} P_{2}+{ }^{3} S_{1}\right)}=\left\{\begin{array}{cc}
-\frac{2^{5}}{3^{7 / 2}}\left(1-\frac{5}{18} x^{2}+\frac{1}{135} x^{4}\right) & { }^{5} S_{2} \\
-\frac{2^{7 / 2} 5}{3^{11 / 2}} x^{2}\left(1-\frac{2}{75} x^{2}\right) & { }^{3} D_{2} \\
-\frac{2^{7 / 2} 7^{3 / 2}}{3^{11 / 2} 5^{1 / 2}} x^{2}\left(1-\frac{2}{147} x^{2}\right) & { }^{5} D_{2} \\
-\frac{2^{6} 7^{1 / 2}}{3^{11 / 2}} x^{2}\left(1-\frac{4}{105} x^{2}\right) & { }^{7} D_{2} \\
\frac{2^{13 / 2}}{3^{13 / 2} 57^{1 / 2}} x^{4} & { }^{5} G_{2} \\
-\frac{2^{15 / 2}}{3^{13 / 2} 5^{1 / 2} 7^{1 / 2}} x^{4} & { }^{7} G_{2}
\end{array}\right.
\end{aligned}
$$

${ }^{3} \mathrm{D}_{1}$

$$
\begin{aligned}
& \mathcal{P}_{L S}^{\left({ }^{3} D_{1} \rightarrow{ }^{1} P_{1}+{ }^{1} S_{0}\right)}=\left\{\begin{array}{cc}
\frac{2^{6} 5^{1 / 2}}{3^{4}}\left(1-\frac{5}{18} x^{2}+\frac{1}{135} x^{4}\right) & { }^{3} S_{1} \\
\frac{2^{15 / 2}}{3^{6} 5^{1 / 2}} x^{2}\left(1-\frac{1}{6} x^{2}\right) & { }^{3} D_{1}
\end{array}\right. \\
& \mathcal{P}_{L S}^{\left({ }^{3} D_{1} \rightarrow^{3} P_{1}+{ }^{1} S_{0}\right)}=\left\{\begin{array}{cc}
\frac{2^{11 / 2} 5^{1 / 2}}{3^{4}}\left(1-\frac{5}{18} x^{2}+\frac{1}{135} x^{4}\right) & { }^{3} S_{1} \\
\frac{2^{4}(23)}{3^{6} 5^{1 / 2}} x^{2}\left(1+\frac{2}{69} x^{2}\right) & { }^{3} D_{1}
\end{array}\right. \\
& \mathcal{P}_{22}^{\left({ }^{3} D_{1} \rightarrow{ }^{3} P_{2}+{ }^{1} S_{0}\right)}=\frac{2^{4}(13)}{3^{11 / 2} 5^{1 / 2}} x^{2}\left(1-\frac{2}{39} x^{2}\right){ }^{5} D_{1}
\end{aligned}
$$




$$
\begin{aligned}
& \mathcal{P}_{L S}^{\left({ }^{3} D_{1} \rightarrow{ }^{1} P_{1}+{ }^{3} S_{1}\right)}=\left\{\begin{array}{cc}
-\frac{2^{11 / 2} 5^{1 / 2}}{3^{4}}\left(1-\frac{5}{18} x^{2}+\frac{1}{135} x^{4}\right) & { }^{3} S_{1} \\
-\frac{2^{4}(23)}{3^{6} 5^{1 / 2}} x^{2}\left(1+\frac{2}{69} x^{2}\right) & { }^{3} D_{1} \\
-\frac{2^{4}(13)}{3^{11 / 2} 5^{1 / 2}} x^{2}\left(1-\frac{2}{39} x^{2}\right) & { }^{5} D_{1}
\end{array}\right. \\
& \mathcal{P}_{L S}^{\left({ }^{3} D_{1} \rightarrow^{3} P_{0}+{ }^{3} S_{1}\right)}=\left\{\begin{array}{cc}
0 & { }^{3} S_{1} \\
-\frac{2^{9 / 2}(13)}{3^{11 / 2} 5^{1 / 2}} x^{2}\left(1-\frac{2}{39} x^{2}\right) & { }^{3} D_{1}
\end{array}\right. \\
& \mathcal{P}_{L S}^{\left({ }^{3} D_{1} \rightarrow^{3} P_{1}+{ }^{3} S_{1}\right)}=\left\{\begin{array}{cc}
-\frac{2^{5} 5^{1 / 2}}{3^{4}}\left(1-\frac{5}{18} x^{2}+\frac{1}{135} x^{4}\right) & { }^{3} S_{1} \\
-\frac{2^{7 / 2}(47)}{3^{6} 5^{1 / 2}} x^{2}\left(1-\frac{10}{141} x^{2}\right) & { }^{3} D_{1} \\
-\frac{2^{7 / 2}(31)}{3^{11 / 2} 5^{1 / 2}} x^{2}\left(1-\frac{2}{93} x^{2}\right) & { }^{5} D_{1}
\end{array}\right. \\
& \int \frac{2^{5}}{3^{7 / 2}}\left(1-\frac{5}{18} x^{2}+\frac{1}{135} x^{4}\right) \quad{ }^{3} S_{1} \\
& \mathcal{P}_{L S}^{\left({ }^{3} D_{1} \rightarrow{ }^{3} P_{2}+{ }^{3} S_{1}\right)}=\left\{\begin{array}{cc}
-\frac{2^{7 / 2}}{3^{11 / 2}} x^{2}\left(1+\frac{2}{15} x^{2}\right) & { }^{3} D_{1} \\
-\frac{2^{7 / 2}(31)}{3^{6} 5^{1 / 2}} x^{2}\left(1-\frac{2}{93} x^{2}\right) & { }^{5} D_{1}
\end{array}\right. \\
& -\frac{2^{11 / 2} 7^{1 / 2}}{3^{6}} x^{2}\left(1-\frac{4}{105} x^{2}\right) \quad{ }^{7} D_{1} \\
& -\frac{2^{15 / 2}}{3^{11 / 25} 7^{1 / 2}} x^{4} \quad{ }^{7} G_{1}
\end{aligned}
$$

${ }^{1} D_{2}$

$$
\begin{aligned}
& \mathcal{P}_{21}^{\left({ }^{1} D_{2} \rightarrow{ }^{1} P_{1}+{ }^{1} S_{0}\right)}=0 \quad{ }^{3} D_{2} \\
& \mathcal{P}_{20}^{\left({ }^{1} D_{2} \rightarrow{ }^{3} P_{0}+{ }^{1} S_{0}\right)}=-\frac{2^{13 / 2}}{3^{11 / 2} 5^{1 / 2}} x^{2}\left(1-\frac{1}{6} x^{2}\right){ }^{1} D_{2} \\
& \mathcal{P}_{21}^{\left({ }^{1} D_{2} \rightarrow{ }^{3} P_{1}+{ }^{1} S_{0}\right)}=-\frac{2^{9 / 2}}{3^{7 / 2} 5^{1 / 2}} x^{2} \quad{ }^{3} D_{2} \\
& \mathcal{P}_{L S}^{\left({ }^{1} D_{2} \rightarrow{ }^{3} P_{2}+{ }^{1} S_{0}\right)}=\left\{\begin{array}{cc}
-\frac{2^{6}}{3^{7 / 2}}\left(1-\frac{5}{18} x^{2}+\frac{1}{135} x^{4}\right) & { }^{5} S_{2} \\
-\frac{2^{9 / 2} 5^{1 / 2} 7^{1 / 2}}{3^{11 / 2}} x^{2}\left(1-\frac{4}{105} x^{2}\right) & { }^{5} D_{2} \\
-\frac{2^{13 / 2}}{3^{11 / 2} 57^{1 / 2}} x^{4} & { }^{5} G_{2}
\end{array}\right.
\end{aligned}
$$




$$
\begin{aligned}
& \mathcal{P}_{L S}^{\left({ }^{1} D_{2} \rightarrow^{1} P_{1}+^{3} S_{1}\right)}=\left\{\begin{array}{cc}
-\frac{2^{6}}{3^{7 / 2}}\left(1-\frac{5}{18} x^{2}+\frac{1}{135} x^{4}\right) & { }^{5} S_{2} \\
-\frac{2^{13 / 2}}{3^{11 / 2} 5^{1 / 2}} x^{2}\left(1-\frac{1}{6} x^{2}\right) & { }^{1} D_{2} \\
-\frac{2^{9 / 2}}{3^{7 / 2} 5^{1 / 2}} x^{2} & { }^{3} D_{2} \\
-\frac{2^{9 / 2} 5^{1 / 2} 7^{1 / 2}}{3^{11 / 2}} x^{2}\left(1-\frac{4}{105} x^{2}\right) & { }^{5} D_{2} \\
-\frac{2^{13 / 2}}{3^{11 / 25} 7^{1 / 2}} x^{4} & { }^{5} G_{2}
\end{array}\right. \\
& \mathcal{P}_{21}^{\left({ }^{1} D_{2} \rightarrow{ }^{3} P_{0}+{ }^{3} S_{1}\right)}=-\frac{2^{5}}{3^{4} 5^{1 / 2}} x^{2} \quad{ }^{3} D_{2} \\
& \mathcal{P}_{L S}^{\left({ }^{1} D_{2} \rightarrow{ }^{3} P_{1}+{ }^{3} S_{1}\right)}=\left\{\begin{array}{cc}
-\frac{2^{11 / 2}}{3^{7 / 2}}\left(1-\frac{5}{18} x^{2}+\frac{1}{135} x^{4}\right) & { }^{5} S_{2} \\
\frac{2^{7}}{3^{11 / 2} 5^{1 / 2}} x^{2}\left(1-\frac{1}{6} x^{2}\right) & { }^{1} D_{2} \\
\frac{2^{4}}{3^{7 / 2} 5^{1 / 2}} x^{2} & { }^{3} D_{2} \\
-\frac{2^{4} 5^{1 / 2} 7^{1 / 2}}{3^{11 / 2}} x^{2}\left(1-\frac{4}{105} x^{2}\right) & { }^{5} D_{2} \\
-\frac{2^{6}}{3^{11 / 2} 57^{1 / 2}} x^{4} & { }^{5} G_{2}
\end{array}\right. \\
& \mathcal{P}_{L S}^{\left(1 D_{2} \rightarrow^{3} P_{2}+^{3} S_{1}\right)}=\left\{\begin{array}{cc}
\frac{2^{11 / 2}}{3^{3}}\left(1-\frac{5}{18} x^{2}+\frac{1}{135} x^{4}\right) & { }^{5} S_{2} \\
\frac{2^{4}}{3^{4}} x^{2} & { }^{3} D_{2} \\
\frac{2^{4} 5^{1 / 2} 7^{1 / 2}}{3^{5}} x^{2}\left(1-\frac{4}{105} x^{2}\right) & { }^{5} D_{2} \\
0 & { }^{7} D_{2} \\
\frac{2^{6}}{3^{5} 57^{1 / 2}} x^{4} & { }^{5} G_{2} \\
0 & { }^{7} G_{2}
\end{array}\right.
\end{aligned}
$$


$1 F \rightarrow 1 S+1 S$

$$
\begin{aligned}
& f_{D}=-\frac{2^{13 / 2}}{3^{9 / 2} 5^{1 / 2}} x^{2}\left(1-\frac{2}{21} x^{2}\right) \\
& f_{G}=\frac{2^{15 / 2}}{3^{7} 5^{1 / 2} 7^{1 / 2}} x^{4}
\end{aligned}
$$

${ }^{3} \mathbf{F}_{4}$

$$
\begin{aligned}
\mathcal{P}_{40}^{\left({ }^{3} F_{4} \rightarrow{ }^{1} S_{0}+{ }^{1} S_{0}\right)} & =f_{G}{ }^{1} G_{4} \\
\mathcal{P}_{41}^{\left({ }^{3} F_{4} \rightarrow{ }^{3} S_{1}+{ }^{1} S_{0}\right)} & =-\sqrt{\frac{5}{4}} f_{G} \quad{ }^{3} G_{4} \\
\mathcal{P}_{L S}^{\left({ }^{3} F_{4} \rightarrow{ }^{3} S_{1}+{ }^{3} S_{1}\right)} & =\left\{\begin{array}{cc}
f_{D} & { }^{5} D_{4} \\
\sqrt{\frac{1}{3}} f_{G} & { }^{1} G_{4} \\
0 & { }^{3} G_{4} \\
-\sqrt{\frac{55}{42}} f_{G} & { }^{5} G_{4} \\
0 & { }^{5} I_{4}
\end{array}\right.
\end{aligned}
$$

${ }^{3} \mathrm{~F}_{3}$

$$
\begin{aligned}
& \mathcal{P}_{L S}^{\left({ }^{3} F_{3} \rightarrow{ }^{3} S_{1}+{ }^{1} S_{0}\right)}= \begin{cases}-\sqrt{\frac{1}{3}} f_{D} & { }^{3} D_{3} \\
-\sqrt{\frac{27}{28}} f_{G} & { }^{3} G_{3}\end{cases} \\
& \mathcal{P}_{L S}^{\left({ }^{3} F_{3} \rightarrow{ }^{3} S_{1}+{ }^{3} S_{1}\right)}=\left\{\begin{array}{cc}
0 & { }^{3} D_{3} \\
\sqrt{\frac{1}{3}} f_{D} & { }^{5} D_{3} \\
0 & { }^{3} G_{3} \\
-\sqrt{\frac{45}{14}} f_{G} & { }^{5} G_{3}
\end{array}\right.
\end{aligned}
$$

${ }^{3} \mathbf{F}_{2}$ 


$$
\begin{aligned}
\mathcal{P}_{20}^{\left({ }^{3} F_{2} \rightarrow{ }^{1} S_{0}+{ }^{1} S_{0}\right)} & =-\sqrt{\frac{7}{20}} f_{D}{ }^{1} D_{2} \\
\mathcal{P}_{21}^{\left({ }^{3} F_{2} \rightarrow{ }^{3} S_{1}+{ }^{1} S_{0}\right)} & =-\sqrt{\frac{7}{30}} f_{D}{ }^{3} D_{2} \\
\mathcal{P}_{L S}^{\left({ }^{3} F_{2} \rightarrow{ }^{3} S_{1}+{ }^{3} S_{1}\right)} & =\left\{\begin{array}{cc}
0 & { }^{5} S_{2} \\
-\sqrt{\frac{7}{60}} f_{D} & { }^{1} D_{2} \\
0 & { }^{3} D_{2} \\
\sqrt{\frac{1}{15}} f_{D} & { }^{5} D_{2} \\
-\sqrt{\frac{36}{7}} f_{G} & { }^{5} G_{2}
\end{array}\right.
\end{aligned}
$$

${ }^{1} \mathbf{F}_{3}$

$$
\begin{aligned}
& \mathcal{P}_{L S}^{\left({ }^{1} F_{3} \rightarrow{ }^{3} S_{1}+{ }^{1} S_{0}\right)}=\left\{\begin{array}{cc}
\frac{1}{2} f_{D} & { }^{3} D_{3} \\
-\sqrt{\frac{9}{7}} f_{G} & { }^{3} G_{3}
\end{array}\right. \\
& \mathcal{P}_{L S}^{\left({ }^{1} F_{3} \rightarrow{ }^{3} S_{1}+{ }^{3} S_{1}\right)}=\left\{\begin{array}{cc}
-\sqrt{\frac{1}{2}} f_{D} & { }^{3} D_{3} \\
0 & { }^{5} D_{3} \\
\sqrt{\frac{18}{7}} f_{G} & { }^{3} G_{3} \\
0 & { }^{5} G_{3}
\end{array}\right.
\end{aligned}
$$

$$
1 F \rightarrow 2 S+1 S
$$

$$
\begin{aligned}
& f_{D}=-\frac{2^{6}}{3^{5} 5^{1 / 2}} x^{2}\left(1-\frac{13}{42} x^{2}+\frac{1}{189} x^{4}\right) \\
& f_{G}=\frac{2^{10}}{3^{17 / 2} 5^{1 / 2} 7^{1 / 2}} x^{4}\left(1-\frac{1}{48} x^{2}\right)
\end{aligned}
$$

\section{${ }^{3} \mathbf{F}_{4}$}

$$
\begin{aligned}
& \mathcal{P}_{40}^{\left({ }^{3} F_{4} \rightarrow 2^{1} S_{0}+{ }^{1} S_{0}\right)}=f_{G}{ }^{1} G_{4} \\
& \mathcal{P}_{41}^{\left({ }^{3} F_{4} \rightarrow 2^{3} S_{1}+{ }^{1} S_{0}\right)}=-\sqrt{\frac{5}{4}} f_{G} \quad{ }^{3} G_{4}
\end{aligned}
$$




$$
\begin{aligned}
\mathcal{P}_{41}^{\left({ }^{3} F_{4} \rightarrow 2^{1} S_{0}+{ }^{3} S_{1}\right)} & =\sqrt{\frac{5}{4}} f_{G}{ }^{3} G_{4} \\
\mathcal{P}_{L S}^{\left({ }^{3} F_{4} \rightarrow 2^{3} S_{1}+{ }^{3} S_{1}\right)} & =\left\{\begin{array}{cc}
f_{D} & { }^{5} D_{4} \\
\sqrt{\frac{1}{3}} f_{G} & { }^{1} G_{4} \\
0 & { }^{3} G_{4} \\
-\sqrt{\frac{55}{42}} f_{G} & { }^{5} G_{4} \\
0 & { }^{5} I_{4}
\end{array}\right.
\end{aligned}
$$

${ }^{3} \mathbf{F}_{3}$

$$
\begin{aligned}
\mathcal{P}_{L S}^{\left({ }^{3} F_{3} \rightarrow 2^{3} S_{1}+{ }^{1} S_{0}\right)} & =\left\{\begin{array}{cc}
-\sqrt{\frac{1}{3}} f_{D} & { }^{3} D_{3} \\
-\sqrt{\frac{27}{28}} f_{G} & { }^{3} G_{3}
\end{array}\right. \\
\mathcal{P}_{L S}^{\left({ }^{3} F_{3} \rightarrow 2^{1} S_{0}+{ }^{3} S_{1}\right)} & =\left\{\begin{array}{cc}
\sqrt{\frac{1}{3}} f_{D} & { }^{3} D_{3} \\
\sqrt{\frac{27}{28}} f_{G} & { }^{3} G_{3}
\end{array}\right. \\
\mathcal{P}_{L S}^{\left({ }^{3} F_{3} \rightarrow 2^{3} S_{1}+{ }^{3} S_{1}\right)} & =\left\{\begin{array}{cc}
0 & { }^{3} D_{3} \\
\sqrt{\frac{1}{3}} f_{D} & { }^{5} D_{3} \\
0 & { }^{3} G_{3} \\
-\sqrt{\frac{45}{14}} f_{G} & { }^{5} G_{3}
\end{array}\right.
\end{aligned}
$$

${ }^{3} \mathbf{F}_{2}$

$$
\begin{aligned}
& \mathcal{P}_{20}^{\left({ }^{3} F_{2} \rightarrow 2^{1} S_{0}+{ }^{1} S_{0}\right)}=-\sqrt{\frac{7}{20}} f_{D}{ }^{1} D_{2} \\
& \mathcal{P}_{21}^{\left({ }^{3} F_{2} \rightarrow 2^{3} S_{1}+{ }^{1} S_{0}\right)}=-\sqrt{\frac{7}{30}} f_{D}{ }^{3} D_{2} \\
& \mathcal{P}_{21}^{\left({ }^{3} F_{2} \rightarrow 2^{1} S_{0}+{ }^{3} S_{1}\right)}=\sqrt{\frac{7}{30}} f_{D}{ }^{3} D_{2}
\end{aligned}
$$




$$
\mathcal{P}_{L S}^{\left({ }^{3} F_{2} \rightarrow 2^{3} S_{1}+{ }^{3} S_{1}\right)}=\left\{\begin{array}{cc}
0 & { }^{5} S_{2} \\
-\sqrt{\frac{7}{60}} f_{D} & { }^{1} D_{2} \\
0 & { }^{3} D_{2} \\
\sqrt{\frac{1}{15}} f_{D} & { }^{5} D_{2} \\
-\sqrt{\frac{36}{7}} f_{G} & { }^{5} G_{2}
\end{array}\right.
$$

\section{${ }^{1} \mathbf{F}_{3}$}

$$
\begin{aligned}
\mathcal{P}_{L S}^{\left({ }^{1} F_{3} \rightarrow 2^{3} S_{1}+{ }^{1} S_{0}\right)} & =\left\{\begin{array}{cc}
\frac{1}{2} f_{D} & { }^{3} D_{3} \\
-\sqrt{\frac{9}{7}} f_{G} & { }^{3} G_{3}
\end{array}\right. \\
\mathcal{P}_{L S}^{\left({ }^{1} F_{3} \rightarrow 2^{1} S_{0}+{ }^{3} S_{1}\right)} & =\left\{\begin{array}{cc}
\frac{1}{2} f_{D} & { }^{3} D_{3} \\
-\sqrt{\frac{9}{7}} f_{G} & { }^{3} G_{3}
\end{array}\right. \\
\mathcal{P}_{L S}^{\left({ }^{1} F_{3} \rightarrow 2^{3} S_{1}+{ }^{3} S_{1}\right)} & =\left\{\begin{array}{cc}
-\sqrt{\frac{1}{2}} f_{D} & { }^{3} D_{3} \\
0 & { }^{5} D_{3} \\
\sqrt{\frac{18}{7}} f_{G} & { }^{3} G_{3} \\
0 & { }^{5} G_{3}
\end{array}\right.
\end{aligned}
$$

$$
1 F \rightarrow 3 S+1 S
$$

(See $1 F \rightarrow 2 S+1 S$ for channel coefficients.)

$$
\begin{aligned}
& f_{D}=\frac{2^{5}}{3^{5} 5} x^{2}\left(1+x^{2}-\frac{29}{756} x^{4}+\frac{1}{3402} x^{6}\right) \\
& f_{G}=\frac{2^{6} 7^{1 / 2}(11)}{3^{19 / 2} 5} x^{4}\left(1-\frac{10}{231} x^{2}+\frac{1}{2772} x^{4}\right)
\end{aligned}
$$

$$
1 F \rightarrow 1 P+1^{1} S_{0}
$$

${ }^{3} \mathbf{F}_{4}$ 


$$
\begin{aligned}
& \mathcal{P}_{L S}^{\left({ }^{3} F_{4} \rightarrow{ }^{1} P_{1}+{ }^{1} S_{0}\right)}=\left\{\begin{array}{cc}
-\frac{2^{6} 5^{1 / 2}}{3^{6} 7^{1 / 2}} x^{3}\left(1-\frac{4}{135} x^{2}\right) & { }^{3} F_{4} \\
-\frac{2^{7}}{3^{9} 7^{1 / 2}} x^{5} & { }^{3} H_{4}
\end{array}\right. \\
& \mathcal{P}_{L S}^{\left.{ }^{3} F_{4} \rightarrow{ }^{3} P_{1}+{ }^{1} S_{0}\right)}=\left\{\begin{array}{cc}
\frac{2^{15 / 2}}{3^{6} 5^{1 / 2} 7^{1 / 2}} x^{3}\left(1-\frac{5}{108} x^{2}\right) & { }^{3} F_{4} \\
-\frac{2^{13 / 2}}{3^{9} 7^{1 / 2}} x^{5} & { }^{3} H_{4}
\end{array}\right. \\
& \mathcal{P}_{L S}^{\left.{ }^{3} F_{4} \rightarrow{ }^{3} P_{2}+{ }^{1} S_{0}\right)}=\left\{\begin{array}{cc}
\frac{2^{13 / 2}}{3^{11 / 2} 7^{1 / 2}} x^{3}\left(1-\frac{1}{54} x^{2}\right) & { }^{5} F_{4} \\
\frac{2^{6}}{3^{17 / 2} 7^{1 / 2}} x^{5} & { }^{5} H_{4}
\end{array}\right.
\end{aligned}
$$

${ }^{3} \mathbf{F}_{3}$

$$
\begin{aligned}
& \mathcal{P}_{31}^{\left({ }^{3} F_{3} \rightarrow^{1} P_{1}+{ }^{1} S_{0}\right)}=\frac{2^{6}}{3^{5} 5^{1 / 2} 7^{1 / 2}} x^{3} \quad{ }^{3} F_{3} \\
& \mathcal{P}_{30}^{\left({ }^{3} F_{3} \rightarrow{ }^{3} P_{0}+{ }^{1} S_{0}\right)}=\frac{2^{6}}{3^{5} 5^{1 / 2} 7^{1 / 2}} x^{3} \quad{ }^{1} F_{3} \\
& \mathcal{P}_{31}^{\left({ }^{3} F_{3} \rightarrow{ }^{3} P_{1}+{ }^{1} S_{0}\right)}=\frac{2^{13 / 2}}{3^{5} 5^{1 / 2} 7^{1 / 2}} x^{3}\left(1-\frac{1}{18} x^{2}\right) \quad{ }^{3} F_{3} \\
& \mathcal{P}_{L S}^{\left({ }^{3} F_{3} \rightarrow{ }^{3} P_{2}+{ }^{1} S_{0}\right)}=\left\{\begin{array}{cc}
-\frac{2^{15 / 2}}{3^{5}} x\left(1-\frac{1}{6} x^{2}+\frac{1}{315} x^{4}\right) & { }^{5} P_{3} \\
\frac{2^{11 / 2}}{3^{15 / 25} 7^{1 / 2}} x^{5} & { }^{5} F_{3} \\
\frac{2^{6}}{3^{15 / 27}} x^{5} & { }^{5} H_{3}
\end{array}\right.
\end{aligned}
$$

${ }^{3} \mathbf{F}_{2}$

$$
\begin{aligned}
& \mathcal{P}_{L S}^{\left({ }^{3} F_{2} \rightarrow^{1} P_{1}+{ }^{1} S_{0}\right)}=\left\{\begin{array}{cc}
-\frac{2^{13 / 2} 7^{1 / 2}}{3^{9 / 2} 5^{1 / 2}} x\left(1-\frac{1}{6} x^{2}+\frac{1}{315} x^{4}\right) & { }^{3} P_{2} \\
-\frac{2^{5}}{3^{5} 5^{1 / 2} 7^{1 / 2}} x^{3}\left(1-\frac{2}{15} x^{2}\right) & { }^{3} F_{2}
\end{array}\right. \\
& \mathcal{P}_{L S}^{\left({ }^{3} F_{2} \rightarrow^{3} P_{1}+{ }^{1} S_{0}\right)}=\left\{\begin{array}{cc}
-\frac{2^{6} 7^{1 / 2}}{3^{9 / 2} 5^{1 / 2}} x\left(1-\frac{1}{6} x^{2}+\frac{1}{315} x^{4}\right) & { }^{3} P_{2} \\
-\frac{2^{11 / 2}}{3^{5} 5^{1 / 2} 7^{1 / 2}} x^{3}\left(1+\frac{2}{45} x^{2}\right) & { }^{3} F_{2}
\end{array}\right. \\
& \mathcal{P}_{L S}^{\left({ }^{3} F_{2} \rightarrow{ }^{3} P_{2}+{ }^{1} S_{0}\right)}=\left\{\begin{array}{cc}
-\frac{2^{6} 7^{1 / 2}}{3^{5} 5^{1 / 2}} x\left(1-\frac{1}{6} x^{2}+\frac{1}{315} x^{4}\right) & { }^{5} P_{2} \\
-\frac{2^{6}}{3^{5} 5^{1 / 2} 7^{1 / 2}} x^{3}\left(1-\frac{2}{45} x^{2}\right) & { }^{5} F_{2}
\end{array}\right.
\end{aligned}
$$

${ }^{1} \mathbf{F}_{3}$ 


$$
\begin{aligned}
& \mathcal{P}_{31}^{\left({ }^{1} F_{3} \rightarrow{ }^{1} P_{1}+{ }^{1} S_{0}\right)}=0 \quad{ }^{3} F_{3} \\
& \mathcal{P}_{30}^{\left({ }^{1} F_{3} \rightarrow{ }^{3} P_{0}+{ }^{1} S_{0}\right)}=\frac{2^{5}}{3^{9 / 2} 5^{1 / 2} 7^{1 / 2}} x^{3}\left(1-\frac{2}{27} x^{2}\right){ }^{1} F_{3} \\
& \mathcal{P}_{31}^{\left({ }^{1} F_{3} \rightarrow{ }^{3} P_{1}+{ }^{1} S_{0}\right)}=\frac{2^{11 / 2}}{3^{9 / 2} 5^{1 / 2} 7^{1 / 2}} x^{3} \quad{ }^{3} F_{3} \\
& \mathcal{P}_{L S}^{\left({ }^{1} F_{3} \rightarrow{ }^{3} P_{2}+{ }^{1} S_{0}\right)}=\left\{\begin{array}{cc}
\frac{2^{13 / 2}}{3^{9 / 2}} x\left(1-\frac{1}{6} x^{2}+\frac{1}{315} x^{4}\right) & { }^{5} P_{3} \\
\frac{2^{11 / 2}}{3^{5} 7^{1 / 2}} x^{3}\left(1-\frac{4}{135} x^{2}\right) & { }^{5} F_{3} \\
\frac{2^{7}}{3^{87}} x^{5} & { }^{5} H_{3}
\end{array}\right.
\end{aligned}
$$

$1 F \rightarrow 2 P+1^{1} S_{0}$

\section{${ }^{3} \mathbf{F}_{4}$}

$$
\begin{aligned}
& \mathcal{P}_{L S}^{\left({ }^{3} F_{4} \rightarrow 2^{1} P_{1}+{ }^{1} S_{0}\right)}=\left\{\begin{array}{cc}
-\frac{2^{11 / 2}(37)}{3^{7} 57^{1 / 2}} x^{3}\left(1-\frac{125}{1998} x^{2}+\frac{2}{2997} x^{4}\right) & { }^{3} F_{4} \\
-\frac{2^{15 / 2} 5^{1 / 2}}{3^{10} 7^{1 / 2}} x^{5}\left(1-\frac{1}{60} x^{2}\right) & { }^{3} H_{4}
\end{array}\right. \\
& \mathcal{P}_{L S}^{\left({ }^{3} F_{4} \rightarrow 2^{3} P_{1}+{ }^{1} S_{0}\right)}=\left\{\begin{array}{cc}
\frac{2^{6}(13)}{3^{7} 57^{1 / 2}} x^{3}\left(1-\frac{34}{351} x^{2}+\frac{5}{4212} x^{4}\right) & { }^{3} F_{4} \\
-\frac{2^{7} 5^{1 / 2}}{3^{10} 7^{1 / 2}} x^{5}\left(1-\frac{1}{60} x^{2}\right) & { }^{3} H_{4}
\end{array}\right. \\
& \mathcal{P}_{L S}^{\left({ }^{3} F_{4} \rightarrow 2^{3} P_{2}+{ }^{1} S_{0}\right)}=\left\{\begin{array}{cc}
\frac{2^{9}}{3^{13 / 2} 5^{1 / 2} 7^{1 / 2}} x^{3}\left(1-\frac{19}{432} x^{2}+\frac{1}{2592} x^{4}\right) & { }^{5} F_{4} \\
\frac{2^{13 / 2} 5^{1 / 2}}{3^{19 / 2} 7^{1 / 2}} x^{5}\left(1-\frac{1}{60} x^{2}\right) & { }^{5} H_{4}
\end{array}\right.
\end{aligned}
$$

${ }^{3} \mathbf{F}_{3}$

$$
\begin{aligned}
& \mathcal{P}_{31}^{\left({ }^{3} F_{3} \rightarrow 2^{1} P_{1}+{ }^{1} S_{0}\right)}=\frac{2^{11 / 2}}{3^{4} 57^{1 / 2}} x^{3}\left(1-\frac{1}{54} x^{2}\right){ }^{3} F_{3} \\
& \mathcal{P}_{30}^{\left({ }^{3} F_{3} \rightarrow 2^{3} P_{0}+{ }^{1} S_{0}\right)}=\frac{2^{11 / 2}}{3^{4} 57^{1 / 2}} x^{3}\left(1-\frac{1}{54} x^{2}\right){ }^{1} F_{3} \\
& \mathcal{P}_{31}^{\left({ }^{3} F_{3} \rightarrow 2^{3} P_{1}+{ }^{1} S_{0}\right)}=\frac{2^{7}}{3^{5} 57^{1 / 2}} x^{3}\left(1-\frac{13}{108} x^{2}+\frac{1}{648} x^{4}\right){ }^{3} F_{3}
\end{aligned}
$$




$$
\mathcal{P}_{L S}^{\left({ }^{3} F_{3} \rightarrow 2^{3} P_{2}+{ }^{1} S_{0}\right)}=\left\{\begin{array}{cc}
-\frac{2^{7}}{3^{5} 5^{1 / 2}} x\left(1-\frac{7}{15} x^{2}+\frac{5}{252} x^{4}-\frac{1}{5670} x^{6}\right) & { }^{5} P_{3} \\
\frac{2^{6}}{3^{11 / 2} 5^{3 / 2} 7^{1 / 2}} x^{3}\left(1+\frac{5}{27} x^{2}-\frac{1}{324} x^{4}\right) & { }^{5} F_{3} \\
\frac{2^{13 / 2} 5^{1 / 2}}{3^{17 / 2} 7} x^{5}\left(1-\frac{1}{60} x^{2}\right) & { }^{5} H_{3}
\end{array}\right.
$$

${ }^{3} \mathrm{~F}_{2}$

$$
\begin{aligned}
& \mathcal{P}_{L S}^{\left({ }^{3} F_{2} \rightarrow 2^{1} P_{1}+{ }^{1} S_{0}\right)}=\left\{\begin{array}{cc}
-\frac{2^{6} 7^{1 / 2}}{3^{9 / 2} 5} x\left(1-\frac{7}{15} x^{2}+\frac{5}{252} x^{4}-\frac{1}{5670} x^{6}\right) & { }^{3} P_{2} \\
-\frac{2^{9 / 2}}{3^{4} 5^{2} 7^{1 / 2}} x^{3}\left(1-\frac{5}{6} x^{2}+\frac{1}{81} x^{4}\right) & { }^{3} F_{2}
\end{array}\right. \\
& \mathcal{P}_{L S}^{\left({ }^{3} F_{2} \rightarrow 2^{3} P_{1}+{ }^{1} S_{0}\right)}=\left\{\begin{array}{cc}
-\frac{2^{11 / 2} 7^{1 / 2}}{3^{9 / 2} 5} x\left(1-\frac{7}{15} x^{2}+\frac{5}{252} x^{4}-\frac{1}{5670} x^{6}\right) & { }^{3} P_{2} \\
-\frac{2^{5}(19)}{3^{5} 5^{2} 7^{1 / 2}} x^{3}\left(1+\frac{25}{1026} x^{2}-\frac{1}{1539} x^{4}\right) & { }^{3} F_{2}
\end{array}\right. \\
& \mathcal{P}_{L S}^{\left({ }^{3} F_{2} \rightarrow 2^{3} P_{2}+{ }^{1} S_{0}\right)}=\left\{\begin{array}{cc}
-\frac{2^{11 / 2} 7^{1 / 2}}{3^{5} 5} x\left(1-\frac{7}{15} x^{2}+\frac{5}{252} x^{4}-\frac{1}{5670} x^{6}\right) & { }^{5} P_{2} \\
-\frac{2^{11 / 2}(11)}{3^{5} 5^{2} 7^{1 / 2}} x^{3}\left(1-\frac{5}{54} x^{2}+\frac{1}{891} x^{4}\right) & { }^{5} F_{2}
\end{array}\right.
\end{aligned}
$$

\section{${ }^{1} F_{3}$}

$$
\begin{aligned}
& \mathcal{P}_{31}^{\left({ }^{1} F_{3} \rightarrow 2^{1} P_{1}+{ }^{1} S_{0}\right)}=0{ }^{3} F_{3} \\
& \mathcal{P}_{30}^{\left({ }^{1} F_{3} \rightarrow 2^{3} P_{0}+{ }^{1} S_{0}\right)}=\frac{2^{9 / 2}}{3^{11 / 2} 7^{1 / 2}} x^{3}\left(1-\frac{49}{270} x^{2}+\frac{1}{405} x^{4}\right){ }^{1} F_{3} \\
& \mathcal{P}_{31}^{\left({ }^{1} F_{3} \rightarrow 2^{3} P_{1}+{ }^{1} S_{0}\right)}=\frac{2^{5}}{\frac{3^{7 / 2} 57^{1 / 2}}{3}\left(1-\frac{1}{54} x^{2}\right) \quad{ }^{3} F_{3}} \\
& \mathcal{P}_{L S}^{\left({ }^{1} F_{3} \rightarrow 2^{3} P_{2}+{ }^{1} S_{0}\right)}=\left\{\begin{array}{cc}
\frac{2^{6}}{3^{9 / 2} 5^{1 / 2}} x\left(1-\frac{7}{15} x^{2}+\frac{5}{252} x^{4}-\frac{1}{5670} x^{6}\right) & { }^{5} P_{3} \\
\frac{2^{5}(37)}{3^{6} 5^{3 / 2} 7^{1 / 2}} x^{3}\left(1-\frac{125}{1998} x^{2}+\frac{2}{2997} x^{4}\right) & { }^{5} F_{3} \\
\frac{2^{15 / 2} 5^{1 / 2}}{3^{9} 7} x^{5}\left(1-\frac{1}{60} x^{2}\right) & { }^{5} H_{3}
\end{array}\right.
\end{aligned}
$$

$1 F \rightarrow 1 D+1^{1} S_{0}$

${ }^{3} \mathbf{F}_{4}$ 


$$
\begin{aligned}
& \mathcal{P}_{L S}^{\left({ }^{3} F_{4} \rightarrow{ }^{1} D_{2}+{ }^{1} S_{0}\right)}=\left\{\begin{array}{cc}
\frac{2^{7}}{3^{11 / 25^{1 / 2}}} x^{2}\left(1-\frac{5}{42} x^{2}+\frac{1}{567} x^{4}\right) & { }^{5} D_{4} \\
\frac{2^{11 / 2} 5(11)^{1 / 2}}{3^{17 / 27}} x^{4}\left(1-\frac{4}{165} x^{2}\right) & { }^{5} G_{4} \\
\frac{2^{13 / 2}}{3^{19 / 2} 7^{1 / 2}(11)^{1 / 2}} x^{6} & { }^{5} I_{4}
\end{array}\right. \\
& \mathcal{P}_{41}^{\left({ }^{3} F_{4} \rightarrow{ }^{3} D_{1}+{ }^{1} S_{0}\right)}=\frac{2^{5} 7^{1 / 2}}{3^{17 / 2} 5^{1 / 2}} x^{4}\left(1-\frac{1}{21} x^{2}\right) \quad{ }^{3} G_{4} \\
& \mathcal{P}_{L S}^{\left({ }^{3} F_{4} \rightarrow{ }^{3} D_{2}+{ }^{1} S_{0}\right)}=\left\{\begin{array}{cc}
-\frac{2^{13 / 2}}{3^{6} 5^{1 / 2}} x^{2}\left(1-\frac{11}{42} x^{2}+\frac{5}{1134} x^{4}\right) & { }^{5} D_{4} \\
\frac{2^{5}(11)^{1 / 2}}{3^{87}} x^{4}\left(1+\frac{1}{33} x^{2}\right) & { }^{5} G_{4} \\
\frac{2^{7}}{3^{10} 7^{1 / 2}(11)^{1 / 2}} x^{6} & { }^{5} I_{4}
\end{array}\right. \\
& \mathcal{P}_{L S}^{\left({ }^{3} F_{4} \rightarrow{ }^{3} D_{3}+{ }^{1} S_{0}\right)}=\left\{\begin{array}{cc}
-\frac{2^{15 / 2}}{3^{6}} x^{2}\left(1-\frac{1}{21} x^{2}+\frac{1}{2268} x^{4}\right) & { }^{7} D_{4} \\
-\frac{2^{7}(11)^{1 / 2}}{3^{75^{1 / 2}}} x^{4}\left(1-\frac{1}{66} x^{2}\right) & { }^{7} G_{4} \\
-\frac{2^{11 / 2}}{3^{10}(11)^{1 / 2}} x^{6} & { }^{7} I_{4}
\end{array}\right.
\end{aligned}
$$

${ }^{3} \mathbf{F}_{2}$ 


$$
\begin{aligned}
& \mathcal{P}_{L S}^{\left.{ }^{3} F_{2} \rightarrow^{1} D_{2}+{ }^{1} S_{0}\right)}=\left\{\begin{array}{cc}
\frac{2^{13 / 2} 7^{1 / 2}}{3^{4} 5^{1 / 2}}\left(1-\frac{1}{3} x^{2}+\frac{1}{60} x^{4}-\frac{1}{5670} x^{6}\right) & { }^{5} S_{2} \\
\frac{2^{6}}{3^{4} 5} x^{2}\left(1-\frac{5}{42} x^{2}+\frac{1}{567} x^{4}\right) & { }^{5} D_{2} \\
\frac{2^{5}}{3^{5} 5^{3 / 2} 7} x^{4}\left(1-\frac{2}{9} x^{2}\right) & { }^{5} G_{2}
\end{array}\right. \\
& \mathcal{P}_{21}^{\left({ }^{3} F_{2} \rightarrow{ }^{3} D_{1}+{ }^{1} S_{0}\right)}=\frac{2^{11 / 2} 7^{1 / 2}}{3^{11 / 2} 5^{1 / 2}} x^{2}\left(1+\frac{1}{30} x^{2}-\frac{1}{945} x^{4}\right) \quad{ }^{3} D_{2} \\
& \mathcal{P}_{L S}^{\left({ }^{3} F_{2} \rightarrow{ }^{3} D_{2}+{ }^{1} S_{0}\right)}=\left\{\begin{array}{cc}
\frac{2^{7} 7^{1 / 2}}{3^{9 / 2} 5^{1 / 2}}\left(1-\frac{1}{3} x^{2}+\frac{1}{60} x^{4}-\frac{1}{5670} x^{6}\right) & { }^{5} S_{2} \\
\frac{2^{11 / 2}(11)}{3^{11 / 25}} x^{2}\left(1-\frac{23}{462} x^{2}+\frac{1}{2079} x^{4}\right) & { }^{5} D_{2} \\
\frac{2^{15 / 2}(11)}{3^{15 / 2} 5^{3 / 2} 7} x^{4}\left(1+\frac{1}{33} x^{2}\right) & { }^{5} G_{2}
\end{array}\right. \\
& \mathcal{P}_{L S}^{\left({ }^{3} F_{2} \rightarrow{ }^{3} D_{3}+{ }^{1} S_{0}\right)}=\left\{\begin{array}{cc}
\frac{2^{7}}{3^{11 / 2} 5^{1 / 2}} x^{2}\left(1-\frac{17}{210} x^{2}+\frac{1}{845} x^{4}\right) & { }^{7} D_{2} \\
\frac{2^{11 / 2}(23)}{3^{15 / 2} 57} x^{4}\left(1-\frac{2}{69} x^{2}\right) & { }^{7} G_{2}
\end{array}\right.
\end{aligned}
$$

${ }^{1} F_{3}$

$$
\begin{aligned}
& \mathcal{P}_{L S}^{\left({ }^{1} F_{3} \rightarrow{ }^{1} D_{2}+{ }^{1} S_{0}\right)}= \begin{cases}0 & { }^{5} D_{3} \\
0 & { }^{5} G_{3}\end{cases} \\
& \mathcal{P}_{L S}^{\left({ }^{1} F_{3} \rightarrow{ }^{3} D_{1}+{ }^{1} S_{0}\right)}=\left\{\begin{array}{cc}
-\frac{2^{6}}{3^{55}} x^{2}\left(1-\frac{13}{42} x^{2}+\frac{1}{189} x^{4}\right) & { }^{3} D_{3} \\
-\frac{2^{5}}{3^{15 / 2} 57} x^{4}\left(1-\frac{4}{3} x^{2}\right) & { }^{3} G_{3}
\end{array}\right. \\
& \mathcal{P}_{L S}^{\left({ }^{1} F_{3} \rightarrow{ }^{3} D_{2}+{ }^{1} S_{0}\right)}=\left\{\begin{array}{cc}
-\frac{2^{13 / 2}}{3^{5} 5^{1 / 2}} x^{2}\left(1-\frac{1}{42} x^{2}\right) & { }^{5} D_{3} \\
-\frac{2^{5}}{3^{67}} x^{4} & { }^{5} G_{3}
\end{array}\right. \\
& \mathcal{P}_{L S}^{\left({ }^{1} F_{3} \rightarrow{ }^{3} D_{3}+{ }^{1} S_{0}\right)}=\left\{\begin{array}{cc}
-\frac{2^{13 / 2}}{3^{4}}\left(1-\frac{1}{3} x^{2}+\frac{1}{60} x^{4}-\frac{1}{5670} x^{6}\right) & { }^{7} S_{3} \\
-\frac{2^{15 / 2}}{3^{9 / 2}} x^{2}\left(1-\frac{1}{14} x^{2}+\frac{1}{1134} x^{4}\right) & { }^{7} D_{3} \\
-\frac{2^{5}(11)^{1 / 2}}{3^{5} 57} x^{4}\left(1-\frac{2}{99} x^{2}\right) & { }^{7} G_{3} \\
-\frac{2^{13 / 2}}{3^{17 / 2} 7(11)^{1 / 2}} x^{6} & { }^{7} I_{3}
\end{array}\right.
\end{aligned}
$$




\section{APPENDIX B: NUMERICAL DECAY RATES.}

In this appendix we quote numerical values for partial widths predicted by the ${ }^{3} \mathrm{P}_{0}$ model. The masses used are experimental values of well established candidates, usually taken from the $1996 \mathrm{PDG}$, otherwise we used an approximate multiplet mass. These are $1700 \mathrm{MeV}$ (2P), $1670 \mathrm{MeV}$ (1D), $2050 \mathrm{MeV}$ (1F), and $1900 \mathrm{MeV}$ and $1800 \mathrm{MeV}$ respectively for the $3^{3} \mathrm{~S}_{1}$ and $3^{1} \mathrm{~S}_{0}$. The lighter meson masses assumed are $m_{\pi}=138 \mathrm{MeV}$, $m_{K}=496 \mathrm{MeV}, m_{\rho}=770 \mathrm{MeV}, m_{\omega}=782 \mathrm{MeV}$ and $m_{K^{*}}=894 \mathrm{MeV}$. For other states we used the 1996 PDG masses except for the broad $f_{0}$, which we left at $1300 \mathrm{MeV}$.

Although we found optimum parameters near $\gamma=0.5$ and $\beta=0.4 \mathrm{GeV}$ in a fit to light 1S and 1P decays, these parameters lead to moderate overestimates of the widths of the well established higher-L states $\pi_{2}(1670)$ and $f_{4}(2044)$; with this $\beta$ a value closer to $\gamma=0.4$ is preferred. Consequently we quote widths for all these higher quarkonia with the parameters

$$
(\gamma, \beta)=(0.4,0.4 \mathrm{GeV})
$$

The tables are largely self explanatory. Except in a few cases the states are specified uniquely by their labels. The exceptions include the $|\eta(547)\rangle$ and $\left|\eta^{\prime}(958)\right\rangle$, which we take to be the usual $1 / \sqrt{2}$ combinations of $|n \bar{n}\rangle$ and $|s \bar{s}\rangle$ basis states. We assume that the $|\eta(1295)\rangle$ and $\left|\eta_{2}(1645)\right\rangle$ are pure $|n \bar{n}\rangle$ states. The strange mesons $K_{1}(1273)$ and $K_{1}(1402)$ are taken to be the linear combinations

$$
\left|K_{1}(1273)\right\rangle=\sqrt{\frac{2}{3}}\left|{ }^{1} P_{1}\right\rangle+\sqrt{\frac{1}{3}}\left|{ }^{3} P_{1}\right\rangle
$$

and

$$
\left|K_{1}(1402)\right\rangle=-\sqrt{\frac{1}{3}}\left|{ }^{1} P_{1}\right\rangle+\sqrt{\frac{2}{3}}\left|{ }^{3} P_{1}\right\rangle
$$

This gives a zero S-wave $K_{1}(1273) \rightarrow K^{*} \pi$ coupling; experimentally $\mathrm{D} / \mathrm{S}=1.0(0.7)$, and the small partial width implies a small S-wave amplitude. The orthogonal state $K_{1}(1402)$ 
(B3) is predicted to have a $\mathrm{D} / \mathrm{S}$ ratio of +0.049 in $K^{*} \pi$, quite close to the experimental $\mathrm{D} / \mathrm{S}=+0.04(1)$. The large $K_{1}(1273) \rightarrow K \rho$ mode is not predicted and is possibly due to a virtual intermediate state such as $\mathrm{K}_{0}^{*}(1429) \pi$ followed by a final-state interaction.

The tables give partial widths for all nonstrange 2S, 3S, 2P, 1D and $1 \mathrm{~F}$ quarkonia to all two-body modes allowed by phase space, rounded to the nearest $\mathrm{MeV}$. The predictions of the dominant modes of the "missing states" in the quark model, such as the $2^{--}$states and most of the $1 \mathrm{~F}$ states, are especially interesting. If the ${ }^{3} \mathrm{P}_{0}$ model has even moderate accuracy these tables should be very useful in searches for these states. 


\begin{tabular}{|c|c|c|c|}
\hline \multicolumn{4}{|c|}{ Table B1. Partial widths of $2^{3} \mathrm{~S}_{1}$ states $(\mathrm{MeV})$. } \\
\hline Mode & $\rho(1465)$ & Mode & $\omega(1419)$ \\
\hline \multicolumn{4}{|c|}{$(1 \mathrm{~S})^{2}$} \\
\hline$\pi \pi$ & 74. & & \\
\hline$\omega \pi$ & 122. & $\rho \pi$ & 328. \\
\hline$\rho \eta$ & 25. & $\omega \eta$ & 12. \\
\hline \multicolumn{4}{|c|}{$(2 S)(1 S)$} \\
\hline$\pi(1300) \pi$ & 0. & & \\
\hline \multicolumn{4}{|c|}{$(\mathbf{1 P})(\mathbf{1 S})$} \\
\hline$h_{1}(1170) \pi$ & 1. & $b_{1}(1231) \pi$ & 1. \\
\hline$a_{1}(1230) \pi$ & 3. & & \\
\hline$a_{2}(1318) \pi$ & 0. & & \\
\hline \multicolumn{4}{|c|}{$(1 S)^{2}$ strange } \\
\hline$K K$ & 35. & & 31. \\
\hline$K^{*} K$ & 19. & & 5. \\
\hline \multicolumn{4}{|c|}{ total } \\
\hline$\sum_{i} \Gamma_{i}$ & 279. & & 378. \\
\hline$\Gamma_{\text {expt }}$ & $310(60)$ & & $174(59)$ \\
\hline
\end{tabular}




\begin{tabular}{||c|c||c|c||}
\hline \multicolumn{4}{||c||}{ Table B2. Partial widths of $2^{1} \mathrm{~S}_{0}$ states $(\mathrm{MeV})}$. \\
\hline \hline Mode & $\pi(1300)$ & Mode & $\eta(1295)$ \\
\hline \hline \multicolumn{4}{|c||}{$(1 \mathrm{~S})^{2}$} \\
\hline$\pi \rho$ & 209. & none open & \\
\hline \hline \multicolumn{4}{|c||}{ total } \\
\hline$\sum_{i} \Gamma_{i}$ & 209. & & 0. \\
\hline$\Gamma_{\text {expt }}$ & $200-600$ & & $53(6)$ \\
\hline
\end{tabular}




\begin{tabular}{|c|c|c|c|}
\hline \multicolumn{4}{|c|}{ Table B3. Partial widths of $3^{3} \mathrm{~S}_{1}$ states $(\mathrm{MeV})$. } \\
\hline Mode & $\rho(1900)$ & Mode & $\omega(1900)$ \\
\hline \multicolumn{4}{|c|}{$(\mathbf{1 S})^{2}$} \\
\hline$\pi \pi$ & 1. & & \\
\hline$\omega \pi$ & 5. & $\rho \pi$ & 14. \\
\hline$\rho \eta$ & 8. & $\omega \eta$ & 8. \\
\hline$\rho \eta^{\prime}$ & 11. & $\omega \eta^{\prime}$ & 10. \\
\hline$\rho \rho$ & 92. & & \\
\hline \multicolumn{4}{|c|}{$(2 S)(1 S)$} \\
\hline$\pi(1300) \pi$ & 70. & & \\
\hline$\omega(1419) \pi$ & 50. & $\rho(1465) \pi$ & 121. \\
\hline \multicolumn{4}{|c|}{$(1 \mathrm{P})(1 \mathrm{~S})$} \\
\hline$h_{1}(1170) \pi$ & 32. & $b_{1}(1231) \pi$ & 75. \\
\hline$b_{1}(1231) \eta$ & 4. & $h_{1}(1170) \eta$ & 6. \\
\hline$a_{1}(1230) \pi$ & 26. & & \\
\hline$a_{2}(1318) \pi$ & 46. & & \\
\hline \multicolumn{4}{|c|}{$(2 \mathrm{P})(1 \mathrm{~S})$} \\
\hline$h_{1}(1700) \pi$ & 0 . & $b_{1}(1700) \pi$ & 0. \\
\hline$a_{1}(1700) \pi$ & 0. & & \\
\hline$a_{2}(1700) \pi$ & 0. & & \\
\hline \multicolumn{4}{|c|}{$(1 D)(1 S)$} \\
\hline$\pi_{2}(1670) \pi$ & 0. & & \\
\hline$\omega_{1}(1649) \pi$ & 0. & $\rho_{1}(1700) \pi$ & 0. \\
\hline$\omega_{2}(1670) \pi$ & 0. & $\rho_{2}(1670) \pi$ & 0. \\
\hline$\omega_{3}(1667) \pi$ & 0. & $\rho_{3}(1691) \pi$ & 0. \\
\hline
\end{tabular}


Table B3 (cont.) Partial widths of $3^{3} \mathrm{~S}_{1}$ states $(\mathrm{MeV})$.

\begin{tabular}{|c|c|c|c|}
\hline Mode & $\rho(1900)$ & Mode & $\omega(1900)$ \\
\hline \multicolumn{4}{|c|}{$(1 S)^{2}$ strange } \\
\hline$K K$ & 1. & & 1. \\
\hline$K^{*} K$ & 21. & & 21. \\
\hline$K^{*} K^{*}$ & 27. & & 27. \\
\hline \multicolumn{4}{|c|}{$(1 \mathrm{P})(1 \mathrm{~S})$ strange } \\
\hline$K_{1}^{*}(1273) K$ & 5. & & 5. \\
\hline$K_{1}^{*}(1402) K$ & 4. & & 4. \\
\hline \multicolumn{4}{|c|}{ total } \\
\hline$\sum_{i} \Gamma_{i}$ & 403. & & 292. \\
\hline
\end{tabular}




\begin{tabular}{|c|c|c|c|}
\hline \multicolumn{4}{|c|}{ Table B4. Partial widths of $3^{1} \mathrm{~S}_{0}$ states $(\mathrm{MeV})$} \\
\hline Mode & $\pi(1800)$ & Mode & $\eta(1800)$ \\
\hline \multicolumn{4}{|c|}{$(\mathbf{1 S})^{2}$} \\
\hline$\pi \rho$ & 31. & & \\
\hline \multirow[t]{2}{*}{$\rho \omega$} & 73. & $\rho \rho$ & 112. \\
\hline & & $\omega \omega$ & 36. \\
\hline \multicolumn{4}{|c|}{$(2 S)(1 S)$} \\
\hline$\rho(1465) \pi$ & 53. & & \\
\hline \multicolumn{4}{|c|}{$(1 \mathrm{P})(1 \mathrm{~S})$} \\
\hline$f_{0}(1300) \pi$ & 7. & $a_{0}(1450) \pi$ & 30. \\
\hline$f_{2}(1275) \pi$ & 28. & $a_{2}(1318) \pi$ & 61. \\
\hline \multicolumn{4}{|c|}{$(1 \mathrm{~S})^{2}$ strange } \\
\hline$K^{*} K$ & 36. & & 36. \\
\hline \multicolumn{4}{|c|}{ total } \\
\hline$\sum_{i} \Gamma_{i}$ & 228. & & 275. \\
\hline$\Gamma_{\text {expt }}$ & $212(37)$ & & \\
\hline
\end{tabular}




\begin{tabular}{|c|c|c|c|}
\hline \multicolumn{4}{|c|}{ Table B5. Partial widths of $2{ }^{3} \mathrm{P}_{J} a_{J}$ states $(\mathrm{MeV})$} \\
\hline Mode & $a_{2}(1700)$ & $a_{1}(1700)$ & $a_{0}(1700)$ \\
\hline \multicolumn{4}{|c|}{$(\mathbf{1 S})^{2}$} \\
\hline$\eta \pi$ & 23. & & 5. \\
\hline$\eta^{\prime} \pi$ & 10. & & 5. \\
\hline$\rho \pi$ & 104. & 58. & \\
\hline$\omega \rho$ & 109. & 15. & 46. \\
\hline \multicolumn{4}{|c|}{$(2 S)(1 S)$} \\
\hline$\eta(1295) \pi$ & 3. & & 43. \\
\hline$\rho(1465) \pi$ & 0. & 41. & \\
\hline \multicolumn{4}{|c|}{$(\mathbf{1 P})(\mathbf{1 S})$} \\
\hline$b_{1}(1231) \pi$ & 28. & 41. & 165. \\
\hline$f_{0}(1300) \pi$ & & 2. & \\
\hline$f_{1}(1282) \pi$ & 4. & 18. & 30. \\
\hline$f_{2}(1275) \pi$ & 20. & 39. & \\
\hline \multicolumn{4}{|c|}{$(1 \mathrm{~S})^{2}$ strange } \\
\hline$K K$ & 20. & & 0. \\
\hline$K^{*} K$ & 17. & 33. & \\
\hline \multicolumn{4}{|c|}{ total } \\
\hline$\sum_{i} \Gamma_{i}$ & 336. & 246. & 293. \\
\hline
\end{tabular}




\begin{tabular}{|c|c|c|c|}
\hline \multicolumn{4}{|c|}{ Table B6. Partial widths of $2{ }^{3} \mathrm{P}_{J} f_{J}$ states $(\mathrm{MeV})$. } \\
\hline Mode & $f_{2}(1700)$ & $f_{1}(1700)$ & $f_{0}(1700)$ \\
\hline \multicolumn{4}{|c|}{$(1 \mathrm{~S})^{2}$} \\
\hline$\pi \pi$ & 81. & & 47. \\
\hline$\eta \eta$ & 4. & & 0. \\
\hline$\eta \eta^{\prime}$ & 1. & & 16. \\
\hline$\rho \rho$ & 159. & 27. & 72. \\
\hline$\omega \omega$ & 56. & 6. & 22. \\
\hline \multicolumn{4}{|c|}{$(2 S)(1 S)$} \\
\hline$\pi(1300) \pi$ & 8. & & 130. \\
\hline \multicolumn{4}{|c|}{$(\mathbf{1 P})(\mathbf{1 S})$} \\
\hline$a_{0}(1450) \pi$ & & 1. & \\
\hline$a_{1}(1230) \pi$ & 16. & 70. & 122. \\
\hline$a_{2}(1318) \pi$ & 43. & 86. & \\
\hline \multicolumn{4}{|c|}{$(1 S)^{2}$ strange } \\
\hline$K K$ & 20. & & 0. \\
\hline$K^{*} K$ & 17. & 33. & \\
\hline \multicolumn{4}{|c|}{ total } \\
\hline$\sum_{i} \Gamma_{i}$ & 405. & 224. & 409. \\
\hline
\end{tabular}




\begin{tabular}{|c|c|c|c|}
\hline \multicolumn{4}{|c|}{ Table B7. Partial widths of $2^{1} \mathrm{P}_{1} b_{1}$ and $h_{1}$ states $(\mathrm{MeV})$} \\
\hline Mode & $b_{1}(1700)$ & Mode & $h_{1}(1700)$ \\
\hline \multicolumn{4}{|c|}{$(1 S)^{2}$} \\
\hline$\omega \pi$ & 56. & $\rho \pi$ & 173. \\
\hline$\rho \eta$ & 18. & $\omega \eta$ & 17. \\
\hline$\rho \rho$ & 60. & & \\
\hline \multicolumn{4}{|c|}{$(2 S)(1 S)$} \\
\hline$\omega(1419) \pi$ & 13. & $\rho(1465) \pi$ & 31. \\
\hline \multicolumn{4}{|c|}{$(\mathbf{P})(\mathbf{1 S})$} \\
\hline$h_{1}(1170) \pi$ & 0. & $b_{1}(1231) \pi$ & 0. \\
\hline$a_{0}(1450) \pi$ & 2. & & \\
\hline$a_{1}(1230) \pi$ & 10. & & \\
\hline$a_{2}(1318) \pi$ & 67. & & \\
\hline \multicolumn{4}{|c|}{$(1 S)^{2}$ strange } \\
\hline$K^{*} K$ & 30. & & 30. \\
\hline \multicolumn{4}{|c|}{ total } \\
\hline$\sum_{i} \Gamma_{i}$ & 257. & & 252. \\
\hline
\end{tabular}




\begin{tabular}{|c|c|c|c|}
\hline \multicolumn{4}{|c|}{ Table B8. Partial widths of ${ }^{3} \mathrm{D}_{J} \rho_{J}$ states $(\mathrm{MeV})$. } \\
\hline Mode & $\rho_{3}(1691)$ & $\rho_{2}(1670)$ & $\rho_{1}(1700)$ \\
\hline \multicolumn{4}{|c|}{$(1 S)^{2}$} \\
\hline$\pi \pi$ & 59. & & 48. \\
\hline$\omega \pi$ & 19. & 73. & 35. \\
\hline$\rho \eta$ & 2. & 28. & 16. \\
\hline$\rho \rho$ & 71. & 15. & 14. \\
\hline \multicolumn{4}{|c|}{$(2 S)(1 S)$} \\
\hline$\pi(1300) \pi$ & 0. & & 0. \\
\hline$\omega(1419) \pi$ & 0. & 0. & 0. \\
\hline \multicolumn{4}{|c|}{$(\mathbf{P})(\mathbf{1 S})$} \\
\hline$h_{1}(1170) \pi$ & 6. & 5. & 124. \\
\hline$a_{0}(1450) \pi$ & & 0. & \\
\hline$a_{1}(1230) \pi$ & 1. & 3. & 134. \\
\hline$a_{2}(1318) \pi$ & 4. & 201. & 2. \\
\hline \multicolumn{4}{|c|}{$(1 S)^{2}$ strange } \\
\hline$K K$ & 9. & & 36. \\
\hline$K^{*} K$ & 2. & 44. & 26. \\
\hline \multicolumn{4}{|c|}{ total } \\
\hline$\sum_{i} \Gamma_{i}$ & 174. & 369. & 435. \\
\hline$\Gamma_{\text {expt }}$ & $215(20)$ & & $235(50)$ \\
\hline
\end{tabular}




\begin{tabular}{|c|c|c|c|}
\hline \multicolumn{4}{|c|}{ Table B9. Partial widths of ${ }^{3} \mathrm{D}_{J} \omega_{J}$ states $(\mathrm{MeV})$. } \\
\hline Mode & $\omega_{3}(1667)$ & $\omega_{2}(1670)$ & $\omega_{1}(1649)$ \\
\hline \multicolumn{4}{|c|}{$(\mathbf{1 S})^{2}$} \\
\hline$\rho \pi$ & 50. & 221. & 101. \\
\hline$\omega \eta$ & 2. & 27. & 13. \\
\hline \multicolumn{4}{|c|}{$(2 S)(1 S)$} \\
\hline$\rho(1465) \pi$ & 0. & 0. & 0. \\
\hline \multicolumn{4}{|c|}{$(\mathbf{P})(\mathbf{1 S})$} \\
\hline$b_{1}(1231) \pi$ & 7. & 8. & 371. \\
\hline \multicolumn{4}{|c|}{$(1 \mathrm{~S})^{2}$ strange } \\
\hline$K K$ & 8. & & 35. \\
\hline$K^{*} K$ & 2. & 44. & 21. \\
\hline \multicolumn{4}{|c|}{ total } \\
\hline$\sum_{i} \Gamma_{i}$ & 69. & 300. & 542. \\
\hline$\Gamma_{\text {expt }}$ & $168(10)$ & & $220(35)$ \\
\hline
\end{tabular}


Table B10. Partial widths of ${ }^{1} \mathrm{D}_{2} \pi_{2}$ and $\eta_{2}$ states $(\mathrm{MeV})$.

\begin{tabular}{|c|c|c|c|}
\hline Mode & $\pi_{2}(1670)$ & Mode & $\eta_{2}(1645)$ \\
\hline \multicolumn{4}{|c|}{$(1 S)^{2}$} \\
\hline$\rho \pi$ & 118. & $\rho \rho$ & 33. \\
\hline$\omega \rho$ & 41. & $\omega \omega$ & 8. \\
\hline \multicolumn{4}{|c|}{$(2 S)(1 S)$} \\
\hline$\rho(1465) \pi$ & 0. & & \\
\hline \multicolumn{4}{|c|}{$(1 \mathrm{P})(\mathbf{1 S})$} \\
\hline$b_{1}(1231) \pi$ & 0. & & \\
\hline$f_{0}(1300) \pi$ & 0. & $a_{0}(1450) \pi$ & 0. \\
\hline$f_{1}(1282) \pi$ & 1. & $a_{1}(1230) \pi$ & 5. \\
\hline$f_{2}(1275) \pi$ & 75. & $a_{2}(1318) \pi$ & 189. \\
\hline \multicolumn{4}{|c|}{$(1 \mathrm{~S})^{2}$ strange } \\
\hline$K^{*} K$ & 30. & & 26. \\
\hline \multicolumn{4}{|c|}{ total } \\
\hline$\sum_{i} \Gamma_{i}$ & 250. & & 261. \\
\hline$\Gamma_{\text {expt }}$ & $258(18)$ & & $180_{-21}^{+40}(25)$ \\
\hline
\end{tabular}




\begin{tabular}{|c|c|c|c|}
\hline \multicolumn{4}{|c|}{ Table B11. Partial widths of ${ }^{3} \mathrm{~F}_{J} a_{J}$ states $(\mathrm{MeV})$. } \\
\hline Mode & $a_{4}(2037)$ & $a_{3}(2080)$ & $a_{2}(2050)$ \\
\hline \multicolumn{4}{|c|}{$(1 \mathrm{~S})^{2}$} \\
\hline$\eta \pi$ & 12. & & 13. \\
\hline$\eta^{\prime} \pi$ & 3. & & 13. \\
\hline$\rho \pi$ & 33. & 86. & 37. \\
\hline$\omega \rho$ & 54. & 28. & 19. \\
\hline \multicolumn{4}{|c|}{$(2 S)(1 S)$} \\
\hline$\eta(1295) \pi$ & 1. & & 0. \\
\hline$\pi(1300) \eta$ & 0. & & 0. \\
\hline$\rho(1465) \pi$ & 0. & 1. & 0. \\
\hline \multicolumn{4}{|c|}{$(1 \mathrm{P})(\mathbf{1 S})$} \\
\hline$b_{1}(1231) \pi$ & 20. & 12. & 140. \\
\hline$f_{0}(1300) \pi$ & & 4. & \\
\hline$f_{1}(1282) \pi$ & 2. & 6. & 36. \\
\hline$f_{2}(1275) \pi$ & 10. & 67. & 14. \\
\hline$a_{0}(1450) \eta$ & & 0. & \\
\hline$a_{1}(1230) \eta$ & 0. & 1. & 16. \\
\hline$a_{2}(1318) \eta$ & 0. & 24. & 4. \\
\hline$h_{1}(1170) \rho$ & 0. & 40. & 21. \\
\hline$b_{1}(1231) \omega$ & 0. & 17. & 5. \\
\hline \multicolumn{4}{|c|}{$(2 P)(1 S)$} \\
\hline$b_{1}(1700) \pi$ & 0. & 0. & 2. \\
\hline$f_{0}(1700) \pi$ & & 0. & \\
\hline$f_{1}(1700) \pi$ & 0. & 0. & 0. \\
\hline$f_{2}(1700) \pi$ & 0. & 1. & 0. \\
\hline
\end{tabular}




\begin{tabular}{|c|c|c|c|}
\hline \multicolumn{4}{|c|}{ Table B11 (cont.) Partial widths of ${ }^{3} \mathrm{~F}_{J} a_{J}$ states. } \\
\hline Mode & $a_{4}(2037)$ & $a_{3}(2080)$ & $a_{2}(2050)$ \\
\hline \multicolumn{4}{|c|}{$(\mathbf{1 D})(\mathbf{1 S})$} \\
\hline$\eta_{2}(1645) \pi$ & 0. & 3. & 67. \\
\hline$\rho_{1}(1700) \pi$ & 0. & 1. & 1. \\
\hline$\rho_{2}(1670) \pi$ & 0. & 1. & 89. \\
\hline$\rho_{3}(1691) \pi$ & 2. & 127. & 1. \\
\hline \multicolumn{4}{|c|}{$(1 S)^{2}$ strange } \\
\hline$K K$ & 8. & & 14. \\
\hline$K^{*} K$ & 4. & 28. & 15. \\
\hline$K^{*} K^{*}$ & 9. & 5. & 2. \\
\hline \multicolumn{4}{|c|}{$(1 \mathrm{P})(1 \mathrm{~S})$ strange } \\
\hline$K_{0}^{*}(1429) K$ & & 0. & \\
\hline$K_{1}^{*}(1273) K$ & 0. & 3. & 91. \\
\hline$K_{1}^{*}(1402) K$ & 0. & 0. & 0. \\
\hline$K_{2}^{*}(1429) K$ & 0. & 31. & 4. \\
\hline \multicolumn{4}{|c|}{ total } \\
\hline$\sum_{i} \Gamma_{i}$ & 161. & 483. & 606. \\
\hline$\Gamma_{\text {expt }}$ & $427(120)$ & $340(80)$ & \\
\hline
\end{tabular}




\begin{tabular}{|c|c|c|c|}
\hline \multicolumn{4}{|c|}{ Table B12. Partial widths of ${ }^{3} \mathrm{~F}_{J} f_{J}$ states $(\mathrm{MeV})$} \\
\hline Mode & $f_{4}(2044)$ & $f_{3}(2050)$ & $f_{2}(2050)$ \\
\hline \multicolumn{4}{|c|}{$(1 \mathrm{~S})^{2}$} \\
\hline$\pi \pi$ & 62. & & 34. \\
\hline$\eta \eta$ & 2. & & 4. \\
\hline$\eta \eta^{\prime}$ & 0. & & 5. \\
\hline$\eta^{\prime} \eta^{\prime}$ & 0. & & 0. \\
\hline$\rho \rho$ & 86. & 37. & 31. \\
\hline$\omega \omega$ & 27. & 11. & 9. \\
\hline \multicolumn{4}{|c|}{$(2 S)(1 S)$} \\
\hline$\pi(1300) \pi$ & 2. & & 1. \\
\hline \multicolumn{4}{|c|}{$(3 S)(1 S)$} \\
\hline$\pi(1800) \pi$ & 0. & & 0. \\
\hline \multicolumn{4}{|c|}{$(1 \mathrm{P})(\mathbf{1 S})$} \\
\hline$a_{0}(1450) \pi$ & & 2. & \\
\hline$a_{1}(1230) \pi$ & 9. & 20. & 113. \\
\hline$a_{2}(1318) \pi$ & 22. & 192. & 40. \\
\hline$f_{0}(1300) \eta$ & & 0. & \\
\hline$f_{1}(1282) \eta$ & 0. & 0. & 13. \\
\hline$f_{2}(1275) \eta$ & 1. & 25. & 5. \\
\hline \multicolumn{4}{|c|}{$(2 \mathrm{P})(1 \mathrm{~S})$} \\
\hline$a_{0}(1700) \pi$ & & 0. & \\
\hline$a_{1}(1700) \pi$ & 0. & 0. & 1. \\
\hline$a_{2}(1700) \pi$ & 0. & 3. & 0. \\
\hline
\end{tabular}




\begin{tabular}{|c|c|c|c|}
\hline \multicolumn{4}{|c|}{ Table B12 (cont.) Partial widths of ${ }^{3} F_{J} f_{J}$ states. } \\
\hline Mode & $f_{4}(2044)$ & $f_{3}(2050)$ & $f_{2}(2050)$ \\
\hline \multicolumn{4}{|c|}{$(\mathbf{1 D})(1 \mathrm{~S})$} \\
\hline$\pi_{2}(1670) \pi$ & 1. & 4. & 197. \\
\hline \multicolumn{4}{|c|}{$(1 \mathrm{~S})^{2}$ strange } \\
\hline$K K$ & 9. & & 14. \\
\hline$K^{*} K$ & 5. & 26. & 15 . \\
\hline$K^{*} K^{*}$ & 10. & 4. & 2. \\
\hline \multicolumn{4}{|c|}{$(1 \mathrm{P})(\mathbf{1 S})$ strange } \\
\hline$K_{0}^{*}(1429) K$ & & 0. & \\
\hline$K_{1}^{*}(1273) K$ & 0. & 2. & 91. \\
\hline$K_{1}^{*}(1402) K$ & 0. & 0 . & 0. \\
\hline$K_{2}^{*}(1429) K$ & 0. & 23. & 4. \\
\hline \multicolumn{4}{|c|}{ total } \\
\hline$\sum_{i} \Gamma_{i}$ & 237. & 350. & 579. \\
\hline$\Gamma_{\text {expt }}$ & $208(13)$ & & \\
\hline
\end{tabular}


Table B13. Partial widths of ${ }^{1} \mathrm{~F}_{3} b_{3}$ and $h_{3}$ states $(\mathrm{MeV})$.

\begin{tabular}{||c|c||c|c||}
\hline \hline Mode & $b_{3}(2050)$ & Mode & $h_{3}(2050)$ \\
\hline \hline \multicolumn{4}{||c||}{$(15)^{2}$} \\
\hline$\rho \eta$ & 37. & $\rho \pi$ & 115. \\
\hline$\rho \eta^{\prime}$ & 13. & $\omega \eta$ & 13. \\
\hline$\rho \rho$ & 4. & $\omega \eta^{\prime}$ & 4. \\
\hline
\end{tabular}

\begin{tabular}{||l|l||c|l||}
\hline \multicolumn{4}{|c||}{$(\mathbf{2 S})(\mathbf{1 S})$} \\
\hline$\omega(1419) \pi$ & 1. & $\rho(1465) \pi$ & 1. \\
\hline$\rho(1465) \eta$ & 0. & $\omega(1419) \eta$ & 0. \\
\hline
\end{tabular}

\begin{tabular}{||l|l||l|l||}
\hline \hline \multicolumn{5}{|c||}{$(\mathbf{1 P})(\mathbf{1 S})$} \\
\hline$h_{1}(1170) \pi$ & 0. & $b_{1}(1231) \pi$ & 0. \\
\hline$b_{1}(1231) \eta$ & 0. & $h_{1}(1170) \eta$ & 0. \\
\hline$a_{0}(1450) \pi$ & 1. & & \\
\hline$a_{1}(1230) \pi$ & 14. & & \\
\hline$a_{2}(1318) \pi$ & 107. & & \\
\hline$a_{1}(1230) \omega$ & 3. & $a_{1}(1230) \rho$ & 12. \\
\hline \hline
\end{tabular}

\begin{tabular}{||c|c||c|c||}
\hline \hline \multicolumn{3}{|c||}{$(2 \mathrm{P})(1 \mathrm{~S})$} \\
\hline$h_{1}(1700) \pi$ & 0. & $b_{1}(1700) \pi$ & 0. \\
\hline$a_{0}(1700) \pi$ & 0. & & \\
\hline$a_{1}(1700) \pi$ & 0. & & \\
\hline$a_{2}(1700) \pi$ & 1. & & \\
\hline
\end{tabular}


Table B13 (cont.) Partial widths of ${ }^{1} \mathrm{~F}_{3} b_{3}$ and $h_{3}$ states $(\mathrm{MeV})$.

\begin{tabular}{|c|c|c|c|}
\hline Mode & $b_{3}(2050)$ & Mode & $h_{3}(2050)$ \\
\hline \multicolumn{4}{|c|}{$(1 \mathrm{D})(1 \mathrm{~S})$} \\
\hline$\pi_{2}(1670) \pi$ & 0. & & \\
\hline$\omega_{1}(1700) \pi$ & 0. & $\rho_{1}(1700) \pi$ & 0. \\
\hline$\omega_{2}(1670) \pi$ & 1. & $\rho_{2}(1670) \pi$ & 2. \\
\hline$\omega_{3}(1667) \pi$ & 48. & $\rho_{3}(1691) \pi$ & 138. \\
\hline \multicolumn{4}{|c|}{$(1 S)^{2}$ strange } \\
\hline$K^{*} K$ & 22. & & 22. \\
\hline$K^{*} K^{*}$ & 5. & & 5. \\
\hline \multicolumn{4}{|c|}{$(1 \mathrm{P})(1 \mathrm{~S})$ strange } \\
\hline$K_{0}^{*}(1429) K$ & 0. & & 0. \\
\hline$K_{1}^{*}(1273) K$ & 0. & & 0. \\
\hline$K_{1}^{*}(1402) K$ & 0. & & 0. \\
\hline$K_{2}^{*}(1429) K$ & 17. & & 17. \\
\hline \multicolumn{4}{|c|}{ total } \\
\hline$\sum_{i} \Gamma_{i}$ & 308. & & 330. \\
\hline
\end{tabular}

